Supporting information for:

\title{
Improving the accuracy of PCM-UAHF and PCM-UAKS calculations using optimized electrostatic scaling factors
}

Longkun Xu, Michelle L. Coote*

ARC Centre of Excellence for Electromaterials Science, Research School of Chemistry, Australian National University, Canberra, Australian Capital Territory, 2601, Australia

*Email: michelle.coote@anu.edu.au; Phone: 61261253771

\section{Table of Contents}

SECTION S1. RAW DATA OF ESF BENCHMARKING

TABLE S1. MEAN UNSIGNED ERRORS (UNIT IN KCAL/MOL) OF 1297 SOLVATION FREE ENERGIES OF NEUTRAL SOLUTES CALCULATED USING IEFPCM SOLVATION MODEL..............................................

TABLE S2. MEAN UNSIGNED ERRORS (UNIT IN KCAL/MOL) OF 1297 SOLVATION FREE ENERGIES OF NEUTRAL SOLUTES CALCULATED USING CPCM SOLVATION MODEL.................................................. 3

TABLE S3. MEAN UNSIGNED ERRORS (UNIT IN KCAL/MOL) OF 139 TRANSFER FREE ENERGIES OF NEUTRAL SOLUTES CALCULATED USING IEFPCM SOLVATION MODEL.................................................

TABLE S4. MEAN UNSIGNED ERRORS (UNIT IN KCAL/MOL) OF 139 TRANSFER FREE ENERGIES OF NEUTRAL SOLUTES CALCULATED USING CPCM SOLVATION MODEL. .................................................. 5

TABLE S5. MEAN UNSIGNED ERRORS (UNIT IN KCAL/MOL) OF 283 SOLVATION FREE ENERGIES OF IONS CALCULATED USING IEFPCM SOLVATION MODEL...............................................................

TABLE S6. MEAN UNSIGNED ERRORS (UNIT IN KCAL/MOL) OF 283 SOLVATION FREE ENERGIES OF IONS CALCULATED USING CPCM SOLVATION MODEL. ............................................................ 8

SECTION S2. RAW DATA IN PKA CALCULATIONS

11

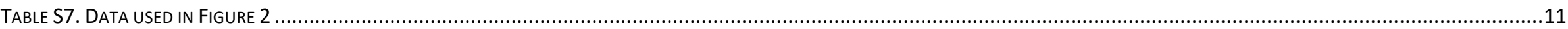

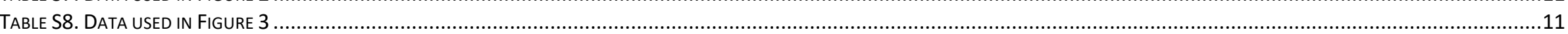

TABLE S9. DATA USED IN FIGURE 4

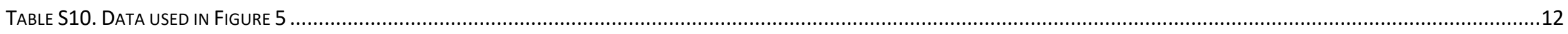

TABLE S11. OVERALL PK $K_{\text {A }}$ MAES IN ACETONITRILE OBTAINED WITH DIFFERENT ESF COMBINATIONS USING IEFPCM-UAHF, CPCM-UAHF AND CPCM-UAKS METHODS. ...................................12

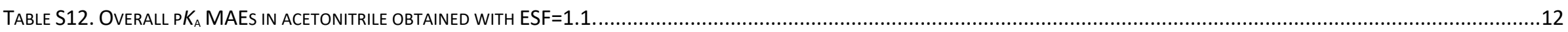




\section{Section S1. Raw data of ESF benchmarking}

Note: More detailed data can be seen in the spreadsheet of supporting information materials.

Table S1. Mean unsigned errors (unit in $\mathrm{kcal} / \mathrm{mol}$ ) of 1297 solvation free energies of neutral solutes calculated using IEFPCM solvation model.

\begin{tabular}{|c|c|c|c|c|c|c|c|c|c|c|c|c|c|c|c|}
\hline \multirow[b]{2}{*}{ Solvent } & \multirow[b]{2}{*}{$N$} & \multicolumn{7}{|c|}{ UAHF } & \multicolumn{7}{|c|}{ UAKS } \\
\hline & & $\begin{array}{l}\text { Alpha= } \\
0.9\end{array}$ & $\begin{array}{l}\text { Alpha= } \\
1.0\end{array}$ & $\begin{array}{l}\text { Alpha= } \\
1.1\end{array}$ & $\begin{array}{l}\text { Alpha= } \\
1.2\end{array}$ & $\begin{array}{l}\text { Alpha= } \\
1.3\end{array}$ & $\begin{array}{l}\text { Alpha= } \\
1.4\end{array}$ & $\begin{array}{l}\text { Alpha= } \\
1.5\end{array}$ & $\begin{array}{l}\text { Alpha= } \\
0.9\end{array}$ & $\begin{array}{l}\text { Alpha= } \\
1.0\end{array}$ & $\begin{array}{l}\text { Alpha= } \\
1.1\end{array}$ & $\begin{array}{l}\text { Alpha= } \\
1.2 \\
\end{array}$ & $\begin{array}{l}\text { Alpha= } \\
1.3\end{array}$ & $\begin{array}{l}\text { Alph= } \\
1.4\end{array}$ & $\begin{array}{l}\text { Alpha= } \\
1.5\end{array}$ \\
\hline Acetonitrile & 7 & \multirow{3}{*}{ N/A } & 7.86 & 3.31 & 2.62 & 3.78 & 4.61 & 5.16 & \multirow{2}{*}{ N/A } & 6.91 & 2.84 & 2.83 & 4.07 & 4.82 & 5.31 \\
\hline Aniline & 10 & & 7.36 & 3.20 & 4.38 & 5.97 & \multirow{4}{*}{\multicolumn{2}{|c|}{ N/A }} & & 6.73 & 2.85 & 4.72 & 6.22 & \multirow{6}{*}{\multicolumn{2}{|c|}{ N/A }} \\
\hline Benzene & 75 & & 2.47 & 2.41 & 3.54 & 4.33 & & & 12.78 & 2.34 & 2.48 & 3.66 & 4.41 & & \\
\hline Carbontet & 78 & 11.09 & 2.45 & 2.61 & 3.55 & 4.20 & & & 11.09 & 2.40 & 2.68 & 3.65 & 4.27 & & \\
\hline $\begin{array}{l}\text { Chlorobenzen } \\
\mathrm{e}\end{array}$ & 38 & \multirow{12}{*}{ N/A } & 6.90 & 1.99 & 2.12 & 2.97 & & & \multirow{12}{*}{ N/A } & 6.45 & 1.82 & 2.25 & 3.16 & & \\
\hline Chloroform & 108 & & 6.27 & 2.31 & 2.84 & 3.85 & & & & 5.96 & 2.30 & 2.93 & 3.96 & & \\
\hline Cyclohexane & 91 & & 2.90 & 1.44 & 1.75 & 2.11 & & & & 2.73 & 1.40 & 1.77 & 2.12 & & \\
\hline $\begin{array}{l}\text { Dichloroethan } \\
\text { e }\end{array}$ & 39 & & 11.10 & 3.04 & 1.38 & 2.54 & 3.53 & 4.24 & & 10.25 & 2.61 & 1.56 & 2.77 & 3.72 & 4.38 \\
\hline Diethylether & 70 & & 6.78 & 2.06 & 2.40 & 3.38 & \multicolumn{2}{|c|}{$\mathrm{N} / \mathrm{A}$} & & 6.40 & 1.99 & 2.50 & 3.56 & \multicolumn{2}{|c|}{$\mathrm{N} / \mathrm{A}$} \\
\hline $\begin{array}{l}\text { Dimethylsulfo } \\
\text { xide }\end{array}$ & 7 & & 8.72 & 3.91 & 2.46 & 2.35 & 2.82 & 3.27 & & 8.03 & 3.47 & 2.16 & 2.54 & 3.01 & 3.50 \\
\hline Ethanol & 8 & & 11.74 & 5.23 & 2.77 & 1.72 & 1.57 & 1.64 & & 10.65 & 4.44 & 2.22 & 1.51 & 1.53 & 1.69 \\
\hline Heptane & 68 & & 2.86 & 1.29 & 1.57 & 2.05 & \multicolumn{2}{|c|}{$\mathrm{N} / \mathrm{A}$} & & 2.76 & 1.21 & 1.60 & 2.08 & \multicolumn{2}{|c|}{ N/A } \\
\hline $\begin{array}{l}\text { Methylenechl } \\
\text { oride }\end{array}$ & 11 & & 9.70 & 3.80 & 2.02 & 2.34 & 2.85 & 3.37 & & 8.89 & 3.26 & 1.95 & 2.44 & 3.05 & 3.56 \\
\hline Nitromethane & 7 & & 11.37 & 4.76 & 2.48 & 1.86 & 2.25 & 2.69 & & 10.18 & 3.95 & 2.03 & 1.94 & 2.43 & 2.96 \\
\hline Octanol & 241 & & 6.64 & 3.33 & 5.46 & 7.04 & \multicolumn{2}{|c|}{$\mathrm{N} / \mathrm{A}$} & & 6.38 & 3.53 & 5.70 & 7.22 & \multicolumn{2}{|c|}{$\mathrm{N} / \mathrm{A}$} \\
\hline $\begin{array}{l}\text { Tetrahydrofur } \\
\text { an }\end{array}$ & 7 & & 7.81 & 3.48 & 1.98 & 2.16 & 2.70 & 3.14 & & 7.02 & 2.93 & 1.81 & 2.35 & 2.94 & 3.32 \\
\hline
\end{tabular}




\begin{tabular}{|c|c|c|c|c|c|c|c|c|c|c|c|c|c|}
\hline Toluene & 51 & 3.56 & 1.55 & 2.19 & 2.88 & \multicolumn{2}{|c|}{$\mathrm{N} / \mathrm{A}$} & 3.22 & 1.57 & 2.30 & 2.99 & \multicolumn{2}{|c|}{$\mathrm{N} / \mathrm{A}$} \\
\hline Water & 381 & 14.37 & 4.19 & 1.39 & 2.68 & 3.90 & 4.72 & 13.86 & 3.81 & 1.36 & 2.85 & 4.03 & 4.79 \\
\hline All solvents & $\begin{array}{c}129 \\
7\end{array}$ & 8.07 & 3.00 & 2.72 & 3.76 & \multicolumn{2}{|c|}{ N/A } & 7.70 & 2.88 & 2.79 & 3.90 & \multicolumn{2}{|c|}{ N/A } \\
\hline
\end{tabular}

Table S2. Mean unsigned errors (unit in $\mathrm{kcal} / \mathrm{mol}$ ) of 1297 solvation free energies of neutral solutes calculated using CPCM solvation model.

\begin{tabular}{|c|c|c|c|c|c|c|c|c|c|c|c|c|c|}
\hline \multirow[b]{2}{*}{ Solvent } & \multirow[b]{2}{*}{$N$} & \multicolumn{6}{|c|}{ UAHF } & \multicolumn{6}{|c|}{ UAKS } \\
\hline & & $\begin{array}{l}\text { Alpha=1. } \\
0\end{array}$ & $\begin{array}{l}\text { Alpha=1. } \\
1\end{array}$ & $\begin{array}{l}\text { Alpha=1. } \\
2\end{array}$ & $\begin{array}{l}\text { Alpha=1. } \\
3\end{array}$ & $\begin{array}{l}\text { Alpha=1. } \\
4\end{array}$ & $\begin{array}{l}\text { Alpha=1. } \\
5\end{array}$ & $\begin{array}{l}\text { Alpha=1. } \\
0\end{array}$ & $\begin{array}{l}\text { Alpha=1. } \\
1\end{array}$ & $\begin{array}{l}\text { Alpha=1. } \\
2\end{array}$ & $\begin{array}{l}\text { Alpha=1. } \\
3\end{array}$ & $\begin{array}{l}\text { Alph=1. } \\
4\end{array}$ & $\begin{array}{l}\text { Alpha=1. } \\
5\end{array}$ \\
\hline Acetonitrile & 7 & 8.09 & 3.37 & 2.59 & 3.72 & 4.57 & 5.13 & 7.13 & 2.87 & 2.76 & 4.03 & 4.79 & 5.29 \\
\hline Aniline & 10 & 8.57 & 3.55 & 4.05 & 5.66 & \multirow{3}{*}{\multicolumn{2}{|c|}{$\mathrm{N} / \mathrm{A}$}} & 7.90 & 3.05 & 4.29 & 5.93 & \multirow{3}{*}{\multicolumn{2}{|c|}{ N/A }} \\
\hline Benzene & 75 & 4.42 & 1.99 & 2.95 & 3.86 & & & 4.17 & 2.00 & 3.07 & 3.97 & & \\
\hline Carbontet & 78 & 3.67 & 2.23 & 3.04 & 3.80 & & & 3.50 & 2.23 & 3.14 & 3.90 & & \\
\hline Chlorobenzene & 38 & 8.41 & 2.41 & 1.91 & 2.69 & 3.46 & 4.00 & 7.93 & 2.25 & 2.00 & 2.89 & 3.63 & 4.12 \\
\hline Chloroform & 108 & 8.09 & $\begin{array}{c}2.54 \\
(2.539)\end{array}$ & $\begin{array}{c}2.54 \\
(2.543)\end{array}$ & 3.51 & \multicolumn{2}{|c|}{ N/A } & 7.76 & 2.51 & 2.59 & 3.64 & \multicolumn{2}{|c|}{$\mathrm{N} / \mathrm{A}$} \\
\hline Cyclohexane & 91 & 4.65 & 1.71 & 1.50 & 1.84 & 2.15 & 2.38 & 4.42 & 1.64 & 1.51 & 1.86 & 2.16 & 2.38 \\
\hline Dichloroethane & 39 & 12.31 & 3.64 & 1.30 & 2.31 & 3.36 & 4.11 & 11.42 & 3.10 & 1.41 & 2.57 & 3.55 & 4.25 \\
\hline Diethylether & 70 & 8.85 & 2.65 & 2.11 & 2.98 & 3.84 & 4.42 & 8.44 & 2.45 & 2.17 & 3.18 & 4.00 & 4.54 \\
\hline $\begin{array}{l}\text { Dimethylsulfoxid } \\
\text { e }\end{array}$ & 7 & 8.90 & 3.96 & 2.49 & 2.33 & 2.81 & 3.25 & 8.18 & 3.51 & 2.18 & 2.52 & 2.99 & 3.48 \\
\hline Ethanol & 8 & 12.11 & 5.42 & 2.86 & 1.74 & 1.57 & 1.63 & 11.04 & 4.61 & 2.30 & 1.51 & 1.52 & 1.67 \\
\hline Heptane & 68 & 4.58 & 1.65 & 1.30 & 1.68 & 2.10 & 2.40 & 4.45 & 1.61 & 1.29 & 1.73 & 2.13 & 2.40 \\
\hline $\begin{array}{l}\text { Methylenechlori } \\
\text { de }\end{array}$ & 11 & 10.88 & 4.26 & 2.11 & 2.22 & 2.71 & 3.23 & 10.02 & 3.69 & 1.88 & 2.31 & 2.92 & 3.43 \\
\hline Nitromethane & 7 & 11.64 & 4.87 & 2.52 & 1.85 & 2.22 & 2.66 & 10.43 & 4.04 & 2.07 & 1.91 & 2.40 & 2.94 \\
\hline Octanol & 241 & 7.60 & 3.20 & 5.16 & 6.81 & \multicolumn{2}{|c|}{$\mathrm{N} / \mathrm{A}$} & 7.29 & 3.36 & 5.39 & 7.01 & \multicolumn{2}{|c|}{ N/A } \\
\hline Tetrahydrofuran & 7 & 8.76 & 3.84 & 2.12 & 2.04 & 2.55 & 3.02 & 7.93 & 3.25 & 1.81 & 2.19 & 2.81 & 3.22 \\
\hline Toluene & 51 & 5.69 & 1.64 & 1.78 & 2.47 & \multicolumn{2}{|c|}{ N/A } & 5.27 & 1.50 & 1.87 & 2.60 & \multicolumn{2}{|c|}{$\mathrm{N} / \mathrm{A}$} \\
\hline Water & 381 & 14.57 & 4.29 & 1.38 & 2.64 & 3.87 & 4.70 & 14.06 & 3.90 & 1.35 & 2.81 & 4.01 & 4.77 \\
\hline All solvents & $\begin{array}{c}129 \\
7\end{array}$ & 9.16 & 3.09 & 2.50 & 3.54 & \multicolumn{2}{|c|}{$\mathrm{N} / \mathrm{A}$} & 8.76 & 2.94 & 2.56 & 3.68 & \multicolumn{2}{|c|}{$\mathrm{N} / \mathrm{A}$} \\
\hline
\end{tabular}


Table S3. Mean unsigned errors (unit in $\mathrm{kcal} / \mathrm{mol}$ ) of 139 transfer free energies of neutral solutes calculated using IEFPCM solvation model.

\begin{tabular}{|c|c|c|c|c|c|c|c|c|c|c|c|c|c|c|c|c|c|c|c|c|c|c|c|c|c|}
\hline \multirow{2}{*}{$\begin{array}{l}\text { Solvent- } \\
\text { water }\end{array}$} & \multirow[t]{2}{*}{$\mathrm{N}$} & \multicolumn{12}{|c|}{ UAHF } & \multicolumn{12}{|c|}{ UAKS } \\
\hline & & $\begin{array}{l}\text { Alpha= } \\
1.0\end{array}$ & 1.1 & 1.2 & 1.3 & 1.4 & 1.5 & 1.6 & 1.7 & 1.8 & 1.9 & 2.0 & 2.1 & $\begin{array}{l}\text { Alpha= } \\
1.0\end{array}$ & 1.1 & 1.2 & 1.3 & 1.4 & 1.5 & 1.6 & 1.7 & 1.8 & 1.9 & 2.0 & 2.1 \\
\hline Benzene & 4 & 13.25 & $\begin{array}{l}6.9 \\
7\end{array}$ & $\begin{array}{l}4.0 \\
7\end{array}$ & $\begin{array}{l}2.4 \\
5\end{array}$ & $\begin{array}{l}1.4 \\
5\end{array}$ & $\begin{array}{l}0.9 \\
4\end{array}$ & $\begin{array}{l}0.7 \\
4\end{array}$ & $\begin{array}{l}0.7 \\
4\end{array}$ & $\begin{array}{l}0.7 \\
8\end{array}$ & $\begin{array}{l}0.8 \\
9\end{array}$ & $\begin{array}{l}0.9 \\
7\end{array}$ & $\begin{array}{l}1.0 \\
3\end{array}$ & 14.29 & $\begin{array}{l}7.2 \\
4\end{array}$ & $\begin{array}{l}4.1 \\
0\end{array}$ & $\begin{array}{l}2.4 \\
1\end{array}$ & $\begin{array}{l}1.4 \\
1\end{array}$ & $\begin{array}{l}0.9 \\
2\end{array}$ & $\begin{array}{l}0.6 \\
0\end{array}$ & $\begin{array}{l}0.6 \\
2\end{array}$ & $\begin{array}{l}0.6 \\
8\end{array}$ & $\begin{array}{l}0.7 \\
9\end{array}$ & $\begin{array}{l}0.8 \\
8\end{array}$ & $\begin{array}{l}0.9 \\
5\end{array}$ \\
\hline Carbontet & 2 & 10.57 & $\begin{array}{l}5.5 \\
5\end{array}$ & $\begin{array}{l}3.3 \\
7\end{array}$ & $\begin{array}{l}2.2 \\
0\end{array}$ & $\begin{array}{l}1.4 \\
8\end{array}$ & $\begin{array}{l}1.2 \\
3\end{array}$ & $\begin{array}{l}1.1 \\
5\end{array}$ & $\begin{array}{l}1.0 \\
8\end{array}$ & $\begin{array}{l}1.0 \\
3\end{array}$ & $\begin{array}{l}0.9 \\
8\end{array}$ & $\begin{array}{l}0.9 \\
5\end{array}$ & $\begin{array}{l}0.9 \\
1\end{array}$ & 9.49 & $\begin{array}{l}4.8 \\
2\end{array}$ & $\begin{array}{l}2.8 \\
5\end{array}$ & $\begin{array}{l}1.8 \\
2\end{array}$ & $\begin{array}{l}1.1 \\
9\end{array}$ & $\begin{array}{l}1.0 \\
2\end{array}$ & $\begin{array}{l}0.9 \\
7\end{array}$ & $\begin{array}{l}0.9 \\
3\end{array}$ & $\begin{array}{l}0.8 \\
9\end{array}$ & $\begin{array}{l}0.8 \\
6\end{array}$ & $\begin{array}{l}0.8 \\
4\end{array}$ & $\begin{array}{l}0.8 \\
2\end{array}$ \\
\hline $\begin{array}{l}\text { Chloroben } \\
\text { zene }\end{array}$ & 1 & 3.32 & $\begin{array}{l}1.3 \\
9\end{array}$ & $\begin{array}{l}0.5 \\
8\end{array}$ & $\begin{array}{l}0.1 \\
6\end{array}$ & $\begin{array}{l}0.1 \\
0\end{array}$ & $\begin{array}{l}0.2 \\
6\end{array}$ & $\begin{array}{l}0.3 \\
7\end{array}$ & $\begin{array}{l}0.4 \\
6\end{array}$ & $\begin{array}{l}0.5 \\
2\end{array}$ & $\begin{array}{l}0.5 \\
7\end{array}$ & $\begin{array}{l}0.6 \\
1\end{array}$ & $\begin{array}{l}0.6 \\
4\end{array}$ & 3.11 & $\begin{array}{l}1.3 \\
0\end{array}$ & $\begin{array}{l}0.5 \\
4\end{array}$ & $\begin{array}{l}0.1 \\
6\end{array}$ & $\begin{array}{l}0.0 \\
8\end{array}$ & $\begin{array}{l}0.2 \\
3\end{array}$ & $\begin{array}{l}0.3 \\
4\end{array}$ & $\begin{array}{l}0.4 \\
2\end{array}$ & $\begin{array}{l}0.4 \\
8\end{array}$ & $\begin{array}{l}0.5 \\
2\end{array}$ & $\begin{array}{l}0.5 \\
6\end{array}$ & $\begin{array}{l}0.5 \\
9\end{array}$ \\
\hline $\begin{array}{l}\text { Chlorofor } \\
\text { m }\end{array}$ & 7 & 7.40 & $\begin{array}{l}3.7 \\
5\end{array}$ & $\begin{array}{l}2.1 \\
0\end{array}$ & $\begin{array}{l}1.2 \\
7\end{array}$ & $\begin{array}{l}0.8 \\
9\end{array}$ & $\begin{array}{l}0.8 \\
1\end{array}$ & $\begin{array}{l}0.8 \\
7\end{array}$ & $\begin{array}{l}0.9 \\
2\end{array}$ & $\begin{array}{l}0.9 \\
5\end{array}$ & $\begin{array}{l}0.9 \\
8\end{array}$ & $\begin{array}{l}1.0 \\
0\end{array}$ & $\begin{array}{l}1.0 \\
1\end{array}$ & 7.38 & $\begin{array}{l}3.6 \\
6\end{array}$ & $\begin{array}{l}2.0 \\
2\end{array}$ & $\begin{array}{l}1.2 \\
4\end{array}$ & $\begin{array}{l}0.8 \\
8\end{array}$ & $\begin{array}{l}0.8 \\
1\end{array}$ & $\begin{array}{l}0.8 \\
7\end{array}$ & $\begin{array}{l}0.9 \\
2\end{array}$ & $\begin{array}{l}0.9 \\
5\end{array}$ & $\begin{array}{l}0.9 \\
7\end{array}$ & $\begin{array}{l}0.9 \\
9\end{array}$ & $\begin{array}{l}1.0 \\
1\end{array}$ \\
\hline $\begin{array}{l}\text { Cyclohexa } \\
\text { ne }\end{array}$ & 5 & 9.72 & $\begin{array}{l}3.1 \\
8\end{array}$ & $\begin{array}{l}1.1 \\
8\end{array}$ & $\begin{array}{l}1.6 \\
2 \\
\end{array}$ & $\begin{array}{l}2.2 \\
7 \\
\end{array}$ & $\begin{array}{l}2.8 \\
2 \\
\end{array}$ & $\begin{array}{l}3.2 \\
6 \\
\end{array}$ & $\begin{array}{l}3.5 \\
9 \\
\end{array}$ & $\begin{array}{l}3.8 \\
5 \\
\end{array}$ & $\begin{array}{l}4.0 \\
5 \\
\end{array}$ & $\begin{array}{l}4.2 \\
0 \\
\end{array}$ & $\begin{array}{l}4.3 \\
3 \\
\end{array}$ & 9.41 & $\begin{array}{l}2.8 \\
5 \\
\end{array}$ & $\begin{array}{l}1.1 \\
4\end{array}$ & $\begin{array}{l}1.7 \\
7 \\
\end{array}$ & $\begin{array}{l}2.3 \\
9 \\
\end{array}$ & $\begin{array}{l}2.9 \\
6 \\
\end{array}$ & $\begin{array}{l}3.3 \\
8\end{array}$ & $\begin{array}{l}3.6 \\
9 \\
\end{array}$ & $\begin{array}{l}3.9 \\
2 \\
\end{array}$ & $\begin{array}{l}4.1 \\
0 \\
\end{array}$ & $\begin{array}{l}4.2 \\
5 \\
\end{array}$ & $\begin{array}{l}4.3 \\
7 \\
\end{array}$ \\
\hline $\begin{array}{l}\text { Dichloroet } \\
\text { hane }\end{array}$ & 3 & 2.45 & $\begin{array}{l}0.6 \\
0\end{array}$ & $\begin{array}{l}0.5 \\
0\end{array}$ & $\begin{array}{l}0.7 \\
3\end{array}$ & $\begin{array}{l}0.8 \\
6\end{array}$ & $\begin{array}{l}0.9 \\
5\end{array}$ & $\begin{array}{l}1.0 \\
2\end{array}$ & $\begin{array}{l}1.0 \\
9\end{array}$ & $\begin{array}{l}1.1 \\
4\end{array}$ & $\begin{array}{l}1.1 \\
8\end{array}$ & $\begin{array}{l}1.2 \\
1\end{array}$ & $\begin{array}{l}1.2 \\
4\end{array}$ & 2.15 & $\begin{array}{l}0.4 \\
3\end{array}$ & $\begin{array}{l}0.6 \\
2\end{array}$ & $\begin{array}{l}0.8 \\
2\end{array}$ & $\begin{array}{l}0.9 \\
3\end{array}$ & $\begin{array}{l}0.9 \\
9\end{array}$ & $\begin{array}{l}1.0 \\
4\end{array}$ & $\begin{array}{l}1.0 \\
8\end{array}$ & $\begin{array}{l}1.1 \\
2\end{array}$ & $\begin{array}{l}1.1 \\
6\end{array}$ & $\begin{array}{l}1.1 \\
8\end{array}$ & $\begin{array}{l}1.2 \\
0\end{array}$ \\
\hline $\begin{array}{l}\text { Diethyleth } \\
\text { er }\end{array}$ & 8 & 9.53 & $\begin{array}{l}4.5 \\
2\end{array}$ & $\begin{array}{l}2.2 \\
1\end{array}$ & $\begin{array}{l}1.4 \\
7\end{array}$ & $\begin{array}{l}1.2 \\
9\end{array}$ & $\begin{array}{l}1.2 \\
9\end{array}$ & $\begin{array}{l}1.3 \\
1\end{array}$ & $\begin{array}{l}1.3 \\
3\end{array}$ & $\begin{array}{l}1.3 \\
8\end{array}$ & $\begin{array}{l}1.4 \\
3\end{array}$ & $\begin{array}{l}1.4 \\
7\end{array}$ & $\begin{array}{l}1.5 \\
3\end{array}$ & 9.88 & $\begin{array}{l}4.5 \\
9\end{array}$ & $\begin{array}{l}2.2 \\
2\end{array}$ & $\begin{array}{l}1.4 \\
8\end{array}$ & $\begin{array}{l}1.3 \\
1\end{array}$ & $\begin{array}{l}1.2 \\
7\end{array}$ & $\begin{array}{l}1.2 \\
9\end{array}$ & $\begin{array}{l}1.3 \\
1\end{array}$ & $\begin{array}{l}1.3 \\
5\end{array}$ & $\begin{array}{l}1.4 \\
1\end{array}$ & $\begin{array}{l}1.4 \\
5\end{array}$ & $\begin{array}{l}1.4 \\
9\end{array}$ \\
\hline Heptane & 6 & 11.01 & $\begin{array}{l}4.1 \\
0\end{array}$ & $\begin{array}{l}1.7 \\
0\end{array}$ & $\begin{array}{l}1.8 \\
2\end{array}$ & $\begin{array}{l}2.5 \\
0\end{array}$ & $\begin{array}{l}3.2 \\
1\end{array}$ & $\begin{array}{l}3.7 \\
0\end{array}$ & $\begin{array}{l}4.0 \\
5\end{array}$ & $\begin{array}{l}4.3 \\
2\end{array}$ & $\begin{array}{l}4.5 \\
3\end{array}$ & $\begin{array}{l}4.7 \\
0\end{array}$ & $\begin{array}{l}4.8 \\
3\end{array}$ & 11.29 & $\begin{array}{l}4.2 \\
4\end{array}$ & $\begin{array}{l}1.8 \\
6\end{array}$ & $\begin{array}{l}1.9 \\
3\end{array}$ & $\begin{array}{l}2.5 \\
4\end{array}$ & $\begin{array}{l}3.2 \\
4\end{array}$ & $\begin{array}{l}3.7 \\
2\end{array}$ & $\begin{array}{l}4.0 \\
7\end{array}$ & $\begin{array}{l}4.3 \\
3\end{array}$ & $\begin{array}{l}4.5 \\
3\end{array}$ & $\begin{array}{l}4.6 \\
9\end{array}$ & $\begin{array}{l}4.8 \\
2\end{array}$ \\
\hline Octanol & $\begin{array}{l}10 \\
3\end{array}$ & 8.71 & $\begin{array}{l}6.9 \\
5\end{array}$ & $\begin{array}{l}6.1 \\
8\end{array}$ & $\begin{array}{l}5.7 \\
8\end{array}$ & $\begin{array}{l}5.5 \\
4\end{array}$ & $\begin{array}{l}5.3 \\
9\end{array}$ & $\begin{array}{l}5.2 \\
8\end{array}$ & $\begin{array}{l}5.2 \\
1\end{array}$ & $\begin{array}{l}5.1 \\
5\end{array}$ & $\begin{array}{l}5.1 \\
1\end{array}$ & $\begin{array}{l}5.0 \\
7\end{array}$ & $\begin{array}{l}5.0 \\
5\end{array}$ & 8.77 & $\begin{array}{l}6.9 \\
3\end{array}$ & $\begin{array}{l}6.1 \\
5\end{array}$ & $\begin{array}{l}5.7 \\
5\end{array}$ & $\begin{array}{l}5.5 \\
2\end{array}$ & $\begin{array}{l}5.3 \\
7\end{array}$ & $\begin{array}{l}5.2 \\
6\end{array}$ & $\begin{array}{l}5.1 \\
9\end{array}$ & $\begin{array}{l}5.1 \\
4\end{array}$ & $\begin{array}{l}5.1 \\
0\end{array}$ & $\begin{array}{l}5.0 \\
6\end{array}$ & $\begin{array}{l}5.0 \\
4\end{array}$ \\
\hline All & $\begin{array}{l}13 \\
9\end{array}$ & 8.81 & $\begin{array}{l}6.1 \\
9\end{array}$ & $\begin{array}{l}5.1 \\
1\end{array}$ & $\begin{array}{l}4.6 \\
9\end{array}$ & $\begin{array}{l}4.5 \\
0\end{array}$ & $\begin{array}{l}4.4 \\
2\end{array}$ & $\begin{array}{l}4.3 \\
7\end{array}$ & $\begin{array}{l}4.3 \\
5\end{array}$ & $\begin{array}{l}4.3 \\
3\end{array}$ & $\begin{array}{l}4.3 \\
3\end{array}$ & $\begin{array}{l}4.3 \\
2\end{array}$ & $\begin{array}{l}4.3 \\
2\end{array}$ & 8.88 & $\begin{array}{l}6.1 \\
7\end{array}$ & $\begin{array}{l}5.0 \\
9\end{array}$ & $\begin{array}{l}4.6 \\
7\end{array}$ & $\begin{array}{l}4.4 \\
8\end{array}$ & $\begin{array}{l}4.4 \\
0\end{array}$ & $\begin{array}{l}4.3 \\
6\end{array}$ & $\begin{array}{l}4.3 \\
3\end{array}$ & $\begin{array}{l}4.3 \\
2\end{array}$ & $\begin{array}{l}4.3 \\
1\end{array}$ & $\begin{array}{l}4.3 \\
1\end{array}$ & $\begin{array}{l}4.3 \\
0\end{array}$ \\
\hline
\end{tabular}


Table S4. Mean unsigned errors (unit in $\mathrm{kcal} / \mathrm{mol}$ ) of 139 transfer free energies of neutral solutes calculated using CPCM solvation model.

\begin{tabular}{|c|c|c|c|c|c|c|c|c|c|c|c|c|c|c|c|c|c|c|c|c|c|c|c|c|c|}
\hline \multirow{2}{*}{$\begin{array}{l}\text { Solvent- } \\
\text { water }\end{array}$} & \multirow[t]{2}{*}{$\mathrm{N}$} & \multicolumn{12}{|c|}{ UAHF } & \multicolumn{12}{|c|}{ UAKS } \\
\hline & & $\begin{array}{l}\text { Alpha= } \\
1.0\end{array}$ & 1.1 & 1.2 & 1.3 & 1.4 & 1.5 & 1.6 & 1.7 & 1.8 & 1.9 & 2.0 & 2.1 & $\begin{array}{l}\text { Alpha= } \\
1.0\end{array}$ & 1.1 & 1.2 & 1.3 & 1.4 & 1.5 & 1.6 & 1.7 & 1.8 & 1.9 & 2.0 & 2.1 \\
\hline Benzene & 4 & 10.84 & $\begin{array}{l}5.6 \\
1\end{array}$ & $\begin{array}{l}3.1 \\
6\end{array}$ & $\begin{array}{l}1.7 \\
9\end{array}$ & $\begin{array}{l}1.0 \\
6\end{array}$ & $\begin{array}{l}0.7 \\
0\end{array}$ & $\begin{array}{l}0.7 \\
2\end{array}$ & $\begin{array}{l}0.7 \\
8\end{array}$ & $\begin{array}{l}0.9 \\
0\end{array}$ & $\begin{array}{l}0.9 \\
8\end{array}$ & $\begin{array}{l}1.0 \\
5\end{array}$ & $\begin{array}{l}1.1 \\
0\end{array}$ & 11.66 & $\begin{array}{l}5.8 \\
3\end{array}$ & $\begin{array}{l}3.2 \\
0\end{array}$ & $\begin{array}{l}1.7 \\
7\end{array}$ & $\begin{array}{l}1.0 \\
5\end{array}$ & $\begin{array}{l}0.6 \\
4\end{array}$ & $\begin{array}{l}0.6 \\
0 \\
\end{array}$ & $\begin{array}{l}0.6 \\
7\end{array}$ & $\begin{array}{l}0.8 \\
0\end{array}$ & $\begin{array}{l}0.8 \\
9\end{array}$ & $\begin{array}{l}0.9 \\
6 \\
\end{array}$ & $\begin{array}{l}1.0 \\
1 \\
\end{array}$ \\
\hline Carbontet & 2 & 8.83 & $\begin{array}{l}4.6 \\
3\end{array}$ & $\begin{array}{l}2.7 \\
7\end{array}$ & $\begin{array}{l}1.7 \\
6\end{array}$ & $\begin{array}{l}1.2 \\
6\end{array}$ & $\begin{array}{l}1.1 \\
6\end{array}$ & $\begin{array}{l}1.0 \\
9\end{array}$ & $\begin{array}{l}1.0 \\
3\end{array}$ & $\begin{array}{l}0.9 \\
8\end{array}$ & $\begin{array}{l}0.9 \\
4\end{array}$ & $\begin{array}{l}0.9 \\
1\end{array}$ & $\begin{array}{l}0.8 \\
8\end{array}$ & 7.91 & $\begin{array}{l}4.0 \\
1\end{array}$ & $\begin{array}{l}2.3 \\
3\end{array}$ & $\begin{array}{l}1.4 \\
4\end{array}$ & $\begin{array}{l}1.0 \\
4\end{array}$ & $\begin{array}{l}0.9 \\
8\end{array}$ & $\begin{array}{l}0.9 \\
3\end{array}$ & $\begin{array}{l}0.9 \\
0\end{array}$ & $\begin{array}{l}0.8 \\
7\end{array}$ & $\begin{array}{l}0.8 \\
4\end{array}$ & $\begin{array}{l}0.8 \\
2\end{array}$ & $\begin{array}{l}0.8 \\
0\end{array}$ \\
\hline $\begin{array}{l}\text { Chloroben } \\
\text { zene }\end{array}$ & 1 & 2.25 & $\begin{array}{l}0.8 \\
5 \\
\end{array}$ & $\begin{array}{l}0.2 \\
4 \\
\end{array}$ & $\begin{array}{l}0.0 \\
9 \\
\end{array}$ & $\begin{array}{l}0.2 \\
8 \\
\end{array}$ & $\begin{array}{l}0.4 \\
0 \\
\end{array}$ & $\begin{array}{l}0.4 \\
9 \\
\end{array}$ & $\begin{array}{l}0.5 \\
6 \\
\end{array}$ & $\begin{array}{l}0.6 \\
1 \\
\end{array}$ & $\begin{array}{l}0.6 \\
4 \\
\end{array}$ & $\begin{array}{l}0.6 \\
7 \\
\end{array}$ & $\begin{array}{l}0.7 \\
0 \\
\end{array}$ & 2.12 & $\begin{array}{l}0.8 \\
0 \\
\end{array}$ & $\begin{array}{l}0.2 \\
3 \\
\end{array}$ & $\begin{array}{l}0.0 \\
7 \\
\end{array}$ & $\begin{array}{l}0.2 \\
5 \\
\end{array}$ & $\begin{array}{l}0.3 \\
7 \\
\end{array}$ & $\begin{array}{l}0.4 \\
5 \\
\end{array}$ & $\begin{array}{l}0.5 \\
1 \\
\end{array}$ & $\begin{array}{l}0.5 \\
6 \\
\end{array}$ & $\begin{array}{l}0.5 \\
9 \\
\end{array}$ & $\begin{array}{l}0.6 \\
2 \\
\end{array}$ & $\begin{array}{l}0.6 \\
4 \\
\end{array}$ \\
\hline $\begin{array}{l}\text { Chlorofor } \\
\mathrm{m}\end{array}$ & 7 & 5.25 & $\begin{array}{l}2.5 \\
6 \\
\end{array}$ & $\begin{array}{l}1.3 \\
3 \\
\end{array}$ & $\begin{array}{l}0.8 \\
7 \\
\end{array}$ & $\begin{array}{l}0.8 \\
0 \\
\end{array}$ & $\begin{array}{l}0.8 \\
8 \\
\end{array}$ & $\begin{array}{l}0.9 \\
3 \\
\end{array}$ & $\begin{array}{l}0.9 \\
6 \\
\end{array}$ & $\begin{array}{l}0.9 \\
9 \\
\end{array}$ & $\begin{array}{l}1.0 \\
1 \\
\end{array}$ & $\begin{array}{l}1.0 \\
2 \\
\end{array}$ & $\begin{array}{l}1.0 \\
3 \\
\end{array}$ & 5.21 & $\begin{array}{l}2.5 \\
0 \\
\end{array}$ & $\begin{array}{l}1.3 \\
0 \\
\end{array}$ & $\begin{array}{l}0.8 \\
5 \\
\end{array}$ & $\begin{array}{l}0.8 \\
0 \\
\end{array}$ & $\begin{array}{l}0.8 \\
8 \\
\end{array}$ & $\begin{array}{l}0.9 \\
2 \\
\end{array}$ & $\begin{array}{l}0.9 \\
6 \\
\end{array}$ & $\begin{array}{l}0.9 \\
8 \\
\end{array}$ & $\begin{array}{l}1.0 \\
0 \\
\end{array}$ & $\begin{array}{l}1.0 \\
1 \\
\end{array}$ & $\begin{array}{l}1.0 \\
3 \\
\end{array}$ \\
\hline $\begin{array}{l}\text { Cyclohexa } \\
\text { ne }\end{array}$ & 5 & 7.46 & $\begin{array}{l}1.9 \\
4\end{array}$ & $\begin{array}{l}1.1 \\
3\end{array}$ & $\begin{array}{l}2.0 \\
0\end{array}$ & $\begin{array}{l}2.6 \\
3\end{array}$ & $\begin{array}{l}3.1 \\
7\end{array}$ & $\begin{array}{l}3.5 \\
5\end{array}$ & $\begin{array}{l}3.8 \\
4\end{array}$ & $\begin{array}{l}4.0 \\
5\end{array}$ & $\begin{array}{l}4.2 \\
2\end{array}$ & $\begin{array}{l}4.3 \\
5\end{array}$ & $\begin{array}{l}4.4 \\
6\end{array}$ & 7.17 & $\begin{array}{l}1.6 \\
6\end{array}$ & $\begin{array}{l}1.2 \\
4\end{array}$ & $\begin{array}{l}2.1 \\
3\end{array}$ & $\begin{array}{l}2.7 \\
8\end{array}$ & $\begin{array}{l}3.2 \\
9\end{array}$ & $\begin{array}{l}3.6 \\
4\end{array}$ & $\begin{array}{l}3.9 \\
1\end{array}$ & $\begin{array}{l}4.1 \\
1\end{array}$ & $\begin{array}{l}4.2 \\
6\end{array}$ & $\begin{array}{l}4.3 \\
9\end{array}$ & $\begin{array}{l}4.4 \\
9\end{array}$ \\
\hline $\begin{array}{l}\text { Dichloroet } \\
\text { hane }\end{array}$ & 3 & 1.29 & $\begin{array}{l}0.4 \\
7 \\
\end{array}$ & $\begin{array}{l}0.7 \\
2 \\
\end{array}$ & $\begin{array}{l}0.8 \\
8 \\
\end{array}$ & $\begin{array}{l}0.9 \\
7 \\
\end{array}$ & $\begin{array}{l}1.0 \\
5 \\
\end{array}$ & $\begin{array}{l}1.1 \\
2 \\
\end{array}$ & $\begin{array}{l}1.1 \\
7 \\
\end{array}$ & $\begin{array}{l}1.2 \\
1 \\
\end{array}$ & $\begin{array}{l}1.2 \\
4 \\
\end{array}$ & $\begin{array}{l}1.2 \\
7 \\
\end{array}$ & $\begin{array}{l}1.2 \\
9 \\
\end{array}$ & 1.10 & $\begin{array}{l}0.5 \\
1 \\
\end{array}$ & $\begin{array}{l}0.8 \\
0 \\
\end{array}$ & $\begin{array}{l}0.9 \\
3 \\
\end{array}$ & $\begin{array}{l}1.0 \\
1 \\
\end{array}$ & $\begin{array}{l}1.0 \\
5 \\
\end{array}$ & $\begin{array}{l}1.1 \\
1 \\
\end{array}$ & $\begin{array}{l}1.1 \\
5 \\
\end{array}$ & $\begin{array}{l}1.1 \\
8 \\
\end{array}$ & $\begin{array}{l}1.2 \\
1 \\
\end{array}$ & $\begin{array}{l}1.2 \\
3 \\
\end{array}$ & $\begin{array}{l}1.2 \\
4 \\
\end{array}$ \\
\hline $\begin{array}{l}\text { Diethyleth } \\
\text { er }\end{array}$ & 8 & 6.70 & $\begin{array}{l}2.9 \\
5 \\
\end{array}$ & $\begin{array}{l}1.5 \\
4 \\
\end{array}$ & $\begin{array}{l}1.2 \\
6 \\
\end{array}$ & $\begin{array}{l}1.2 \\
5 \\
\end{array}$ & $\begin{array}{l}1.2 \\
8 \\
\end{array}$ & $\begin{array}{l}1.3 \\
3 \\
\end{array}$ & $\begin{array}{l}1.4 \\
0 \\
\end{array}$ & $\begin{array}{l}1.4 \\
5 \\
\end{array}$ & $\begin{array}{l}1.5 \\
1 \\
\end{array}$ & $\begin{array}{l}1.5 \\
6 \\
\end{array}$ & $\begin{array}{l}1.6 \\
0 \\
\end{array}$ & 6.94 & $\begin{array}{l}3.0 \\
2 \\
\end{array}$ & $\begin{array}{l}1.5 \\
8 \\
\end{array}$ & $\begin{array}{l}1.2 \\
9 \\
\end{array}$ & $\begin{array}{l}1.2 \\
2 \\
\end{array}$ & $\begin{array}{l}1.2 \\
6 \\
\end{array}$ & $\begin{array}{l}1.2 \\
9 \\
\end{array}$ & $\begin{array}{l}1.3 \\
7 \\
\end{array}$ & $\begin{array}{l}1.4 \\
2 \\
\end{array}$ & $\begin{array}{l}1.4 \\
8 \\
\end{array}$ & $\begin{array}{l}1.5 \\
3 \\
\end{array}$ & $\begin{array}{l}1.5 \\
6 \\
\end{array}$ \\
\hline Heptane & 6 & 8.54 & $\begin{array}{l}3.0 \\
9 \\
\end{array}$ & $\begin{array}{l}1.5 \\
4 \\
\end{array}$ & $\begin{array}{l}2.0 \\
8 \\
\end{array}$ & $\begin{array}{l}2.9 \\
7 \\
\end{array}$ & $\begin{array}{l}3.5 \\
8 \\
\end{array}$ & $\begin{array}{l}3.9 \\
9 \\
\end{array}$ & $\begin{array}{l}4.3 \\
0 \\
\end{array}$ & $\begin{array}{l}4.5 \\
3 \\
\end{array}$ & $\begin{array}{l}4.7 \\
0 \\
\end{array}$ & $\begin{array}{l}4.8 \\
4 \\
\end{array}$ & $\begin{array}{l}4.9 \\
5 \\
\end{array}$ & 8.77 & $\begin{array}{l}3.2 \\
2 \\
\end{array}$ & $\begin{array}{l}1.7 \\
1 \\
\end{array}$ & $\begin{array}{l}2.1 \\
7 \\
\end{array}$ & $\begin{array}{l}3.0 \\
1 \\
\end{array}$ & $\begin{array}{l}3.6 \\
0 \\
\end{array}$ & $\begin{array}{l}4.0 \\
1 \\
\end{array}$ & $\begin{array}{l}4.3 \\
1 \\
\end{array}$ & $\begin{array}{l}4.5 \\
3 \\
\end{array}$ & $\begin{array}{l}4.7 \\
0 \\
\end{array}$ & $\begin{array}{l}4.8 \\
3 \\
\end{array}$ & $\begin{array}{l}4.9 \\
4 \\
\end{array}$ \\
\hline Octanol & $\begin{array}{l}10 \\
3 \\
\end{array}$ & 7.49 & $\begin{array}{l}6.3 \\
0\end{array}$ & $\begin{array}{l}5.7 \\
8 \\
\end{array}$ & $\begin{array}{l}5.5 \\
1 \\
\end{array}$ & $\begin{array}{l}5.3 \\
4\end{array}$ & $\begin{array}{l}5.2 \\
3\end{array}$ & $\begin{array}{l}5.1 \\
6 \\
\end{array}$ & $\begin{array}{l}5.1 \\
1 \\
\end{array}$ & $\begin{array}{l}5.0 \\
7 \\
\end{array}$ & $\begin{array}{l}5.0 \\
4 \\
\end{array}$ & $\begin{array}{l}5.0 \\
2 \\
\end{array}$ & $\begin{array}{l}5.0 \\
0\end{array}$ & 7.52 & $\begin{array}{l}6.2 \\
9 \\
\end{array}$ & $\begin{array}{l}5.7 \\
6 \\
\end{array}$ & $\begin{array}{l}5.4 \\
8 \\
\end{array}$ & $\begin{array}{l}5.3 \\
2 \\
\end{array}$ & $\begin{array}{l}5.2 \\
2 \\
\end{array}$ & $\begin{array}{l}5.1 \\
5 \\
\end{array}$ & $\begin{array}{l}5.1 \\
0\end{array}$ & $\begin{array}{l}5.0 \\
6 \\
\end{array}$ & $\begin{array}{l}5.0 \\
3 \\
\end{array}$ & $\begin{array}{l}5.0 \\
1 \\
\end{array}$ & $\begin{array}{l}4.9 \\
9 \\
\end{array}$ \\
\hline All & $\begin{array}{l}13 \\
9 \\
\end{array}$ & 7.32 & $\begin{array}{l}5.4 \\
1 \\
\end{array}$ & $\begin{array}{l}4.6 \\
9 \\
\end{array}$ & $\begin{array}{l}4.4 \\
6 \\
\end{array}$ & $\begin{array}{l}4.3 \\
6 \\
\end{array}$ & $\begin{array}{l}4.3 \\
2 \\
\end{array}$ & $\begin{array}{l}4.3 \\
1 \\
\end{array}$ & $\begin{array}{l}4.3 \\
1 \\
\end{array}$ & $\begin{array}{l}4.3 \\
0\end{array}$ & $\begin{array}{l}4.3 \\
0 \\
\end{array}$ & $\begin{array}{l}4.3 \\
0\end{array}$ & $\begin{array}{l}4.3 \\
0\end{array}$ & 7.36 & $\begin{array}{l}5.4 \\
0\end{array}$ & $\begin{array}{l}4.6 \\
9\end{array}$ & $\begin{array}{l}4.4 \\
4 \\
\end{array}$ & $\begin{array}{l}4.3 \\
5 \\
\end{array}$ & $\begin{array}{l}4.3 \\
2 \\
\end{array}$ & $\begin{array}{l}4.3 \\
0 \\
\end{array}$ & $\begin{array}{l}4.2 \\
9 \\
\end{array}$ & $\begin{array}{l}4.2 \\
9 \\
\end{array}$ & $\begin{array}{l}4.2 \\
9 \\
\end{array}$ & $\begin{array}{l}4.2 \\
9 \\
\end{array}$ & $\begin{array}{l}4.2 \\
9 \\
\end{array}$ \\
\hline
\end{tabular}

Table S5. Mean unsigned errors (unit in $\mathrm{kcal} / \mathrm{mol}$ ) of 283 solvation free energies of ions calculated using IEFPCM solvation model.

\begin{tabular}{|c|c|c|c|c|c|c|c|c|c|c|c|c|c|c|c|c|c|c|c|}
\hline \multirow[b]{2}{*}{$\begin{array}{c}\text { Solute } \\
\text { class }\end{array}$} & \multirow[b]{2}{*}{$\mathrm{N}$} & \multicolumn{9}{|c|}{ UAHF } & \multicolumn{9}{|c|}{ UAKS } \\
\hline & & $\begin{array}{l}\text { Alpha=1. } \\
0\end{array}$ & 1.1 & 1.2 & 1.3 & 1.4 & 1.5 & 1.6 & 1.7 & 1.8 & $\begin{array}{l}\text { Alpha=1. } \\
0\end{array}$ & 1.1 & 1.2 & 1.3 & 1.4 & 1.5 & 1.6 & 1.7 & 1.8 \\
\hline \multicolumn{20}{|c|}{ Acetonitrile } \\
\hline
\end{tabular}




\begin{tabular}{|c|c|c|c|c|c|c|c|c|c|c|c|c|c|c|c|c|c|c|c|}
\hline $\begin{array}{c}\text { CHNO } \\
\text { Cation } \\
\text { s }\end{array}$ & 36 & 25.15 & 5.97 & $\begin{array}{l}10.2 \\
2\end{array}$ & $\begin{array}{l}17.4 \\
9\end{array}$ & $\begin{array}{l}22.8 \\
4\end{array}$ & $\begin{array}{l}26.9 \\
9\end{array}$ & \multirow{3}{*}{\multicolumn{3}{|c|}{ N/A }} & 23.59 & 6.38 & $\begin{array}{l}10.4 \\
7\end{array}$ & $\begin{array}{l}17.7 \\
7\end{array}$ & $\begin{array}{l}23.0 \\
9\end{array}$ & $\begin{array}{l}27.1 \\
9\end{array}$ & & \multirow{7}{*}{ N/A } & \\
\hline $\begin{array}{c}\text { Other } \\
\text { Cation } \\
\text { s }\end{array}$ & 3 & 27.24 & $\begin{array}{l}10.1 \\
4\end{array}$ & $\begin{array}{l}17.6 \\
6\end{array}$ & $\begin{array}{l}24.7 \\
9\end{array}$ & $\begin{array}{l}29.8 \\
8\end{array}$ & $\begin{array}{l}33.8 \\
7\end{array}$ & & & & 27.54 & $\begin{array}{l}10.7 \\
2\end{array}$ & $\begin{array}{l}17.7 \\
9\end{array}$ & $\begin{array}{l}24.8 \\
7\end{array}$ & $\begin{array}{l}29.9 \\
3\end{array}$ & $\begin{array}{l}33.9 \\
0\end{array}$ & & & \\
\hline $\begin{array}{c}\text { All } \\
\text { Cation } \\
\text { s }\end{array}$ & 39 & 25.31 & 6.29 & $\begin{array}{l}10.7 \\
9\end{array}$ & $\begin{array}{l}18.0 \\
5\end{array}$ & $\begin{array}{l}23.3 \\
8\end{array}$ & $\begin{array}{l}27.5 \\
2\end{array}$ & & & & 25.56 & 6.72 & $\begin{array}{l}11.0 \\
3\end{array}$ & $\begin{array}{l}18.3 \\
1\end{array}$ & $\begin{array}{l}23.6 \\
1\end{array}$ & $\begin{array}{l}27.7 \\
1\end{array}$ & & & \\
\hline $\begin{array}{l}\text { CHNO } \\
\text { Anions }\end{array}$ & 19 & 24.02 & $\begin{array}{l}16.4 \\
9 \\
\end{array}$ & $\begin{array}{l}10.8 \\
6 \\
\end{array}$ & 5.97 & 2.46 & 2.42 & 5.28 & 8.12 & $\begin{array}{l}10.6 \\
4 \\
\end{array}$ & 19.54 & $\begin{array}{l}13.0 \\
2 \\
\end{array}$ & 8.15 & 4.09 & 2.40 & 3.99 & & & \\
\hline $\begin{array}{l}\text { Other } \\
\text { Anions }\end{array}$ & 11 & 37.13 & \begin{tabular}{|l|}
26.4 \\
8 \\
\end{tabular} & $\begin{array}{l}14.5 \\
4 \\
\end{array}$ & 6.97 & 2.47 & 2.73 & \multirow{3}{*}{\multicolumn{3}{|c|}{ N/A }} & 34.11 & $\begin{array}{l}24.1 \\
0 \\
\end{array}$ & $\begin{array}{l}12.6 \\
1 \\
\end{array}$ & 5.43 & 2.59 & 3.72 & & & \\
\hline $\begin{array}{c}\text { All } \\
\text { Anions }\end{array}$ & 30 & 28.83 & \begin{tabular}{|l|}
20.1 \\
5 \\
\end{tabular} & $\begin{array}{l}12.2 \\
1\end{array}$ & 6.33 & 2.46 & 2.53 & & & & 24.88 & $\begin{array}{l}17.0 \\
8\end{array}$ & 9.79 & 4.58 & 2.47 & 3.89 & & & \\
\hline $\begin{array}{c}\text { All } \\
\text { lons }\end{array}$ & 69 & 26.84 & \begin{tabular}{|l|}
12.3 \\
1 \\
\end{tabular} & $\begin{array}{l}11.4 \\
1 \\
\end{array}$ & $\begin{array}{l}12.9 \\
6 \\
\end{array}$ & $\begin{array}{l}14.2 \\
9 \\
\end{array}$ & $\begin{array}{l}16.6 \\
6 \\
\end{array}$ & & & & 25.27 & $\begin{array}{l}11.2 \\
2 \\
\end{array}$ & $\begin{array}{l}10.4 \\
9 \\
\end{array}$ & $\begin{array}{l}12.3 \\
4 \\
\end{array}$ & $\begin{array}{l}14.4 \\
2 \\
\end{array}$ & $\begin{array}{l}17.3 \\
5 \\
\end{array}$ & & & \\
\hline \multicolumn{20}{|c|}{ Dimethylsulfoxide } \\
\hline $\begin{array}{c}\text { CHNO } \\
\text { Cation } \\
\mathrm{s} \\
\end{array}$ & 4 & 12.06 & 3.35 & $\begin{array}{l}12.3 \\
9\end{array}$ & $\begin{array}{l}18.6 \\
6\end{array}$ & $\begin{array}{l}23.4 \\
8\end{array}$ & $\begin{array}{l}27.4 \\
1\end{array}$ & $\begin{array}{l}30.7 \\
3\end{array}$ & $\begin{array}{l}33.6 \\
1\end{array}$ & $\begin{array}{l}36.1 \\
2\end{array}$ & 13.16 & 2.87 & $\begin{array}{l}12.1 \\
2\end{array}$ & $\begin{array}{l}18.4 \\
9\end{array}$ & $\begin{array}{l}23.3 \\
6\end{array}$ & $\begin{array}{l}27.3 \\
2\end{array}$ & $\begin{array}{l}30.6 \\
6\end{array}$ & $\begin{array}{l}33.5 \\
5\end{array}$ & $\begin{array}{l}36.0 \\
8\end{array}$ \\
\hline $\begin{array}{c}\text { Other } \\
\text { Cation } \\
\text { s }\end{array}$ & 0 & \multicolumn{18}{|c|}{ N/A } \\
\hline $\begin{array}{c}\text { All } \\
\text { Cation } \\
\text { s }\end{array}$ & 4 & 12.06 & 3.35 & $\begin{array}{l}12.3 \\
9\end{array}$ & $\begin{array}{l}18.6 \\
6\end{array}$ & $\begin{array}{l}23.4 \\
8\end{array}$ & $\begin{array}{l}27.4 \\
1\end{array}$ & $\begin{array}{l}30.7 \\
3\end{array}$ & $\begin{array}{l}33.6 \\
1\end{array}$ & $\begin{array}{l}36.1 \\
2\end{array}$ & 13.16 & 2.87 & $\begin{array}{l}12.1 \\
2\end{array}$ & $\begin{array}{l}18.4 \\
9\end{array}$ & $\begin{array}{l}23.3 \\
6\end{array}$ & $\begin{array}{l}27.3 \\
2\end{array}$ & $\begin{array}{l}30.6 \\
6\end{array}$ & $\begin{array}{l}33.5 \\
5\end{array}$ & $\begin{array}{l}36.0 \\
8\end{array}$ \\
\hline $\begin{array}{l}\mathrm{CHNO} \\
\text { Anions }\end{array}$ & 52 & 244.73 & \begin{tabular}{|l|}
74.2 \\
8 \\
\end{tabular} & $\begin{array}{l}21.5 \\
4 \\
\end{array}$ & $\begin{array}{l}10.5 \\
4 \\
\end{array}$ & 6.01 & 4.05 & 5.00 & 7.71 & $\begin{array}{l}10.3 \\
1 \\
\end{array}$ & 242.83 & $\begin{array}{l}72.6 \\
2 \\
\end{array}$ & $\begin{array}{l}20.3 \\
9 \\
\end{array}$ & 9.78 & 5.43 & 4.25 & 5.50 & 7.89 & $\begin{array}{l}10.3 \\
8 \\
\end{array}$ \\
\hline $\begin{array}{l}\text { Other } \\
\text { Anions }\end{array}$ & 15 & 89.51 & $\begin{array}{l}55.2 \\
9\end{array}$ & $\begin{array}{l}29.7 \\
9 \\
\end{array}$ & $\begin{array}{l}13.4 \\
1 \\
\end{array}$ & 7.06 & 3.59 & 4.23 & 6.88 & 9.37 & 87.67 & $\begin{array}{l}53.8 \\
2 \\
\end{array}$ & $\begin{array}{l}28.5 \\
2 \\
\end{array}$ & $\begin{array}{l}12.3 \\
0 \\
\end{array}$ & 6.10 & 3.75 & 4.49 & 7.14 & 9.59 \\
\hline $\begin{array}{c}\text { All } \\
\text { Anions }\end{array}$ & 67 & 209.98 & \begin{tabular}{|l|}
70.0 \\
3 \\
\end{tabular} & $\begin{array}{l}23.3 \\
9 \\
\end{array}$ & $\begin{array}{l}11.1 \\
8 \\
\end{array}$ & 6.25 & 3.94 & 4.83 & 7.53 & $\begin{array}{l}10.1 \\
0 \\
\end{array}$ & 208.09 & $\begin{array}{l}68.4 \\
1 \\
\end{array}$ & $\begin{array}{l}22.2 \\
1 \\
\end{array}$ & $\begin{array}{l}10.3 \\
5 \\
\end{array}$ & 5.58 & 4.14 & 5.27 & 7.72 & $\begin{array}{l}10.2 \\
0 \\
\end{array}$ \\
\hline
\end{tabular}




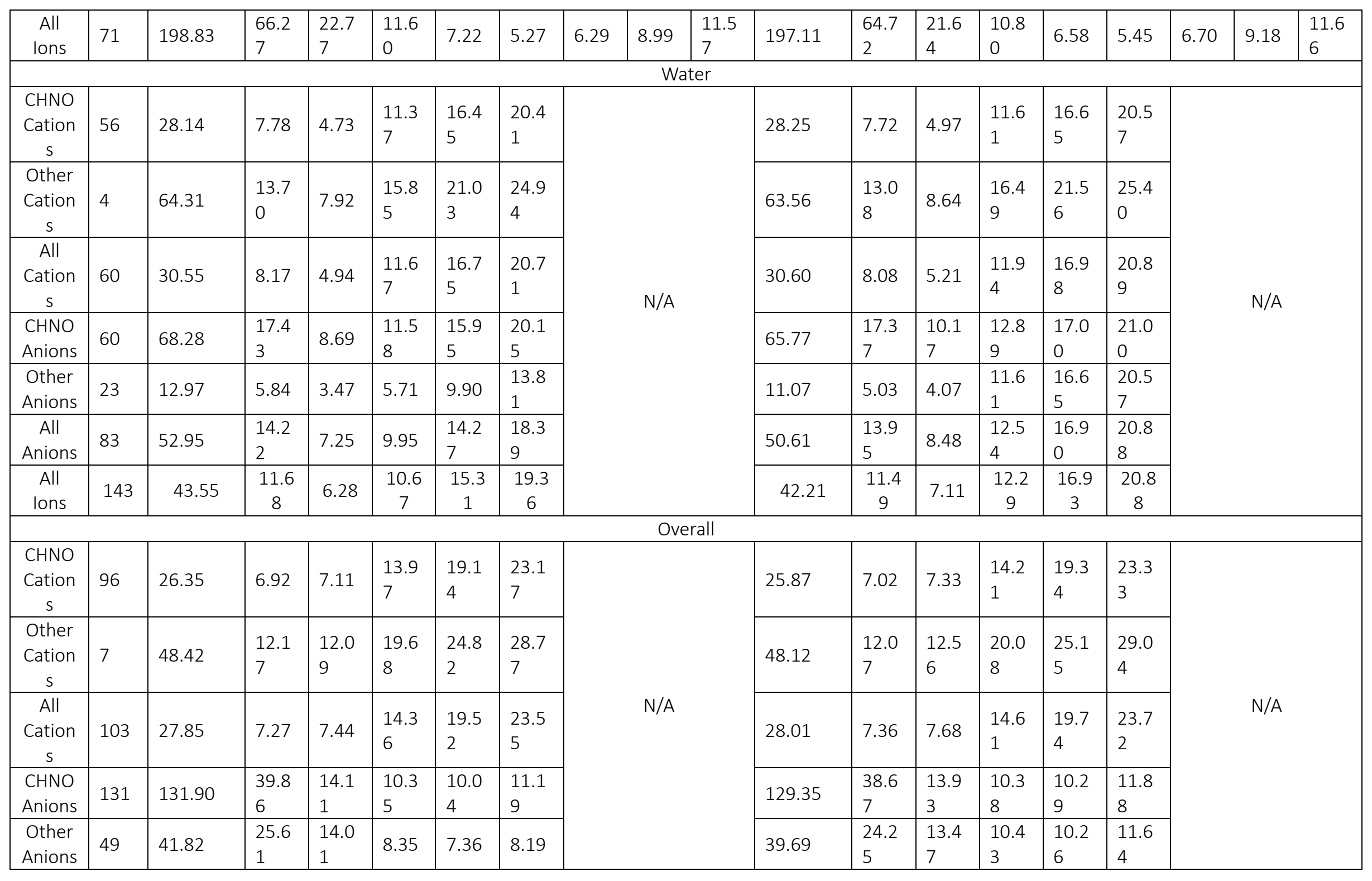




\begin{tabular}{|c|c|c|c|c|c|c|c|c|c|c|c|c|c|c|}
\hline $\begin{array}{c}\text { All } \\
\text { Anions }\end{array}$ & 180 & 107.38 & $\begin{array}{l}35.9 \\
8 \\
\end{array}$ & $\begin{array}{l}14.1 \\
1 \\
\end{array}$ & 9.80 & 9.32 & $\begin{array}{l}10.3 \\
7 \\
\end{array}$ & 104.94 & $\begin{array}{l}34.7 \\
4\end{array}$ & $\begin{array}{l}13.8 \\
1 \\
\end{array}$ & $\begin{array}{l}10.4 \\
0\end{array}$ & $\begin{array}{l}10.2 \\
8\end{array}$ & $\begin{array}{l}11.8 \\
2 \\
\end{array}$ & \\
\hline $\begin{array}{c}\text { All } \\
\text { Ions }\end{array}$ & 283 & 78.43 & $\begin{array}{c}25.5 \\
3\end{array}$ & $\begin{array}{c}11.6 \\
7\end{array}$ & $\begin{array}{c}11.4 \\
6\end{array}$ & $\begin{array}{c}13.0 \\
3\end{array}$ & $\begin{array}{c}15.1 \\
7\end{array}$ & 76.94 & $\begin{array}{c}24.7 \\
8\end{array}$ & $\begin{array}{c}11.6 \\
0\end{array}$ & $\begin{array}{c}11.9 \\
3\end{array}$ & \begin{tabular}{|c}
13.7 \\
2
\end{tabular} & $\begin{array}{c}16.1 \\
5\end{array}$ & \\
\hline $\begin{array}{c}\text { All } \\
\text { IEFPC } \\
\text { M } \\
\text { Data }\end{array}$ & $\begin{array}{l}171 \\
9\end{array}$ & 19.71 & 6.97 & 4.39 & 5.10 & $\mathrm{~N} / \mathrm{A}$ & $\mathrm{N} / \mathrm{A}$ & 19.19 & 6.75 & 4.42 & 5.28 & N/A & $\mathrm{N} / \mathrm{A}$ & \\
\hline
\end{tabular}

Table S6. Mean unsigned errors (unit in $\mathrm{kcal} / \mathrm{mol}$ ) of 283 solvation free energies of ions calculated using CPCM solvation model.

\begin{tabular}{|c|c|c|c|c|c|c|c|c|c|c|c|c|c|c|c|c|c|c|c|}
\hline \multirow{2}{*}{$\begin{array}{l}\text { Solute } \\
\text { class }\end{array}$} & \multirow[b]{2}{*}{ N } & \multicolumn{9}{|c|}{ UAHF } & \multicolumn{9}{|c|}{ UAKS } \\
\hline & & $\begin{array}{l}\text { Alpha=1. } \\
0\end{array}$ & 1.1 & 1.2 & 1.3 & 1.4 & 1.5 & 1.6 & 1.7 & 1.8 & $\begin{array}{l}\text { Alpha=1. } \\
0\end{array}$ & 1.1 & 1.2 & 1.3 & 1.4 & 1.5 & 1.6 & 1.7 & 1.8 \\
\hline \multicolumn{20}{|c|}{ Acetonitrile } \\
\hline $\begin{array}{c}\text { CHNO } \\
\text { Cation } \\
\mathrm{s}\end{array}$ & 36 & 25.94 & 6.04 & 9.96 & $\begin{array}{l}17.2 \\
9\end{array}$ & $\begin{array}{l}22.6 \\
9\end{array}$ & $\begin{array}{l}26.8 \\
7\end{array}$ & \multirow{3}{*}{\multicolumn{3}{|c|}{ N/A }} & 26.19 & 6.43 & $\begin{array}{l}10.2 \\
1\end{array}$ & $\begin{array}{l}17.5 \\
7\end{array}$ & $\begin{array}{l}22.9 \\
3\end{array}$ & $\begin{array}{l}27.0 \\
7\end{array}$ & & \multirow{7}{*}{$\mathrm{N} / \mathrm{A}$} & \\
\hline $\begin{array}{c}\text { Other } \\
\text { Cation } \\
\mathrm{s}\end{array}$ & 3 & 27.78 & $\begin{array}{l}10.1 \\
5\end{array}$ & $\begin{array}{l}17.5 \\
0\end{array}$ & $\begin{array}{l}24.6 \\
9\end{array}$ & $\begin{array}{l}29.8 \\
1\end{array}$ & $\begin{array}{l}33.8 \\
2\end{array}$ & & & & 28.09 & $\begin{array}{l}10.7 \\
4\end{array}$ & $\begin{array}{l}17.6 \\
3\end{array}$ & $\begin{array}{l}24.7 \\
7\end{array}$ & $\begin{array}{l}29.8 \\
6\end{array}$ & $\begin{array}{l}33.8 \\
5\end{array}$ & & & \\
\hline $\begin{array}{c}\text { All } \\
\text { Cation } \\
\text { s }\end{array}$ & 39 & 26.08 & 6.35 & $\begin{array}{l}10.5 \\
4\end{array}$ & $\begin{array}{l}17.8 \\
6\end{array}$ & $\begin{array}{l}23.2 \\
3\end{array}$ & $\begin{array}{l}27.4 \\
0\end{array}$ & & & & 26.34 & 6.76 & $\begin{array}{l}10.7 \\
8\end{array}$ & $\begin{array}{l}18.1 \\
2\end{array}$ & $\begin{array}{l}23.4 \\
7\end{array}$ & $\begin{array}{l}27.5 \\
9\end{array}$ & & & \\
\hline $\begin{array}{l}\text { CHNO } \\
\text { Anions }\end{array}$ & 19 & 24.45 & $\begin{array}{l}16.7 \\
7\end{array}$ & $\begin{array}{l}11.0 \\
7\end{array}$ & 6.14 & 2.54 & 2.35 & 5.16 & 8.01 & $\begin{array}{l}10.5 \\
4\end{array}$ & 19.92 & $\begin{array}{l}13.2 \\
6\end{array}$ & 8.33 & 4.21 & 2.36 & 3.90 & & & \\
\hline $\begin{array}{l}\text { Other } \\
\text { Anions }\end{array}$ & 11 & 38.00 & $\begin{array}{l}27.0 \\
8\end{array}$ & $\begin{array}{l}14.8 \\
4\end{array}$ & 7.14 & 2.58 & 2.63 & \multirow{3}{*}{\multicolumn{3}{|c|}{ N/A }} & 34.94 & $\begin{array}{l}24.6 \\
7 \\
\end{array}$ & $\begin{array}{l}12.8 \\
9\end{array}$ & 5.56 & 2.54 & 3.64 & & & \\
\hline $\begin{array}{c}\text { All } \\
\text { Anions }\end{array}$ & 30 & 29.42 & $\begin{array}{l}20.5 \\
5 \\
\end{array}$ & $\begin{array}{l}12.4 \\
5 \\
\end{array}$ & 6.51 & 2.55 & 2.45 & & & & 25.43 & $\begin{array}{l}17.4 \\
4 \\
\end{array}$ & $\begin{array}{l}10.0 \\
0\end{array}$ & 4.71 & 2.43 & 3.80 & & & \\
\hline $\begin{array}{l}\text { All } \\
\text { Ions }\end{array}$ & 69 & 27.53 & $\begin{array}{l}12.5 \\
2\end{array}$ & $\begin{array}{l}11.3 \\
7\end{array}$ & $\begin{array}{l}12.9 \\
2\end{array}$ & $\begin{array}{l}14.2 \\
4\end{array}$ & $\begin{array}{l}16.5 \\
5\end{array}$ & & & & 25.94 & $\begin{array}{l}11.4 \\
1\end{array}$ & $\begin{array}{l}10.4 \\
4\end{array}$ & $\begin{array}{l}12.2 \\
9\end{array}$ & $\begin{array}{l}14.3 \\
2\end{array}$ & $\begin{array}{l}17.2 \\
5\end{array}$ & & & \\
\hline
\end{tabular}




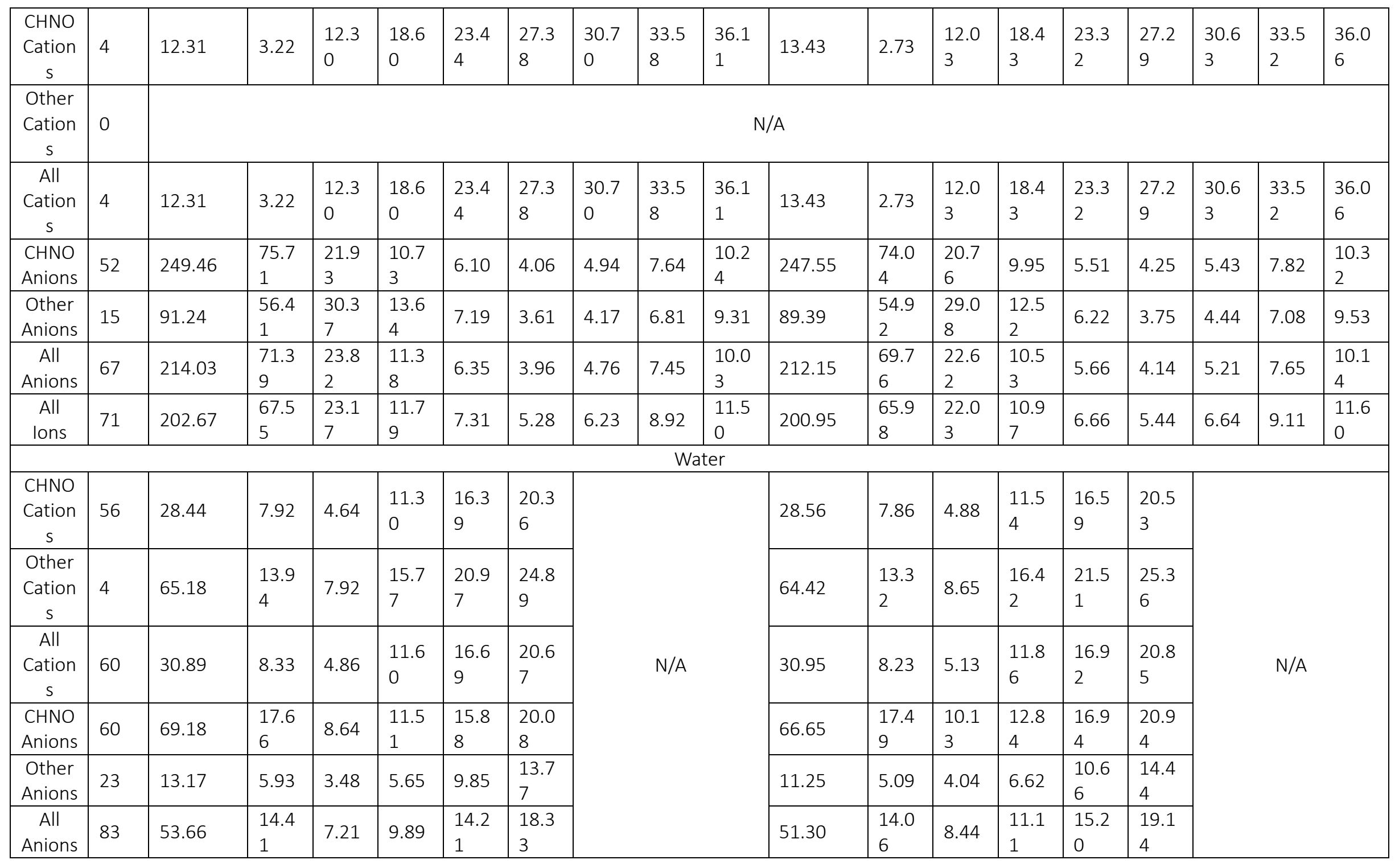




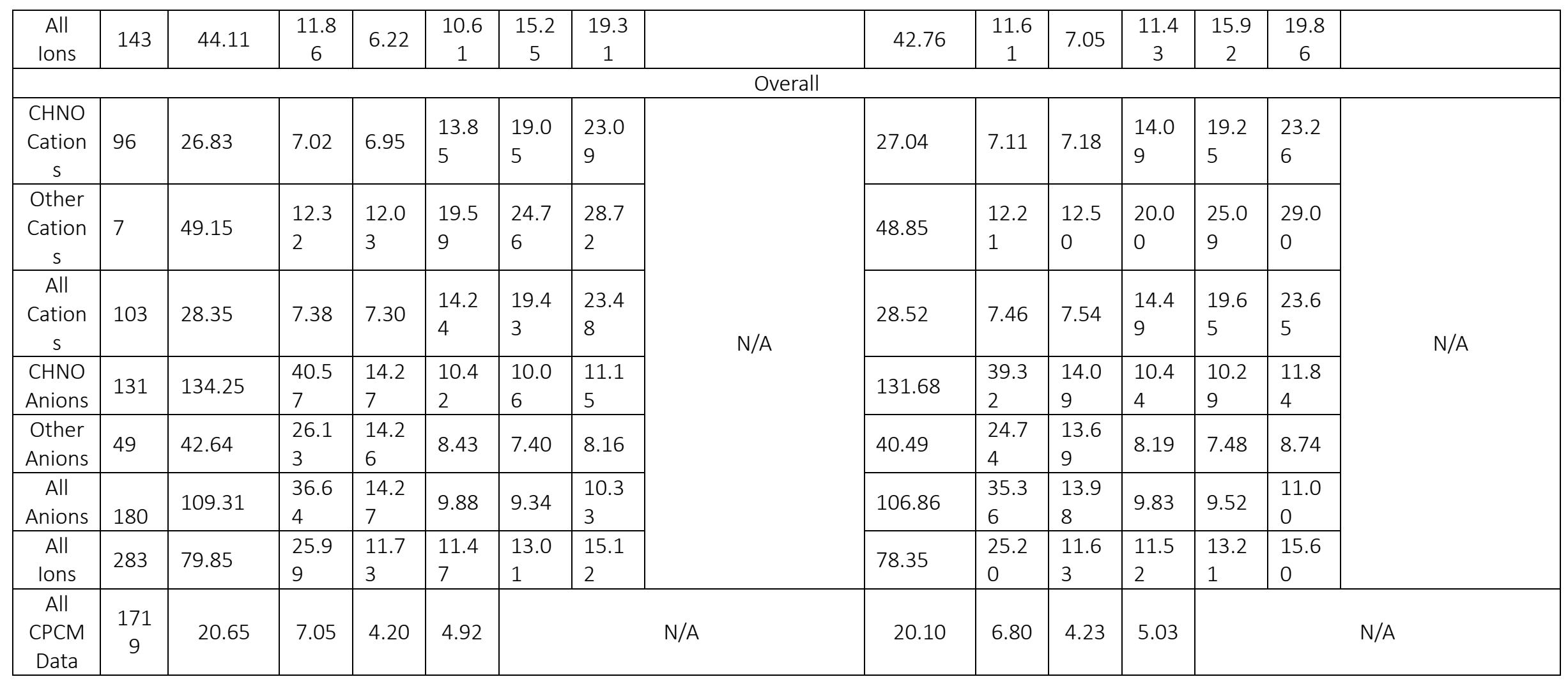




\section{Section S2. Raw data in pKa calculations}

Note: More details can be seen in the spreadsheet of supporting information materials. Here we only present data used in Figure $2 \sim$ Figure 5 in main text.

Table S7. Data used in Figure 2

\begin{tabular}{|l|l|l|l|}
\hline & IEFPCM-UAHF-1.2 & IEFPCM-UAKS-1.2 & CPCM-UAHF-1.2 \\
\hline A1-A5 & 0.61 & 0.61 & 0.61 \\
\hline T1-T5 & 1.52 & 0.38 & 1.56 \\
\hline CA1-CA5 & 4.73 & 4.21 & 4.73 \\
\hline PY1-PY5 & 2.53 & 1.79 & 2.52 \\
\hline AL1-AL5 & 5.9 & 8.7 & 5.82 \\
\hline AN1-AN5 & 2.87 & 4.2 & 2.84 \\
\hline C1-C5 & 1.02 & 3.21 & 0.38 \\
\hline N1-N5 & 7.67 & 7.34 & 1.02 \\
\hline P1-P5 & 3.09 & 5.46 & 7.67 \\
\hline Overall & 3.33 & 3.99 & 3.06 \\
\hline
\end{tabular}

Table S8. Data used in Figure 3

\begin{tabular}{|l|l|l|l|}
\hline & IEFPCM-UAHF-MIXED & IEFPCM-UAKS-MIXED & CPCM-UAHF-MIXED \\
\hline PH1-PH5 & 4.38 & 5.47 & 4.32 \\
\hline CAR1-CAR5 & 2.56 & 2.88 & 2.53 \\
\hline CARB1-CARB5 & 5.77 & 6.44 & 5.74 \\
\hline PYR1-PYR5 & 5.04 & 3.2 & 4.96 \\
\hline Overall & 4.44 & 4.50 & 2.85 \\
\hline
\end{tabular}

Table S9. Data used in Figure 4

\begin{tabular}{|l|l|l|l|l|}
\hline & IEFPCM-UAHF & IEFPCM-UAKS & CPCM-UAHF \\
\hline$E S F=1.1$ & 4.09 & 4.67 & 4.09 & \\
\hline$E S F=1.2$ & 3.33 & 3.99 & 3.31 & \\
\hline$E S F=1.3$ & 5.32 & 5.52 & 5.67 & \\
\hline
\end{tabular}


Table S10. Data used in Figure 5

\begin{tabular}{|l|l|l|l|l|l|l|l|}
\hline $1-0-3$ & $1-1-4$ & $1-2-5$ & $2-0-3$ & $2-1-4$ & $2-2-5$ & $3-0-3$ & $3-1-4$ \\
\hline 4.82 & 3.06 & 4.40 & 7.88 & 4.52 & 4.73 & 9.91 & 5.90 \\
\hline
\end{tabular}

Table S11. Overall $\mathrm{p} K_{\mathrm{a}}$ MAEs in acetonitrile obtained with different ESF combinations using IEFPCM-UAHF, CPCM-UAHF and CPCM-UAKS methods.

\begin{tabular}{|c|c|c|c|c|c|c|c|c|}
\hline \multicolumn{9}{|c|}{ IEFPCM-UAHF } \\
\hline $1-0-3$ & 1-1-4 & $1-2-5$ & $2-0-3$ & 2-1-4 & $2-2-5$ & $3-0-3$ & $3-1-4$ & $3-2-5$ \\
\hline 4.35 & 3.74 & 6.90 & 5.93 & 2.47 & 3.00 & 7.52 & 3.33 & 3.19 \\
\hline \multicolumn{9}{|c|}{ CPCM-UAHF } \\
\hline $1-0-4$ & $1-1-5$ & $1-2-6$ & $2-0-4$ & $2-1-5$ & $2-2-6$ & $3-0-4$ & $3-1-5$ & $3-2-6$ \\
\hline 4.94 & 5.66 & 8.66 & 4.66 & 1.72 & 4.40 & 5.93 & 2.09 & 2.42 \\
\hline \multicolumn{9}{|c|}{ CPCM-UAKS } \\
\hline $1-0-3$ & 1-1-4 & $1-2-5$ & $2-0-3$ & $2-1-4$ & $2-2-5$ & $3-0-3$ & $3-1-4$ & $3-2-5$ \\
\hline 4.53 & 3.22 & 6.51 & 6.33 & 1.74 & 2.56 & 7.92 & 3.17 & 2.38 \\
\hline
\end{tabular}

Table S12. Overall $\mathrm{p} K_{\mathrm{a}} \mathrm{MAEs}$ in acetonitrile obtained with $\mathrm{ESF}=1.1$.

\begin{tabular}{|l|l|l|l|}
\hline & IEFPCM-UAHF & IEFPCM-UAKS & CPCM-UAHF \\
\hline PH1-PH5 & 7.19 & 5.35 & 7.16 \\
\hline CAR1-CAR5 & 2.60 & 2.87 & 2.61 \\
\hline CARB1-CARB5 & 10.41 & 8.38 & 5.30 \\
\hline PYR1-PYR5 & 3.17 & 1.27 & 2.84 \\
\hline
\end{tabular}


Section S3. Optimized structures of 65 molecules

A1-BASE.xyz-M062X-MG3S

$1 \backslash 1 \backslash G I N C-R 560 \backslash F O p t \backslash R M 062 X \backslash G e n \backslash C 4 H 11 N 1 \backslash R O O T \backslash 17-J u n-2019 \backslash 0 \backslash \backslash \#$ M062X/Gen SCF=Tight INT(grid=ultrafine) OPT IOP(2/17=4)\\title \\0,1\N,-0.0000000 $481,-0.272016515,-0.08481575 \backslash C,-1.2150946341,0.5208718274,0.0055795778$ $\backslash C, 1.2150945407,0.5208718232,0.0055795778 \backslash C,-2.4450801311,-0.368889909$ $1,-0.0222868439 \backslash \mathrm{C}, 2.4450800345,-0.3688899177,-0.0222868439 \backslash \mathrm{H},-1.229828$ $4331,1.1516192397,0.9078920246 \backslash \mathrm{H},-1.2299335978,1.2016826926,-0.8491187$ $635 \backslash H, 1.2299335067,1.2016826883,-0.8491187635 \backslash H, 1.2298283419,1.1516192$ $354,0.9078920246 \backslash \mathrm{H},-0.0000000493,-0.9791424424,0.6425143211 \backslash \mathrm{H},-2.45997$ $12208,-0.9656251018,-0.9330579843 \backslash \mathrm{H},-2.4474456761,-1.0511249004,0.8296$ $485195 \backslash \mathrm{H},-3.3575094889,0.2239971301,0.0237006843 \backslash \mathrm{H}, 2.4474455772,-1.051$ $124909,0.8296485195 \backslash \mathrm{H}, 2.4599711222,-0.9656251104,-0.9330579843 \backslash \mathrm{H}, 3.357$ 5093944,0.2239971184,0.0237006843\\Version=ES64L-G16RevB.01\State=1-A' $\backslash \mathrm{HF}=-213.7547253 \backslash \mathrm{RMSD}=6.206 \mathrm{e}-09 \backslash \mathrm{RMSF}=1.234 \mathrm{e}-05 \backslash \mathrm{Dipole}=0 ., 0.1469284,0.3$ 387427\Quadrupole=0.6071347,-0.3398079,-0.2673268,0.,0.,-1.2838978\PG= CS [SG(H1N1),X(C4H10)]\\@

A1.xyz-M062X-MG3S

$1 \backslash 1 \backslash G I N C-R 653 \backslash F O p t \backslash R M 062 X \backslash G e n \backslash C 4 H 12 N 1(1+) \backslash R O O T \backslash 17-J u n-2019 \backslash 0 \backslash \backslash \#$ M062X/

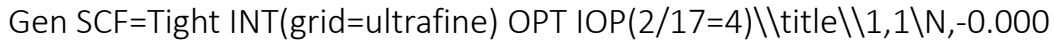
0000897,-0.2623555538,0.0000405168\C,1.2684423054,0.5485004622,0.00002 $9278 \backslash C,-1.268442046,0.5485005294,0.0000273846 \backslash C, 2.4822715122,-0.354730$ $8465,0.0000326642 \backslash \mathrm{C},-2.4822714305,-0.3547305817,-0.0000743414 \backslash \mathrm{H}, 1.2229$ $885617,1.1816258539,-0.8835889721 \backslash H, 1.2229943646,1.1816530663,0.883630$ $0021 \backslash \mathrm{H},-1.223030909,1.1815843103,0.8836774588 \backslash \mathrm{H},-1.2229516332,1.181695$ $0603,-0.8835413676 \backslash \mathrm{H},-0.0000100878,-0.8811294147,-0.814535724 \backslash \mathrm{H}, 2.5112$ $06585,-0.9858993269,0.8877922025 \backslash H, 2.5110213635,-0.9861652295,-0.88754$ $43399 \backslash \mathrm{H}, 3.3815886709,0.2570462764,-0.000154062 \backslash \mathrm{H},-2.5111081041,-0.9859$ $341961,-0.887813529 \backslash \mathrm{H},-2.511119536,-0.9861299231,0.887523007 \backslash \mathrm{H},-3.3815$ $886311,0.2570464316,-0.000019952 \backslash H, 0.0000101041,-0.8811119183,0.814630$ 7742 \\Version=ES64L-G16RevB.01 \State=1-A \HF=-214.1275093 \RMSD=4.184e-0 $9 \backslash \mathrm{RMSF}=1.222 \mathrm{e}-04 \backslash$ Dipole $=0.0000003,-0.1935794,0.0000316 \backslash$ Quadrupole $=4.93$ $13237,-2.285312,-2.6460118,-0.0000005,0.0000935,-0.0000347 \backslash P G=C 01$ [X(C 4H12N1)]\\@

A2-BASE.xyz-M062X-MG3S

$1 \backslash 1 \backslash G I N C-R 841 \backslash F O p t \backslash R M 062 X \backslash G e n \backslash C 3 H 9 N 1 \backslash R O O T \backslash 17-J u n-2019 \backslash 0 \backslash \backslash \#$ M062X/Gen S

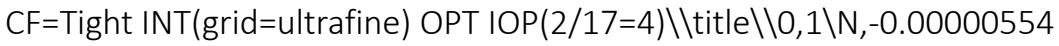
$98,0.0000266697,-0.3911408521 \backslash C, 1.3596193569,0.2234892552,0.0561257118$ $\backslash \mathrm{C},-0.8733508672,1.0657255047,0.0561550421 \backslash \mathrm{C},-0.4862769835,-1.28920676$ $06,0.0560319398 \backslash \mathrm{H}, 1.7217348591,1.1784893414,-0.3232917123 \backslash \mathrm{H}, 2.00827810$ $18,-0.5655629485,-0.3229907783 \backslash \mathrm{H}, 1.4387430461,0.2366959616,1.156286860$ $2 \backslash \mathrm{H},-0.5144883572,2.0220078985,-0.3231185648 \backslash \mathrm{H},-0.9241444566,1.1277600$ $046,1.1563161481 \backslash \mathrm{H},-1.8814917836,0.9016653833,-0.3230917468 \backslash \mathrm{H},-1.49382$ $98244,-1.4565758262,-0.323351751 \backslash \mathrm{H},-0.5146755469,-1.364267373,1.156194$ 3944\H,0.1598770052,-2.0802601107,-0.3232066912 \\Version=ES64L-G16RevB $.01 \backslash$ State $=1-A \backslash H F=-174.4392588 \backslash R M S D=9.076 e-09 \backslash R M S F=3.822 e-05 \backslash$ Dipole $=0.0$ $000046,-0.000037,0.2556463 \backslash Q u a d r u p o l e=0.9080562,0.9081363,-1.8161925,0$ $.0000224,0.0001013,0.0001795 \backslash P G=C 01[X(C 3 H 9 N 1)] \backslash \backslash @$ 
A2.xyz-M062X-MG3S

$1 \backslash 1 \backslash G I N C-R 980 \backslash F O p t \backslash R M 062 X \backslash G e n \backslash C 3 H 10 N 1(1+) \backslash R O O T \backslash 17-J u n-2019 \backslash 0 \backslash \backslash \#$ M062X/

Gen SCF=Tight INT(grid=ultrafine) OPT IOP(2/17=4)\title \1,1\N,-0.000

$0291883,0.0000649018,-0.3487783823 \backslash C, 1.2657745205,-0.6539480004,0.1011$

$30096 \backslash C,-0.0665564893,1.4232394554,0.1012925962 \backslash C,-1.199268769,-0.7692$

$796264,0.1010712302 \backslash \mathrm{H}, 2.1099012878,-0.0856032477,-0.2781235878 \backslash \mathrm{H}, 1.290$ 4937693,-1.671309527,-0.2780353919\H,1.2721015417,-0.6571674633,1.1880 $980782 \backslash \mathrm{H}, 0.8025230372,1.9531902426,-0.2772138886 \backslash \mathrm{H},-0.067640642,1.4301$ $329447,1.1882638481 \backslash \mathrm{H},-0.9804556608,1.8704141198,-0.2785004492 \backslash \mathrm{H},-2.09$ $27376581,-0.2824111673,-0.2785253103 \backslash \mathrm{H},-1.2055013641,-0.7727413099,1.1$ $880461226 \backslash \mathrm{H},-1.1287373084,-1.7846043669,-0.2777791737 \backslash \mathrm{H}, 0.0000199237,0$ $.0001110445,-1.3700837876 \backslash \backslash$ Version=ES64L-G16RevB.01 \State=1-A \HF=-174. $8103125 \backslash R M S D=6.283 e-09 \backslash R M S F=2.262 e-05 \backslash$ Dipole $=-0.0000025,0.0000301,-0.3$ 362787\Quadrupole $=0.6216156,0.6218952,-1.2435108,0.0000964,0.0000696,0$ $.0001573 \backslash P G=C 01[X(C 3 H 10 N 1)] \backslash \backslash @$

\section{A3-BASE.xyz-M062X-MG3S}

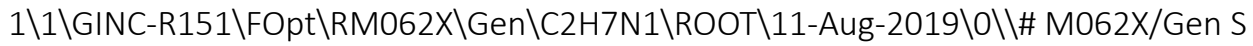

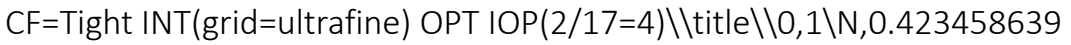

$3,-0.9838224001,-0.4444592087 \backslash C,-0.5922023186,-0.5788250521,-1.3989408$ $015 \backslash \mathrm{H}, 0.9419301528,-0.1762055705,-0.1267808692 \backslash \mathrm{H},-1.2315068882,-1.4338$ $898672,-1.6236153293 \backslash \mathrm{H},-0.1791739431,-0.2131350149,-2.3499086678 \backslash \mathrm{H},-1$. 2175854719,0.2011906669,-0.9683300007\C,1.3321656046,-1.9742505299,-0. $9921168713 \backslash \mathrm{H}, 1.8129258471,-1.6576752029,-1.9287657791 \backslash \mathrm{H}, 0.7778845756,-$ $2.8909688435,-1.198816868 \backslash \mathrm{H}, 2.1075549491,-2.2099832073,-0.2653736282 \backslash \backslash$ Version=ES64L-G16RevB.01\State $=1-A^{\prime} \backslash H F=-135.1378923 \backslash R M S D=6.958 e-09 \backslash R M S$ $\mathrm{F}=4.353 \mathrm{e}-05 \backslash \mathrm{Dipole}=0.2130908,0.2134396,-0.2758591 \backslash$ Quadrupole $=0.5389685$ $, 0.9427292,-1.4816976,0.011041,0.9531819,0.6689686 \backslash P G=C S[S G(H 1 N 1), X(C$ 2H6)]\\@

\section{A3.xyz-M062X-MG3S}

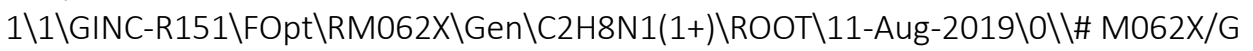

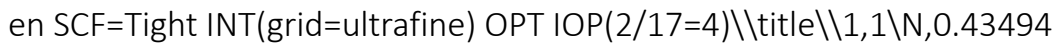
90606,-0.9691385866,-0.448453401\C, $-0.6425067492,-0.5718391528,-1.4088$ $38257 \backslash \mathrm{H}, 0.0089717218,-1.3040474033,0.4177562451 \backslash \mathrm{H}, 0.9789860091,-0.1436$ $650751,-0.1904668895 \backslash H,-1.2293263309,-1.4550455505,-1.64204561 \backslash H,-0.16$ $91033012,-0.1867507597,-2.3068318743 \backslash \mathrm{H},-1.2645101326,0.1894280599,-0.9$ 484663373\C,1.3550400505,-2.0203248594,-0.9865397549\Н,1.8229422633,-1 $.6312473429,-1.8856963804 \backslash \mathrm{H}, 0.7627192336,-2.8995421337,-1.2209101161 \backslash \mathrm{H}$ ,2.1041784795,-2.2533168636,-0.2362967125\\Version=ES64L-G16RevB.01\St ate $=1-\mathrm{A} 1 \backslash \mathrm{HF}=-135.5015915 \backslash \mathrm{RMSD}=4.716 \mathrm{e}-09 \backslash \mathrm{RMSF}=9.934 \mathrm{e}-05 \backslash \mathrm{Dipole}=0.055487$ $6,0.2305637,0.5283683 \backslash$ Quadrupole $=0.4180759,-0.1939019,-0.224174,-1.150$ 9148,0.4469593,0.1582769\PG=C02V [C2(N1),SGV(C2H2),SGV'(H2),X(H4)]\\@

\section{A4-BASE.xyz-M062X-MG3S}

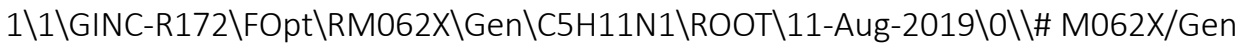
SCF=Tight INT(grid=ultrafine) OPT IOP(2/17=4)\|title \0,1\N,1.61703285 $36,-0.7867605768,2.2921469002 \backslash C, 0.9944014356,-2.104765108,2.1312158576$ $\backslash \mathrm{H}, 0.65693716,-2.5310947573,3.0867984044 \backslash \mathrm{C}, 2.0016562406,-3.0590966151$, 1.5061110021 $\backslash \mathrm{H}, 1.5133611047,-3.9904687303,1.2217482921 \backslash \mathrm{C}, 3.162123584,-$ $3.3294499208,2.4807063723 \backslash H, 2.9337531598,-4.1993325671,3.0982240272 \backslash \mathrm{C}$, $3.4236671881,-2.1091454044,3.3817266532 \backslash H, 4.4679123815,-2.0759340629,3$ $.6937840547 \backslash \mathrm{C}, 3.042812705,-0.8308595675,2.6392498631 \backslash \mathrm{H}, 3.6378370802,-0$ $.7628019235,1.723196443 \backslash H, 3.2808225849,0.053851825,3.2287591106 \backslash H, 2.82$ 
$33942116,-2.1762870862,4.2921082301 \backslash H, 4.0623693701,-3.5744869329,1.915$ $6218782 \backslash H, 2.3755470544,-2.6020835198,0.5876480169 \backslash \mathrm{H}, 0.1138559438,-2.00$ $10824413,1.4962219058 \backslash H, 1.109255942,-0.2439126113,2.9752729886|| V e r s i o$ $\mathrm{n}=\mathrm{ES64L}-\mathrm{G} 16 \mathrm{RevB} .01 \backslash \mathrm{State}=1-\mathrm{A} \backslash \mathrm{HF}=-251.8537699 \backslash \mathrm{RMSD}=6.452 \mathrm{e}-09 \backslash \mathrm{RMSF}=1.265$ e-05\Dipole $=0.0547097,-0.2135884,0.3962889 \backslash$ Quadrupole $=0.8014918,-0.574$ 042,-0.2274498, 0.5129385,-0.8815198,2.0329252\PG=C01 [X(C5H11N1)]入\@

\section{A4.xyz-M062X-MG3S}

$1 \backslash 1 \backslash G I N C-R 172 \backslash F O p t \backslash R M 062 X \backslash G e n \backslash C 5 H 12 N 1(1+) \backslash R O O T \backslash 11-A u g-2019 \backslash 0 \backslash \backslash \#$ M062X/

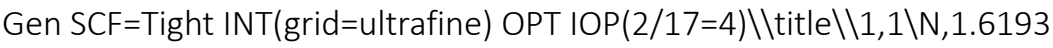
$37521,-0.7542216133,2.3391476383 \backslash C, 0.9714705277,-2.1122843581,2.113867$ $1288 \backslash \mathrm{H}, 0.6404697715,-2.46445339,3.0887325094 \backslash \mathrm{C}, 1.9885171471,-3.0543309$ $623,1.5014673903 \backslash H, 1.4806465066,-3.9767203197,1.2272968293 \backslash C, 3.1378922$ $659,-3.3265860665,2.4870726825 \backslash \mathrm{H}, 2.8989176673,-4.1870142216,3.10940579$ $59 \backslash \mathrm{C}, 3.4061452529,-2.1088180432,3.3883660553 \backslash \mathrm{H}, 4.4436242206,-2.0842997$ $917,3.7152636353 \backslash \mathrm{C}, 3.1068661097,-0.8205188878,2.6485763599 \backslash \mathrm{H}, 3.6253672$ $509,-0.7821177454,1.692684748 \backslash \mathrm{H}, 3.3523762303,0.0737876502,3.2151124674$ $\backslash \mathrm{H}, 2.8028002052,-2.1487811838,4.2980518593 \backslash \mathrm{H}, 4.0365865403,-3.581398631$ $9,1.9283726441 \backslash H, 2.3595265803,-2.6241974801,0.5684409051 \backslash H, 0.099297100$ $1,-1.9461573412,1.487234137 \backslash \mathrm{H}, 1.1370479995,-0.2800278412,3.1028586443 \backslash$ H,1.4787811033,-0.1736197723,1.5121885699||Version=ES64L-G16RevB.01\St ate $=1-A \backslash H F=-252.2292824 \backslash R M S D=4.377 \mathrm{e}-09 \backslash R M S F=5.931 \mathrm{e}-05 \backslash D i p o l e=-0.801642$ $4,1.3573388,-0.0789652 \backslash$ Quadrupole $=0.2287338,2.5142209,-2.7429547,-1.97$ $39648,0.9740046,0.2022122 \backslash P G=C 01[X(C 5 H 12 N 1)] \backslash \backslash$

A5-BASE.xyz-M062X-MG3S

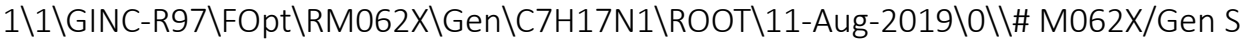

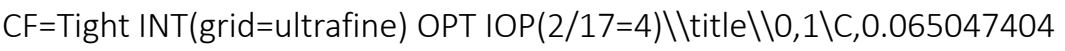
$2,0.7103639218,0.353857235 \backslash \mathrm{C}, 1.1319708385,0.1957782394,1.3131324241 \backslash \mathrm{N}$, $1.6633233246,1.2295996851,2.1899537169 \backslash \mathrm{H},-0.8843728614,0.8493204543,0$. $8800417827 \backslash \mathrm{H}, 0.3778824741,1.6939061818,-0.006512968 \backslash \mathrm{H}, 1.9684795409,-0$. $2017281266,0.731178265 \backslash \mathrm{H}, 0.7312026436,-0.6429868188,1.9097063664 \backslash \mathrm{C},-0$. $1784334583,-0.2138714515,-0.8407779526 \backslash C,-0.6012332314,-1.6144230243,-$ $0.4016402138 \backslash \mathrm{C},-1.2269130566,0.3908378547,-1.7699022438 \backslash \mathrm{H}, 0.76258658,--$ $0.2980215485,-1.3945361132 \backslash \mathrm{H},-1.5016436899,-1.5613435436,0.2156716624 \backslash$ $\mathrm{H}, 0.1742648188,-2.1131464734,0.1790228948 \backslash \mathrm{H},-0.8254456632,-2.240610448$ $2,-1.2657454748 \backslash \mathrm{H},-2.1864950259,0.4753897067,-1.2546547421 \backslash \mathrm{H},-1.377154$ $8677,-0.2303858486,-2.6533044908 \backslash \mathrm{H},-0.9357795985,1.3882737583,-2.10069$ $51985 \backslash C, 0.6383234852,1.7877659275,3.0513802441 \backslash C, 2.758437658,0.7059925$ $993,2.9826330334 \backslash H,-0.1299141415,2.2828441017,2.4610386137 \backslash \mathrm{H}, 1.0848684$ $784,2.5291197967,3.7128360877 \backslash \mathrm{H}, 0.1554717931,1.0157265997,3.6746758855$ $\backslash \mathrm{H}, 3.5402424161,0.3242792524,2.3266677753 \backslash \mathrm{H}, 2.437727094,-0.1117877087$, 3.6493507249\H,3.182457045,1.4995869129,3.5969926858\\Version=ES64L-G1 6RevB.01\State $=1-A \backslash H F=-331.6644326 \backslash R M S D=2.605 e-09 \backslash R M S F=3.005 e-06 \backslash D i p o l$ $\mathrm{e}=-0.0736058,-0.1768726,0.0915299 \backslash \mathrm{Quadrupole}=0.0289022,-1.0651989,1.03$ $62967,-1.1027604,0.22062,0.8408801 \backslash P G=C 01[X(C 7 H 17 N 1)] \backslash \backslash @$

A5.xyz-M062X-MG3S

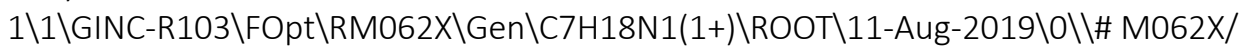

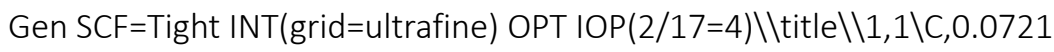
$249681,0.7340599637,0.3213111028 \backslash C, 1.1376130519,0.1974243839,1.2605158$ $971 \backslash \mathrm{N}, 1.6562493626,1.2441547142,2.2155769321 \backslash \mathrm{H},-0.8684457243,0.8848608$ $218,0.8561853804 \backslash \mathrm{H}, 0.3860273429,1.7076260417,-0.0708276384 \backslash \mathrm{H}, 2.0047516$ $421,-0.1570933149,0.7024376745 \backslash H, 0.7724882623,-0.617882439,1.884117583$ 
$1 \backslash \mathrm{H}, 2.0081795222,2.0155662728,1.6452383427 \backslash \mathrm{C},-0.1829408469,-0.21707781$ $63,-0.8561419748 \backslash C,-0.6075729295,-1.6036626528,-0.3766388356 \backslash C,-1.2394$ $313587,0.385635395,-1.7765588406 \backslash \mathrm{H}, 0.7522695181,-0.3110767638,-1.41688$ $42821 \backslash \mathrm{H},-1.4978923688,-1.5357585087,0.2529240043 \backslash \mathrm{H}, 0.1741426915,-2.113$ $6997821,0.1879466247 \backslash \mathrm{H},-0.8536961181,-2.2378028606,-1.2265226551 \backslash \mathrm{H},-2$. $1940496451,0.477528716,-1.2551067725 \backslash \mathrm{H},-1.3965690571,-0.249876828,-2.6$ 46255109\Н,-0.9482802046,1.3746682822,-2.1303426528\C,0.5838445633,1.7 $925156716,3.0970504928 \backslash \mathrm{C}, 2.794589943,0.7184844449,3.0243189486 \backslash \mathrm{H},-0.16$ $87086274,2.2794904048,2.4866967113 \backslash \mathrm{H}, 1.0280815176,2.5052918293,3.78561$ $2221 \backslash \mathrm{H}, 0.1459023168,0.9624523148,3.6462044135 \backslash \mathrm{H}, 3.5675504063,0.3566215$ $221,2.3529123492 \backslash H, 2.4214045375,-0.0960464058,3.639672682 \backslash H, 3.18118723$ 45,1.5150265933,3.6533474008\\Version=ES64L-G16RevB.01\State=1-A\HF=-3 $32.0436439 \backslash \mathrm{RMSD}=3.270 \mathrm{e}-09 \backslash \mathrm{RMSF}=1.846 \mathrm{e}-06 \backslash$ Dipole $=1.5586002,1.0886043,1$. 9812963\Quadrupole $=-0.2639611,-3.6899639,3.953925,2.8371562,7.0371787$, 5.1796072\PG=C01 [X(C7H18N1)]\\@

\section{AL1-BASE.xyz-M062X-MG3S}

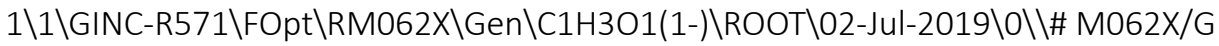
en SCF=Tight INT(grid=ultrafine) OPT IOP(2/17=4)\title \-1,1\H,1.0336 $702475,0.0011817142,-1.0202289668 \backslash C, 0.5473414796,0.0000000734,-0.00000$ $19784 \backslash \mathrm{H}, 1.0336452474,-0.8841399351,0.5091004332 \backslash \mathrm{H}, 1.0336445356,0.88295$ 90009,0.5111467719\0,-0.7868465474,-0.0000004385,-0.0000240848\|Versio $\mathrm{n}=\mathrm{ES} 64 \mathrm{~L}-\mathrm{G} 16 \mathrm{RevB} .01 \backslash \mathrm{HF}=-115.087656 \backslash \mathrm{RMSD}=2.370 \mathrm{e}-09 \backslash \mathrm{RMSF}=2.064 \mathrm{e}-04 \backslash \mathrm{Dipole}$ $=0.9799049,0.0000004,0.0000162 \backslash$ Quadrupole $=-2.2765513,1.1382756,1.13827$ $56,-0.0000013,-0.0000566,0 . \mid P G=C 03 V[C 3(C 101), 3 S G V(H 1)] \backslash \backslash @$

\section{AL1.xyz-M062X-MG3S}

$1 \backslash 1 \backslash G I N C-R 685 \backslash F O p t \backslash R M 062 X \backslash G e n \backslash C 1 H 4 O 1 \backslash R O O T \backslash 02-J u l-2019 \backslash 0 \backslash \backslash \#$ M062X/Gen S $\mathrm{CF}=$ Tight INT(grid=ultrafine) OPT IOP(2/17=4)\\title \} \backslash 0 , 1 \backslash H , 1 . 0 8 1 3 2 4 2 9 4 $9,0.9836148765,-0.0000000556 \backslash C, 0.6641073017,-0.0202706815,-0.000000055$ $6 \backslash H, 1.0239208859,-0.5426922711,-0.8896261391 \backslash H, 1.0239208859,-0.5426922$ $711,0.889626028 \backslash 0,-0.7417415841,0.1189720819,-0.0000000556 \backslash H,-1.143939$ $7843,-0.7501597346,-0.0000000556||$ Version=ES64L-G16RevB.01〈State $=1-A^{\prime} \backslash$ $\mathrm{HF}=-115.7113122 \backslash \mathrm{RMSD}=3.021 \mathrm{e}-09 \backslash \mathrm{RMSF}=4.984 \mathrm{e}-05 \backslash \mathrm{Dipole}=0.3339131,-0.5961$ $911,0 . \backslash$ Quadrupole $=0.0282138,0.5717133,-0.5999272,1.7031985,0 ., 0 . \mid \mathrm{PG}=\mathrm{CS}$ $[\mathrm{SG}(\mathrm{C} 1 \mathrm{H} 2 \mathrm{O} 1), \mathrm{X}(\mathrm{H} 2)] \backslash \backslash @$

\section{AL2-BASE.xyz-M062X-MG3S}

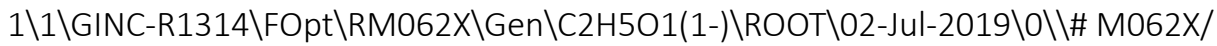
Gen SCF=Tight INT(grid=ultrafine) OPT IOP(2/17=4)\title \-1,1\H,2.009 $9139172,0.524347296,-0.00000004 \backslash C, 1.1843477584,-0.2012471465,-0.000000$ $04 \backslash \mathrm{H}, 1.2700814788,-0.8403019853,-0.8830190427 \backslash \mathrm{H}, 1.2700814788,-0.840301$ $9853,0.8830189627 \backslash \mathrm{C},-0.2101076589,0.4855946186,-0.00000004 \backslash \mathrm{H},-0.181762$ $0052,1.1884481547,0.8841168782 \backslash \mathrm{H},-0.1817620052,1.1884481547,-0.8841169$ 582\0,-1.231412964,-0.3671081069,-0.00000004\\Version=ES64L-G16RevB.01 \State $=1-A^{\prime} \backslash H F=-154.4031449 \backslash R M S D=7.191 e-09 \backslash R M S F=2.281 e-05 \backslash$ Dipole $=1.548$ 7301,0.4403152,0.\Quadrupole $=-4.0982971,1.2780525,2.8202446,-1.4884453$ ,0.,0.\PG=CS [SG(C2H1O1),X(H4)]\\@

\section{AL2.xyz-M062X-MG3S}

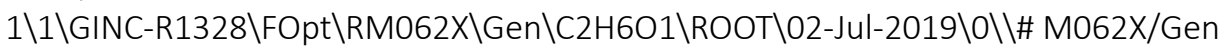
SCF=Tight INT(grid=ultrafine) OPT IOP $(2 / 17=4) \backslash$ title $\backslash \backslash 0,1 \backslash H,-2.0758249$

$534,0.4180448743,0.0554340593 \backslash C,-1.2096430721,-0.2394234276,-0.0202166$ $54 \backslash \mathrm{H},-1.2628572205,-0.9584997387,0.7993700975 \backslash \mathrm{H},-1.2669437379,-0.78686$ 
$67446,-0.9599408283 \backslash \mathrm{C}, 0.0795920741,0.5581823707,0.047008558 \backslash \mathrm{H}, 0.123682$ $2494,1.2765532698,-0.7705228669 \backslash H, 0.1293059169,1.1246830099,0.98248341$ $53 \backslash 0,1.2269770568,-0.2569797733,-0.1024090722 \backslash H, 1.2357276867,-0.912009$

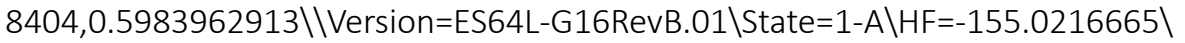
RMSD $=4.638 \mathrm{e}-09 \backslash \mathrm{RMSF}=2.801 \mathrm{e}-05 \backslash$ Dipole $=-0.5156792,-0.0597111,0.4596311 \backslash \mathrm{Q}$ uadrupole $=-1.7531979,1.1079185,0.6452794,-0.714511,1.3305792,-1.103154$ 4\PG=C01 [X(C2H6O1)]\\@

AL3-BASE.xyz-M062X-MG3S

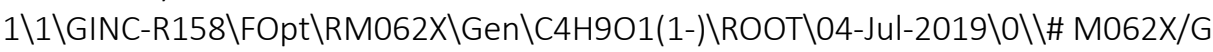
en SCF=Tight INT(grid=ultrafine) OPT IOP(2/17=4) maxdisk=5GB\\title \$1,1 \backslash \mathrm{H}, 0.0971116487,2.1558536828,0.0653499213 \backslash \mathrm{C}, 0.6505920807,1.29116207$ $99,0.438893018 \backslash \mathrm{H}, 1.6749293182,1.360493198,0.0654611859 \backslash \mathrm{H}, 0.6666885588$, $1.3232347996,1.5359316001 \backslash \mathrm{C},-0.0000009924,0.0005953399,-0.1444159785 \backslash \mathrm{C}$ $,-1.4443488773,-0.0821413632,0.4353514332 \backslash 0,0.0008877574,0.0026342485$, $-1.4798144365 \backslash C, 0.7925859007,-1.209784708,0.435163244 \backslash H, 1.818196882,-1$ $.1613484759,0.0619011355 \backslash \mathrm{H}, 0.8122370294,-1.2432849607,1.5321039368 \backslash \mathrm{H}, \mathrm{O}$ $.3406902652,-2.1304659429,0.0587586607 \backslash \mathrm{H},-1.4828753297,-0.085368801,1$. $5322941849 \backslash \mathrm{H},-1.9153374552,-0.9935170432,0.0597183549 \backslash \mathrm{H},-2.0157017866$, $0.7706299462,0.0613497398 \backslash \mid V e r s i o n=E S 64 L-G 16 R e v A .03 \backslash S t a t e=1-A \backslash H F=-233$. 0317421 \RMSD $=4.300$ e-09 $\backslash R M S F=7.392 e-06 \backslash$ Dipole $=-0.0011786,-0.0027415,1.8$ 060661 \Quadrupole $=2.0418738,2.0424215,-4.0842953,-0.0000516,0.0041178$, $0.0090624 \backslash \mathrm{PG}=\mathrm{CO} 1 \mathrm{X}(\mathrm{C} 4 \mathrm{H} 9 \mathrm{O} 1)] \backslash \backslash @$

AL3.xyz-M062X-MG3S

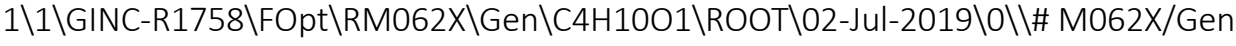
SCF=Tight INT(grid=ultrafine) OPT IOP(2/17=4)\\title \} \backslash 0 , 1 \backslash H , 0 . 2 0 2 4 9 5 4 $168,-2.1440268878,-0.1038294435 \backslash C, 0.68427462,-1.2553879787,-0.51008129$ $04 \backslash \mathrm{H}, 1.7359797578,-1.2674730769,-0.215931529 \backslash \mathrm{H}, 0.6397997812,-1.2985848$ $408,-1.5986537309 \backslash C,-0.0049347788,0.0000001341,0.0159806205 \backslash C,-1.47945$ $72583,0.0000117502,-0.3551066335 \backslash 0,0.0208378983,-0.0000000689,1.442457$ $8537 \backslash C, 0.6842943995,1.2553773878,-0.5100812904 \backslash H, 1.7359997276,1.267445$ $9156,-0.215931529 \backslash \mathrm{H}, 0.6398202412,1.2985749506,-1.5986537309 \backslash \mathrm{H}, 0.202529$ $1974,2.1440238875,-0.1038294435 \backslash \mathrm{H},-1.6021033018,0.0000127164,-1.437842$ $6379 \backslash \mathrm{H},-1.9673507801,0.8838367917,0.0542593153 \backslash \mathrm{H},-1.9673647054,-0.8838$ $056041,0.0542593153 \backslash \mathrm{H}, 0.9353426877,-0.0000072733,1.7357781583 \backslash \mid V e r s i o n$ $=E S 64 L-G 16 R e v B .01 \backslash S t a t e=1-A^{\prime} \backslash H F=-233.6447643 \backslash R M S D=5.353 e-09 \backslash R M S F=4.675$ e-05\Dipole $=0.4703627,-0.0000037,-0.4366632 \backslash$ Quadrupole $=1.1941554,-0.17$ $91472,-1.0150082,-0.0000108,2.0888483,-0.0000165 \backslash P G=C S[S G(C 2 H 2 O 1), X(C$ 2H8)]\\@

AL4-BASE.xyz-M062X-MG3S

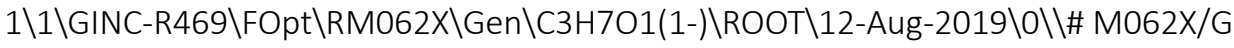
en SCF=Tight INT(grid=ultrafine) OPT IOP(2/17=4)\title \-1,1\H,-0.008 $6297477,-1.4424212409,0.3801616976 \backslash C,-0.3233303007,-0.4057876846,0.566$ $9149503 \backslash \mathrm{C}, 0.0259792997,0.0197310338,1.9871985944 \backslash \mathrm{H}, 0.200706701,0.24007$ $2812,-0.14597937 \backslash \mathrm{C},-1.8504542428,-0.2498021468,0.310661136 \backslash \mathrm{H},-2.010168$ $4267,-0.6482961388,-0.7322935643 \backslash \mathrm{H},-2.3237258821,-1.0430799293,0.96678$ $31915 \backslash 0,-2.3096324411,0.9823942936,0.5168430197 \backslash \mathrm{H},-0.3813964601,1.0181$ $967571,2.1490589517 \backslash H, 1.1022288769,0.0260365792,2.1839252891 \backslash H,-0.4483$ 073764,-0.6511843354,2.710276104\\Version=ES64L-G16RevB.01\State=1-A \H $\mathrm{F}=-193.7120904 \backslash \mathrm{RMSD}=5.882 \mathrm{e}-09 \backslash \mathrm{RMSF}=1.558 \mathrm{e}-05 \backslash \mathrm{Dipole}=1.6526659,-1.09114$ $94,0.5207459 \backslash$ Quadrupole $=-2.45326,0.0617118,2.3915482,3.7991403,-2.1086$ 45,0.6253978\PG=C01 [X(C3H7O1)]\\@ 
AL4.XYz-M062X-MG3S

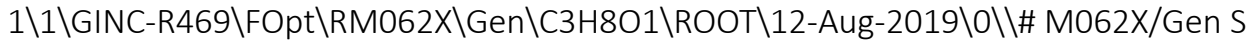

$\mathrm{CF}=$ Tight INT(grid=ultrafine) OPT IOP(2/17=4)\\title \} \backslash 0 , 1 \backslash H , 0 . 0 3 2 2 3 2 7 3 4

$1,-1.3917164678,0.3571544832 \backslash C,-0.337352539,-0.3851095253,0.5629286667$

$\backslash \mathrm{C}, 0.0000283365,0.0226082259,1.9921671743 \backslash \mathrm{H}, 0.1505075974,0.2876353782$,

$-0.1451294444 \backslash \mathrm{C},-1.8283392967,-0.3606570438,0.2925366389 \backslash \mathrm{H},-2.03181402$

$02,-0.7161368384,-0.7223351126 \backslash \mathrm{H},-2.3415321411,-1.0264851101,0.9962355$

$062 \backslash 0,-2.282278924,0.9733251056,0.4520439185 \backslash \mathrm{H},-3.2297207867,1.0068496$

$82,0.3143508421 \backslash \mathrm{H},-0.3657319856,1.0267380943,2.198191281 \backslash \mathrm{H}, 1.075438839$

$7,0.0071705267,2.1636019538 \backslash \mathrm{H},-0.4642678145,-0.6580720274,2.7077140923$

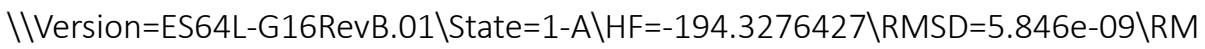

$\mathrm{SF}=1.386 \mathrm{e}-05 \backslash$ Dipole $=-0.3540623,-0.4720505,-0.1475286 \backslash$ Quadrupole $=2.5919$

$608,-1.6913571,-0.9006037,-0.1155072,0.6552954,0.1895977 \backslash P G=C 01[X(C 3 H$

801)]\\@

\section{AL5-BASE.xyz-M062X-MG3S}

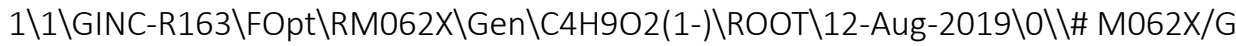
en SCF=Tight INT(grid=ultrafine) OPT IOP(2/17=4)\title \-1,1\H,0.6827

$689443,-1.5540934985,0.1079486518 \backslash C,-0.0630465456,-0.9127776942,-0.376$

$1908763 \backslash \mathrm{C}, 0.2197894126,0.5569860867,-0.1065695852 \backslash \mathrm{H},-0.0148477127,-1.0$

$885198922,-1.4581209593 \backslash \mathrm{C},-1.5077921944,-1.2655052222,0.0896696724 \backslash \mathrm{H},-$

$1.6149265511,-2.3672282514,-0.1132086448 \backslash \mathrm{H},-1.4626072966,-1.2120131034$ ,1.2179975712\0,-2.448195217,-0.498276263,-0.4621236739\С,1.4367285778 $, 1.1153389124,-0.8031822525 \backslash 0,2.6241362981,0.5042438713,-0.2870665829 \backslash$ $\mathrm{H}, 0.3166370219,0.7289776824,0.9710854348 \backslash \mathrm{H},-0.6695180062,1.099554493,-$ $0.4422225866 \backslash \mathrm{H}, 1.5001257632,2.2018175968,-0.6596873825 \backslash \mathrm{H}, 1.3577107322$, $0.9185863144,-1.8788017451 \backslash H, 3.3631467735,0.7694189679,-0.8349270411 \backslash \backslash$ Version=ES64L-G16RevB.01 \State $=1-A \backslash H F=-308.2391206 \backslash R M S D=5.334 \mathrm{e}-09 \backslash \mathrm{RMSF}$ $=7.909 \mathrm{e}-06 \backslash$ Dipole $=3.5880012,1.1681494,-0.3970117 \backslash$ Quadrupole $=-13.067146$ $9,5.8466767,7.2204702,-2.6407674,-3.5670272,-0.3277072 \backslash P G=C 01$ [X(C4H9O 2)]\\@

AL5.xyz-M062X-MG3S

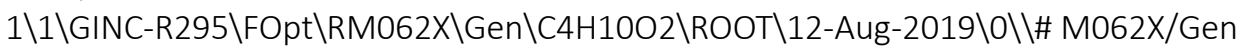
SCF=Tight INT(grid=ultrafine) OPT IOP(2/17=4)\\title $\backslash 0,1 \backslash H, 0.72768701$

09,-1.4580505188,-0.0760641844\C,-0.1057643421,-0.8615342946,-0.449107

$0073 \backslash \mathrm{C}, 0.155323215,0.6140628719,-0.1702205302 \backslash \mathrm{H},-0.1673735242,-1.02648$ $15051,-1.5283488486 \backslash \mathrm{C},-1.3883719293,-1.3517399618,0.1881673626 \backslash \mathrm{H},-1.51$ $23249021,-2.4232471645,0.004578009 \backslash \mathrm{H},-1.3451537751,-1.195561873,1.2721$ $902741 \backslash 0,-2.4687934053,-0.6230905697,-0.3743251549 \backslash \mathrm{H},-3.2901150978,-0$. $9103305065,0.0266319621 \backslash C, 1.437929747,1.1042672281,-0.8074976546 \backslash 0,2.5$ $183496538,0.3756004621,-0.2450244124 \backslash \mathrm{H}, 0.2169337891,0.7790101137,0.909$ $0211478 \backslash \mathrm{H},-0.6781288761,1.2105775619,-0.5432642148 \backslash \mathrm{H}, 1.5618919943,2.17$ $57710973,-0.6238967274 \backslash H, 1.3947021929,0.9481020701,-1.8915223772 \backslash H, 3.3$ 396882488,0.6629649888,-0.6458576438||Version=ES64L-G16RevB.01\State=1 $-A \backslash H F=-308.8535294 \backslash R M S D=2.437 e-09 \backslash R M S F=3.071 e-05 \backslash D i p o l e=0.0000134,0.00$ $0085,0.0000852 \backslash$ Quadrupole $=2.35708,-0.2290922,-2.1279878,4.1397954,-4.7$ 874794,-1.4657231\PG=C01 [X(C4H10O2)]\\@

AN1-BASE.xyz-M062X-MG3S

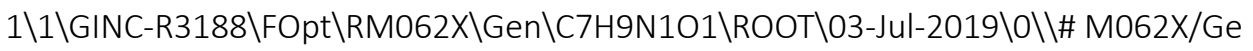
n SCF=Tight INT(grid=ultrafine) OPT IOP(2/17=4)\|title $\backslash \backslash 0,1 \backslash \mathrm{H}, 2.187104$ $1969,-2.7841151903,1.9112214951 \backslash C, 1.3920833557,-2.7058332497,1.1839920$ 
$269 \backslash \mathrm{C}, 0.0753548601,-2.8503130151,1.5763312444 \backslash \mathrm{H},-0.182318259,-3.042688$ $75,2.6089878965 \backslash C,-0.9845119082,-2.7583827994,0.679241882 \backslash 0,-2.2039702$ $278,-2.9337963439,1.284953889 \backslash C,-0.7204870746,-2.5096836734,-0.6793258$ $356 \backslash \mathrm{N},-1.6702893153,-2.3930465903,-1.6827784571 \backslash \mathrm{H},-2.651334382,-2.4756$ $424486,-1.5363784644 \backslash \mathrm{H},-1.3553182867,-2.2143551569,-2.6161765335 \backslash \mathrm{C}, 0.6$ $225293435,-2.3668739044,-1.0529685848 \backslash \mathrm{H}, 0.8318125684,-2.1750747403,-2$. $0987764186 \backslash \mathrm{C}, 1.6652635968,-2.4601748572,-0.153740082 \backslash \mathrm{H}, 2.6823585475,-2$ $.3411815425,-0.4998521432 \backslash \mathrm{C},-3.4281044536,-2.8875055555,0.597664923 \backslash \mathrm{H}$, $-4.1943165654,-3.0612903262,1.3485278856 \backslash \mathrm{H},-3.503422145,-3.676812562,-$ $0.1540537837 \backslash \mathrm{H},-3.6070762185,-1.9071449429,0.149757013 \backslash \backslash V e r s i o n=E S 64 L-$ G16RevB.01 \State $=1-A^{\prime} \backslash H F=-402.0769074 \backslash R M S D=3.433 e-09 \backslash R M S F=7.696 e-05 \backslash D i$ pole $=-0.7728837,0.0838196,-0.7519328 \backslash$ Quadrupole $=4.181028,-5.7136934,1$. 5326654,0.3160243,1.6109593,-1.1878608\PG=CS [SG(C7H7N1O1),X(H2)]\\@

AN1.xyz-M062X-MG3S

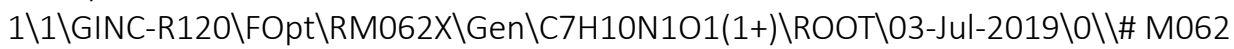

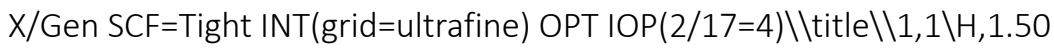
$83343103,-2.380713234,2.0601424087 \backslash C, 0.9204525914,-2.4545754881,1.1561$ $634293 \backslash \mathrm{C},-0.4595973907,-2.5695232514,1.2630691069 \backslash \mathrm{H},-0.9295588884,-2.5$ $841438869,2.2346526949 \backslash C,-1.2248786204,-2.6650616948,0.1085741365 \backslash 0,-2$ $.568053036,-2.781336783,0.0424079118 \backslash C,-0.5657334116,-2.6409127387,-1$. $1173651786 \backslash \mathrm{N},-1.4325919299,-2.7461584479,-2.3095667812 \backslash \mathrm{H},-2.4043571788$ $,-2.8197681039,-1.979797889 \backslash \mathrm{H},-1.358106018,-1.924154727,-2.9129093024 \backslash$ $\mathrm{C}, 0.8021718043,-2.5273201938,-1.2369056539 \backslash \mathrm{H}, 1.2746073434,-2.512543792$ $,-2.2112235015 \backslash \mathrm{C}, 1.5565488801,-2.4326042328,-0.0775049701 \backslash \mathrm{H}, 2.63041156$ $99,-2.3426073248,-0.1395422354 \backslash C,-3.303610334,-2.8122288346,1.26763080$ $22 \backslash \mathrm{H},-2.9987875276,-3.6690552293,1.8669723722 \backslash \mathrm{H},-4.3462241294,-2.90854$ $13033,0.987489645 \backslash H,-3.151143765,-1.8862694356,1.8205043006 \backslash H,-1.21834$ 42696,-3.574651298,-2.8689912959 \|Version=ES64L-G16RevB.01\State=1-A \H $F=-402.4485665 \backslash R M S D=6.851 e-09 \backslash R M S F=1.906 e-05 \backslash D i p o l e=-0.7752624,-0.1195$ 559,-2.0333516\Quadrupole=1.1481989,-12.494769,11.3465701,1.1987949,0. 9032889,0.7048676\PG=C01 [X(C7H10N1O1)]\\@

\section{AN2-BASE.xyz-M062X-MG3S}

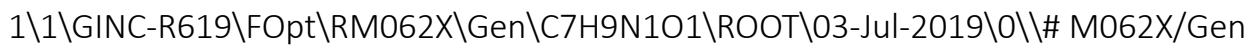
SCF=Tight INT(grid=ultrafine) OPT IOP(2/17=4)\\itle \0,1\H,2.4490090 898,-1.821155301,-2.387319584\C,1.7699558546,-1.0184383916,-2.14190085 $73 \backslash C, 0.396351773,-1.2407402954,-2.2364643868 \backslash 0,0.0244336208,-2.4824001$ $975,-2.6403077473 \backslash \mathrm{C},-0.507971847,-0.2336167314,-1.9294430597 \backslash \mathrm{H},-1.5734$ $030006,-0.3978055106,-2.0001532978 \backslash \mathrm{C},-0.0420638691,1.0223166564,-1.519$ $4293145 \backslash \mathrm{N},-0.9396226965,2.0187364327,-1.2157195141 \backslash \mathrm{H},-1.9265451836,1.8$ $644875968,-1.2819103951 \backslash \mathrm{H},-0.6210588885,2.9211403237,-0.9213953602 \backslash \mathrm{C}, 1$ .3309189159,1.2473035099,-1.4240167986\Н,1.6957882325,2.2154323655,-1. $1078625778 \backslash \mathrm{C}, 2.215297565,0.2250452704,-1.736223089 \backslash \mathrm{H}, 3.2791772023,0.40$ $6935938,-1.6598721383 \backslash \mathrm{C},-1.3504132959,-2.7595918272,-2.7524650132 \backslash \mathrm{H},-1$ $.4273690514,-3.790790764,-3.0839069688 \backslash \mathrm{H},-1.828119312,-2.1068849719,-3$ $.4874069355 \backslash \mathrm{H},-1.8570241648,-2.6501997977,-1.790307169|| V e r s i o n=E S 64 L-$

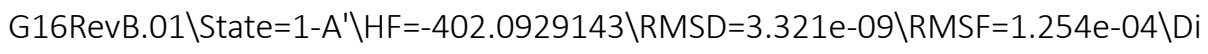
pole $=-0.9384554,0.5054675,0.1458383 \backslash$ Quadrupole $=3.2450496,2.4085281,-5$. $6535777,0.8572187,0.4419054,2.8947222 \backslash P G=C S[S G(C 7 H 7 N 1 O 1), X(H 2)] \backslash \backslash @$

AN2.xyz-M062X-MG3S

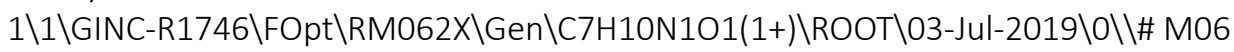
2X/Gen SCF=Tight INT(grid=ultrafine) OPT IOP $(2 / 17=4) \backslash \backslash$ title $\backslash 1,1 \backslash H, 2.3$ 
876515423,-1.754370451,-2.4196391613\C,1.7065958175,-0.9581730261,-2.1 $544715336 \backslash C, 0.329872979,-1.22420041,-2.2208951909 \backslash 0,-0.0142083948,-2.4$ $502353814,-2.6100429653 \backslash \mathrm{C},-0.5804815826,-0.2228330511,-1.8866474033 \backslash \mathrm{H}$, $-1.6472405935,-0.3924359212,-1.926452208 \backslash \mathrm{C},-0.0622973952,1.0037463862$, $-1.4997903974 \backslash \mathrm{N},-1.0359255081,2.0706135936,-1.143718789 \backslash \mathrm{H},-1.647631914$ $5,2.2976917488,-1.9320752884 \backslash \mathrm{H},-0.5445505363,2.9257711233,-0.875048794$ $7 \backslash C, 1.2749531023,1.296643942,-1.4241812794 \backslash H, 1.6323401791,2.2703606084$ $,-1.1163127984 \backslash C, 2.1676327596,0.2763118162,-1.7642864434 \backslash H, 3.230573444$ $9,0.4639408853,-1.7186873487 \backslash \mathrm{C},-1.3901536154,-2.7845180336,-2.69757566$ $16 \backslash \mathrm{H},-1.4270070294,-3.8162414261,-3.0278328446 \backslash \mathrm{H},-1.8955078557,-2.1499$ $161659,-3.4277547335 \backslash \mathrm{H},-1.8711531582,-2.6968800429,-1.7217574769 \backslash \mathrm{H},-1$. $626912241,1.7907638056,-0.3565296814 \backslash \mid$ Version=ES64L-G16RevB.01\State=1 $-A \backslash H F=-402.4403536 \backslash R M S D=4.237 e-09 \backslash R M S F=2.259 e-05 \backslash D i p o l e=-1.8820415,2.9$ $98688,0.9893637 \backslash$ Quadrupole $=1.1164043,10.7402384,-11.8566427,-4.7454176$ $,-1.7405127,8.1709378 \backslash P G=C 01[X(C 7 H 10 N 101)] \backslash \backslash @$

\section{AN3-BASE.xyz-M062X-MG3S}

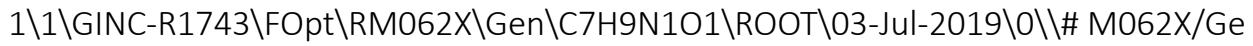

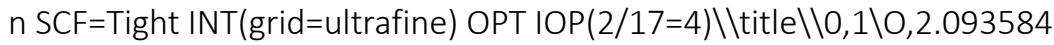
$8759,-2.9157471894,0.3764283414 \backslash C, 2.2232164028,-1.5581219631,0.3108836$ $608 \backslash C, 1.1870738817,-0.6543331995,0.4986946847 \backslash H, 0.1849056142,-0.993279$ $9233,0.7150304576 \backslash \mathrm{C}, 1.4275206373,0.7128255048,0.4100275503 \backslash H, 0.6079829$ $313,1.4047623959,0.559231982 \backslash \mathrm{C}, 2.6982358229,1.2067393759,0.1338873583 \backslash$ $\mathrm{N}, 2.9372301905,2.5637214353,0.0458106568 \backslash \mathrm{H}, 2.1989912123,3.2261910791,0$ $.1790939233 \backslash \mathrm{H}, 3.8540041413,2.9122864315,-0.1531919601 \backslash \mathrm{C}, 3.7334059111,0$ $.2837393749,-0.0531738968 \backslash \mathrm{H}, 4.7327914962,0.6406117674,-0.2694483861 \backslash \mathrm{C}$, $3.4985473599,-1.0729427401,0.0340414317 \backslash \mathrm{H}, 4.3007436191,-1.783519746,-0$ $.1110543004 \backslash \mathrm{C}, 0.8146551346,-3.4271107178,0.6547612499 \backslash \mathrm{H}, 0.9112408728,-$ $4.5088591032,0.6657729613 \backslash H, 0.0936390077,-3.1366521053,-0.1144312851 \backslash \mathrm{H}$ $, 0.4531584469,-3.0868031131,1.6291320704 \backslash \mid V e r s i o n=E S 64 L-G 16 R e v B .01 \backslash S t a$ te $=1-A^{\prime} \backslash H F=-402.0887741 \backslash R M S D=7.013 e-09 \backslash R M S F=1.528 \mathrm{e}-04 \backslash$ Dipole $=-0.325785$ $6,0.5990355,0.0500498 \backslash$ Quadrupole $=2.0086797,4.5615419,-6.5702216,3.5628$ 667,-1.9604508,-1.0653449\PG=CS [SG(C7H7N1O1),X(H2)]\\@

\section{AN3.xyz-M062X-MG3S}

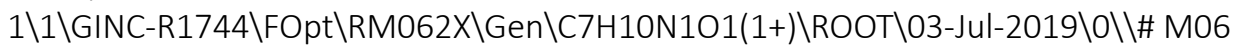

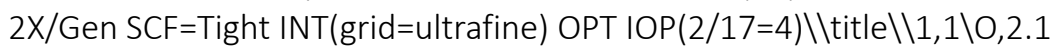
$121610449,-2.8149031459,0.4237305745 \backslash C, 2.2326912253,-1.4923561098,0.34$ $36008155 \backslash \mathrm{C}, 1.1724051478,-0.5936268203,0.4728724118 \backslash \mathrm{H}, 0.1665770141,-0.9$ $400008881,0.6505385729 \backslash \mathrm{C}, 1.4117098363,0.7695918088,0.3718141214 \backslash H, 0.58$ $77767009,1.4658824292,0.4723660982 \backslash C, 2.6962867546,1.2088622395,0.14503$ $02724 \backslash \mathrm{N}, 2.9509683524,2.6673170862,0.0371480181 \backslash \mathrm{H}, 2.0817042343,3.192461$ $0148,0.1522987488 \backslash \mathrm{H}, 3.3418870387,2.9167299505,-0.8752471111 \backslash \mathrm{C}, 3.768146$ $2302,0.3373485501,0.0126599967 \backslash \mathrm{H}, 4.7724494129,0.7016675516,-0.16560261$ $27 \backslash C, 3.5317428967,-1.013378165,0.1126916837 \backslash \mathrm{H}, 4.3345610469,-1.72991799$ $71,0.0164497226 \backslash \mathrm{C}, 0.8271039632,-3.3770060362,0.6559756989 \backslash \mathrm{H}, 0.97369502$ $75,-4.4506082418,0.6815011351 \backslash \mathrm{H}, 0.1427475926,-3.1187691244,-0.15301581$ $92 \backslash H, 0.4264455157,-3.0380716299,1.6120589248 \backslash H, 3.603310965,2.991787527$

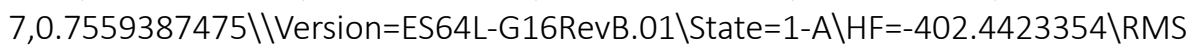
$\mathrm{D}=8.577 \mathrm{e}-09 \backslash \mathrm{RMSF}=7.726 \mathrm{e}-06 \backslash \mathrm{Dipole}=0.3678355,3.8374732,-0.2353717 \backslash \mathrm{Quadr}$ upole $=-5.679157,22.0130852,-16.3339283,9.9979792,-2.2556947,-3.3909236$ $\backslash \mathrm{PG}=\mathrm{CO} 1[\mathrm{X}(\mathrm{C} 7 \mathrm{H} 10 \mathrm{~N} 1 \mathrm{O} 1)] \backslash \backslash @$

AN4-BASE.xyz-M062X-MG3S 


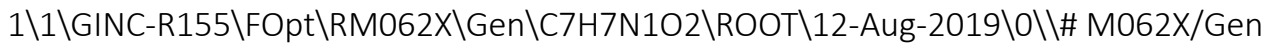

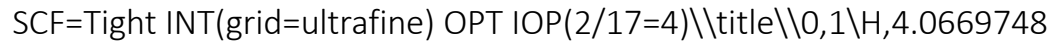
$272,0.8684666527,-1.3121423045 \backslash C, 3.1556354278,0.2938359859,-1.21049635$ 03\C,2.1919252968,0.7011433408,-0.2979403024\Н,2.3735277058,1.56529844 $64,0.3261201067 \backslash \mathrm{C}, 0.9943472855,0.0194300032,-0.1828958084 \backslash \mathrm{H}, 0.25394754$ $07,0.341058001,0.538633982 \backslash C, 0.7247704108,-1.0861705711,-0.9959618797 \backslash$ $\mathrm{N},-0.4542253135,-1.7994477072,-0.8513605183 \backslash \mathrm{H},-1.2267842998,-1.3051479$ 793,-0.4374510349\Н,-0.7242403117,-2.3721706658,-1.6336953933\C,1.6894 704949,-1.4874605052,-1.9177110724\Н,1.5144003645,-2.3330520394,-2.570 $2009518 \backslash C, 2.8957552904,-0.8108458472,-2.0165683847 \backslash C, 3.8708681253,-1.3$ $136979522,-3.0385343969 \backslash 0,3.5520077548,-1.9822137201,-3.9759578066 \backslash 0,5$ $.162488382,-0.9772295496,-2.8535851784 \backslash H, 5.2837810186,-0.554255893,-1$. 999002706||Version=ES64L-G16RevB.01 \State $=1-A \backslash H F=-476.1503757 \backslash$ RMSD $=4.4$ 93e-09\RMSF $=1.731$ e-05\Dipole $=-1.1050277,0.8208835,1.4440801 \backslash$ Quadrupole $=7.1427084,-0.2967533,-6.8459551,4.3800289,3.6158379,0.7034071 \backslash P G=C 01$ [X(C7H7N1O2)]\\@

\section{AN4.xyz-M062X-MG3S}

$1 \backslash 1 \backslash G I N C-R 163 \backslash F O p t \backslash R M 062 X \backslash G e n \backslash C 7 H 8 N 102(1+) \backslash R O O T \backslash 12-A u g-2019 \backslash 0 \backslash \backslash \#$ M062X

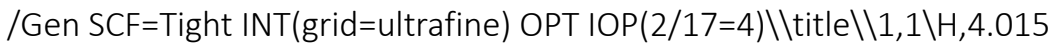
$2813418,0.8292868342,-1.1448116882 \backslash C, 3.0690274916,0.3091534978,-1.1048$ $56954 \backslash C, 2.0562916029,0.7549002352,-0.2661103069 \backslash \mathrm{H}, 2.206865647,1.626467$ $611,0.3539421898 \backslash \mathrm{C}, 0.8437533293,0.0825473204,-0.2188395426 \backslash \mathrm{H}, 0.0502405$ $875,0.4256268058,0.4336502069 \backslash \mathrm{C}, 0.684892609,-1.0291109532,-1.025016140$ $4 \backslash \mathrm{N},-0.6100742582,-1.7603108901,-0.9892318764 \backslash \mathrm{H},-1.2439992255,-1.34837$ 44184,-0.3008499224\Н,-0.4747307979,-2.7433552951,-0.7375326802\C,1.66 $67395439,-1.4984258571,-1.867697707 \backslash \mathrm{H}, 1.5413800476,-2.3704664976,-2.49$ $95401163 \backslash C, 2.8732893515,-0.812662043,-1.9012316656 \backslash C, 3.9237246914,-1.3$ $476944905,-2.8279647384 \backslash 0,3.7496691729,-2.3145471753,-3.5108684022 \backslash 0,5$ $.0427651369,-0.6289047528,-2.7937981792 \backslash \mathrm{H}, 5.6820031221,-1.0173526436,-$ 3.4076288829\H,-1.0789293938,-1.73308872876,-1.8990535942\|Version=ES64

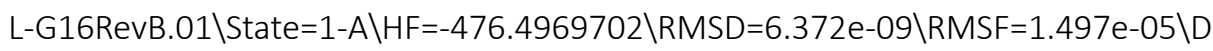
ipole $=-3.8272656,-0.4082146,1.50118 \backslash$ Quadrupole $=21.0642648,-9.9870196$,$11.0772452,9.7037081,-9.9150941,-0.008876 \backslash P G=C 01[X(C 7 H 8 N 102)] \backslash \backslash @$

\section{AN5-BASE.xyz-M062X-MG3S}

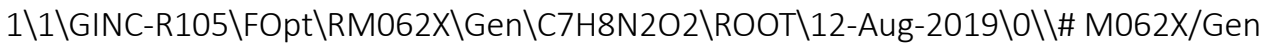

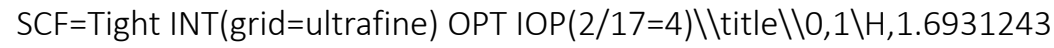
739,-3.2691678344,1.1746224901 \C,1.1993591393,-2.7780910991,0.35196426 $48 \backslash C,-0.1685937206,-2.885696418,0.1874884827 \backslash \mathrm{N},-0.9433202175,-3.683959$ $627,1.1563748252 \backslash \mathrm{C},-0.847464398,-2.2786512453,-0.8454394983 \backslash \mathrm{H},-1.91820$ $62551,-2.3996708815,-0.9211830591 \backslash \mathrm{C},-0.1436962494,-1.5151539311,-1.780$ $8207158 \backslash \mathrm{N},-0.8388817141,-0.9177202661,-2.8037144564 \backslash \mathrm{H},-1.8316967447,-1$ $.0329063871,-2.8696616527 \backslash \mathrm{H},-0.3885325195,-0.3609444854,-3.5005179855 \backslash$ C,1.2506536548,-1.3837112842,-1.6445153887\C,2.0905491418,-0.581643901 $3,-2.6036617614 \backslash \mathrm{C}, 1.8831910949,-2.0192839376,-0.5813171502 \backslash \mathrm{H}, 2.9567416$ $756,-1.9107785951,-0.4868921066 \backslash 0,-2.140980053,-3.7638539646,0.9917542$ $85 \backslash 0,-0.3344565273,-4.2121166029,2.0593232281 \backslash \mathrm{H}, 1.5228711899,-0.117300$ $916,-3.4050742894 \backslash \mathrm{H}, 2.6135145354,0.2159562852,-2.0755417102 \backslash \mathrm{H}, 2.845591$ $615,-1.2154242712,-3.0692828036||$ Version=ES64L-G16RevB.01\State $=1-A^{\prime} \backslash \mathrm{H}$ $\mathrm{F}=-531.3855492 \backslash \mathrm{RMSD}=6.370 \mathrm{e}-09 \backslash \mathrm{RMSF}=1.451 \mathrm{e}-05 \backslash \mathrm{Dipole}=0.7806583,1.450483$ ,$-1.9069557 \backslash$ Quadrupole $=2.5389314,-2.0669424,-0.471989,-2.372798,4.4411$ 769,-0.2319297\PG=CS [SG(C7H6N2O2),X(H2)]入\@ 
AN5.xyz-M062X-MG3S

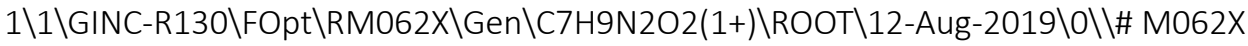

/Gen SCF=Tight INT(grid=ultrafine) OPT IOP(2/17=4)\title \\1,1\H,1.613

7597246,-3.0828210438,1.2802591699\C,1.1425493987,-2.6475142667,0.4107

$324511 \backslash \mathrm{C},-0.2019604719,-2.8623018175,0.1891842683 \backslash \mathrm{N},-0.9824266186,-3.6$

$845588858,1.1423877487 \backslash \mathrm{C},-0.8633151594,-2.3343666169,-0.902409585 \backslash \mathrm{H},-1$ $.9198865956,-2.531141223,-1.033273911 \backslash \mathrm{C},-0.1124963875,-1.5748290753,-1$ $.7705068272 \backslash \mathrm{N},-0.7952768131,-0.9931762931,-2.9539360508 \backslash \mathrm{H},-1.784989204$ $,-1.2509780398,-2.9645733648 \backslash \mathrm{H},-0.3765073422,-1.3222927791,-3.82932398$ $54 \backslash C, 1.2459012247,-1.3152008454,-1.6128467574 \backslash C, 2.0046114057,-0.476063$ $9506,-2.6011044227 \backslash C, 1.8560948852,-1.874023099,-0.4945423869 \backslash \mathrm{H}, 2.91053$ $52763,-1.6988148819,-0.3314251782 \backslash 0,-2.1543571705,-3.8351697369,0.8906$ $377983 \backslash 0,-0.3861375608,-4.1330490509,2.0857701825 \backslash \mathrm{H}, 1.6017394256,0.537$ $765415,-2.6663731612 \backslash \mathrm{H}, 3.0462460671,-0.3882803801,-2.3064621188 \backslash \mathrm{H}, 1.98$ $89710901,-0.9144470349,-3.602130791 \backslash \mathrm{H},-0.7382051743,0.0299236059,-2.95$ 60530784\|Version=ES64L-G16RevB.01 \State $=1-A \backslash H F=-531.7179865 \backslash R M S D=7.74$ 7e-09 \RMSF $=1.147$ e-05\Dipole $=0.2761819,2.9016065,-4.3854363 \backslash$ Quadrupole $=$ $-1.7955867,-5.3369964,7.1325832,-2.9371571,8.1361698,-9.2032473 \backslash P G=C 01$ [X(C7H9N2O2)]\\@

C1-BASE.xyz-M062X-MG3S

$1 \backslash 1 \backslash G I N C-R 1175 \backslash F O p t \backslash R M 062 X \backslash G e n \backslash C 8 H 7 O 2(1-) \backslash R O O T \backslash 11-J u n-2019 \backslash 0 \backslash \backslash \#$ M062X/ Gen SCF=Tight INT(grid=ultrafine) OPT IOP(2/17=4)\title \-1,1\0,2.646 $7405688,-1.3525528702,0.4220681744 \backslash 0,3.5552306653,0.6821974357,0.76535$ $24569 \backslash C, 1.6569432281,0.5238378549,-0.6719830618 \backslash C, 2.7351737331,-0.1183$ $798991,0.2888879268 \backslash C, 0.2429752261,0.211117356,-0.2938944184 \backslash C,-1.5357$ $077821,-1.3929718603,0.0700962609 \backslash C,-2.4096884167,-0.3752615241,0.4408$ $402355 \backslash C,-0.6422301234,1.2190920986,0.0876216198 \backslash C,-1.9534992665,0.934$ $6361782,0.4509886131 \backslash \mathrm{C},-0.2287475238,-1.1061307085,-0.2905262219 \backslash \mathrm{H},-0$. $2903209836,2.2438141453,0.1030190227 \backslash \mathrm{H},-2.6173661463,1.7388595737,0.74$ $4096814 \backslash \mathrm{H},-3.4298522714,-0.6032238116,0.7225602992 \backslash H, 1.8122074689,1.60$ $03896616,-0.7068483148 \backslash \mathrm{H}, 1.866442734,0.1093363469,-1.6614939935 \backslash \mathrm{H}, 0.46$ $87165049,-1.8937060469,-0.5407075105 \backslash H,-1.8770176154,-2.4210539303,0.0$

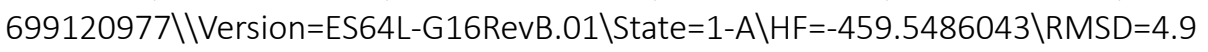
10e-09\RMSF $=2.471$ e-05\Dipole $=-3.7501665,0.4339622,-0.9731447 \backslash$ Quadrupol $\mathrm{e}=-16.4249255,8.0302261,8.3946995,0.8172815,-7.3076822,0.4889319 \backslash \mathrm{PG}=\mathrm{CO}$ $1[X(\mathrm{C} 8 \mathrm{H} 7 \mathrm{O} 2)] \backslash \backslash @$

C1.xyz-M062X-MG3S

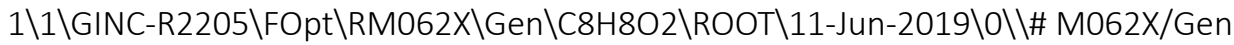

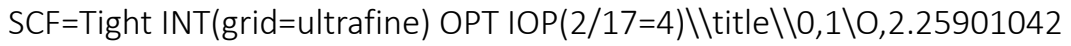
$12,-1.1194711033,0.8597044615 \backslash 0,2.6479893204,1.0414087046,0.4458796408$ $\backslash C, 1.1772095973,-0.1770801262,-1.0207803235 \backslash C, 2.1141206508,0.011544530$ $6,0.1468937367 \backslash C,-0.2526098217,-0.0434677669,-0.5436195 \backslash C,-2.078475450$ $6,1.3516593633,0.1871150056 \backslash C,-2.8675056479,0.2258865026,0.3842002841 \backslash$ C,-1.0449823524,-1.1666818842,-0.3383605849\C,-2.3480418603,-1.0333997 $87,0.1213229715 \backslash \mathrm{C},-0.7774619154,1.2170538839,-0.2731626696 \backslash \mathrm{H},-0.638468$ $907,-2.1500141343,-0.5385524931 \backslash H,-2.9569359981,-1.9141895214,0.273367$ $0437 \backslash \mathrm{H},-3.883103788,0.3305088008,0.7404535291 \backslash \mathrm{H}, 2.8203290898,-0.914744$ $0356,1.6185945375 \backslash H, 1.4109842939,0.593091665,-1.7519264142 \backslash H, 1.3394585$ $75,-1.1597257777,-1.4590480091 \backslash \mathrm{H},-0.157654307,2.0929306702,-0.42094321$

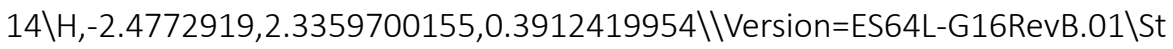
ate $=1-A \backslash H F=-460.1024863 \backslash R M S D=8.188 \mathrm{e}-09 \backslash R M S F=1.677 \mathrm{e}-06 \backslash$ Dipole $=-0.367520$ $5,-0.548241,-0.0575181 \backslash$ Quadrupole $=0.1695906,-0.2203805,0.0507898,-3.88$ 
39797,-0.8791451,-0.7006964\PG=C01 [X(C8H8O2)]\\@

C2-BASE.xyz-M062X-MG3S

$1 \backslash 1 \backslash G I N C-R 2205 \backslash F O p t \backslash R M 062 X \backslash G e n \backslash C 3 H 5 O 3(1-) \backslash R O O T \backslash 11-J u n-2019 \backslash 0 \backslash \backslash \#$ M062X/

Gen SCF=Tight INT(grid=ultrafine) OPT IOP(2/17=4)\title \-1,1\0,1.944

$1211552,-0.9404421309,-0.2495032318 \backslash 0,1.8261009944,1.22059567,0.391787$

$603 \backslash C,-0.1387667861,0.2039074929,-0.4460954699 \backslash C, 1.3714710518,0.143915$ $2408,-0.0644509402 \backslash H,-0.211321438,-0.0282163837,-1.5152876423 \backslash 0,-0.636$ $3714603,1.5050162154,-0.2114767038 \backslash C,-0.9358469397,-0.8260082965,0.339$ $9415657 \backslash \mathrm{H},-0.8537221505,-0.6129890611,1.4073808837 \backslash \mathrm{H},-0.537955341,-1.8$ $217479415,0.1497901032 \backslash H,-1.9918049282,-0.791777342,0.0634170658 \backslash H, 0.1$ $640958425,1.9477465367,0.1345067665 \backslash \backslash$ Version=ES64L-G16RevB.01\State $=1-$ $\mathrm{A} \backslash \mathrm{HF}=-343.0636939 \backslash \mathrm{RMSD}=3.658 \mathrm{e}-09 \backslash \mathrm{RMSF}=1.741 \mathrm{e}-05 \backslash \mathrm{Dipole}=-1.6063944,-0.1$ 999271,-0.1193362\Quadrupole $=-5.4706746,-0.461872,5.9325465,3.1673729$, $-0.8192272,-0.7544101 \backslash P G=C 01[X(C 3 H 5 O 3)] \backslash \backslash @$

\section{C2.xyz-M062X-MG3S}

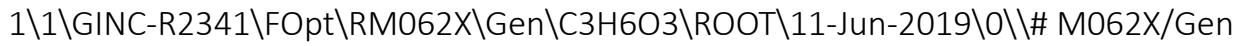

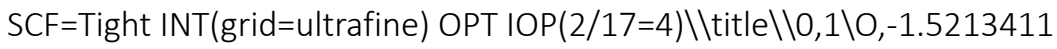

$655,0.9241723491,-0.2456591989 \backslash 0,-1.1509968346,-1.2217111887,0.2628438$ $566 \backslash C, 0.6993318757,0.1093058491,-0.4805350377 \backslash C,-0.7444987339,-0.15548$ $90133,-0.1101303484 \backslash H, 0.6964146367,0.4430430406,-1.5245088678 \backslash 0,1.4396$ $881051,-1.0724780273,-0.3625195255 \backslash C, 1.2961869119,1.2053805657,0.39432$ $90761 \backslash \mathrm{H},-2.4213619606,0.6764116268,0.0029816526 \backslash \mathrm{H}, 1.2447289618,0.91444$ $8828,1.4429554831 \backslash \mathrm{H}, 0.7691894971,2.146757608,0.2539263278 \backslash \mathrm{H}, 2.34165915$ $81,1.3353132086,0.124080419 \backslash H, 0.8366595483,-1.7765248467,-0.0959238371$

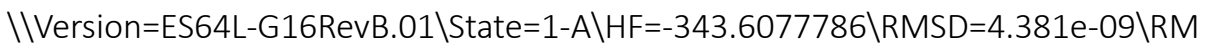
$S F=1.479 e-05 \backslash$ Dipole $=-0.5408302,0.7492873,-0.0244872 \backslash$ Quadrupole $=1.06317$ $19,-1.3647104,0.3015385,-0.1244461,0.4447091,0.41938 \backslash P G=C 01[X(C 3 H 6 O 3)$ ]\@

\section{C3-BASE.xyz-M062X-MG3S}

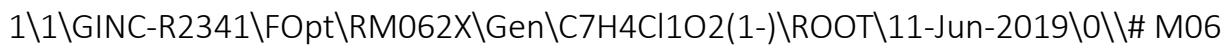
2X/Gen SCF=Tight INT(grid=ultrafine) OPT IOP(2/17=4)\\title \\-1,1\0,-0 $.098773536,2.1807684299,0.1517858281 \backslash 0,-1.8585308462,0.7841604499,0.36$ $11487941 \backslash C, 0.3108459525,-0.142052321,0.2583335086 \backslash C,-0.6534250173,1.07$ $2741481,0.2569379889 \backslash C,-0.1803928491,-1.4376741976,0.3698937131 \backslash C, 1.68$ $42686005,0.0421895573,0.1480500251 \backslash C, 2.5580062031,-1.0377495122,0.1480$ $947315 \backslash C, 0.6730857895,-2.5336915243,0.3723486819 \backslash C, 2.0364328797,-2.315$ $9560985,0.2608308259 \backslash \mathrm{H}, 2.0445407613,1.0590261592,0.0623247103 \backslash \mathrm{H},-1.252$ $5539741,-1.5576692181,0.4545887624 \backslash \mathrm{H}, 0.2957353974,-3.5436398803,0.4589$ $070509 \backslash \mathrm{Cl}, 3.1264408038,-3.689153998,0.2624083156 \backslash \mathrm{H}, 3.6273354225,-0.899$ $5598389,0.0625378136 \backslash \mid$ Version=ES64L-G16RevB.01 \State $=1-A 1 \backslash H F=-879.8612$ $63 \backslash$ RMSD $=6.679 \mathrm{e}-10 \backslash \mathrm{RMSF}=3.195 \mathrm{e}-05 \backslash \mathrm{Dipole}=2.5988509,-3.2740465,0.0037611$ Vuadrupole $=-2.5980134,-11.7230425,14.3210559,19.5807736,0.0780746,0.1$ 097474\PG=C02V [C2(C1C1C1Cl1),SGV(C4H4O2)]\\@

\section{C3.xyz-M062X-MG3S}

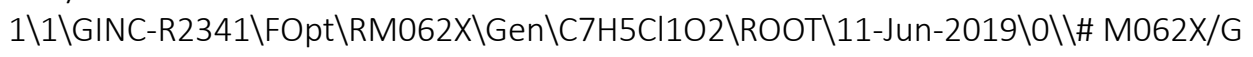

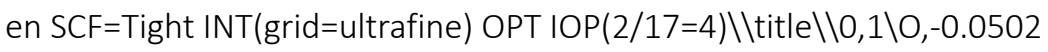
$553378,2.1923114184,0.1473912902 \backslash 0,-1.838568163,0.8725038176,0.3545083$ $892 \backslash C, 0.3153777208,-0.1381056424,0.2576427705 \backslash C,-0.6480021181,0.992288$ $7564,0.2611479226 \backslash C,-0.1928390904,-1.4281101916,0.3704291556 \backslash C, 1.68709$ $25457,0.063761876,0.1461037665 \backslash C, 2.5504701058,-1.019259262,0.147047872$ 
$5 \backslash C, 0.6613228682,-2.5159435351,0.372340873 \backslash C, 2.0278411699,-2.298893117$ $5,0.2602780808 \backslash \mathrm{H}, 2.0767661317,1.0674445941,0.0587716082 \backslash \mathrm{H},-1.262251121$ $8,-1.5619140228,0.4559895794 \backslash \mathrm{H}, 0.2828144917,-3.5238480785,0.4591225533$ $\backslash \mathrm{Cl}, 3.102924025,-3.6551542414,0.2619175404 \backslash \mathrm{H}, 3.6182367967,-0.880719608$ $9,0.0613051857 \backslash \mathrm{H},-0.7488000243,2.8584472378,0.1589134122 \backslash \backslash V e r s i o n=E S 64$ L-G16RevB.01 \State $=1-A \backslash H F=-880.4063605 \backslash R M S D=9.016 \mathrm{e}-09 \backslash \mathrm{RMSF}=4.470 \mathrm{e}-06 \backslash \mathrm{D}$ ipole $=0.3874423,0.3942814,-0.0512574 \backslash$ Quadrupole $=-3.7169258,5.4925751,-$ $1.7756492,2.5455061,-0.0123348,-0.6143742 \backslash P G=C 01[X(C 7 H 5 C l 102)] \backslash \backslash @$

C4-BASE.xyz-M062X-MG3S

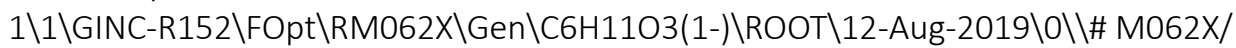
Gen SCF=Tight INT(grid=ultrafine) OPT IOP(2/17=4)\title \-1,1\0,0.713 $1965927,2.8828046691,1.2648619757 \backslash C, 0.0474005331,3.2943658597,2.442545$ $4366 \backslash \mathrm{H},-0.94050493,2.8183339716,2.5035425444 \backslash \mathrm{H}, 0.6415524975,2.92608709$ $15,3.278629513 \backslash C,-0.1470579644,4.8283025608,2.6446187141 \backslash 0,-0.75878646$ $56,5.0962946154,3.6966234023 \backslash 0,0.3147246636,5.584440358,1.771938631 \backslash C$, $-0.0328518216,3.1305769156,0.1047341863 \backslash C, 0.841685042,2.8565710814,-1$. $1028028098 \backslash C, 0.1018495522,3.0687119449,-2.4176107925 \backslash C, 0.9829980476,2$. $8083408072,-3.6348199665 \backslash \mathrm{H},-0.3718713656,4.1700364999,0.0956527633 \backslash \mathrm{H},-$ $0.9196795152,2.4734877079,0.0826734932 \backslash H, 1.2216400087,1.8315885168,-1$. $0491159159 \backslash \mathrm{H}, 1.707595347,3.5210587009,-1.0503574815 \backslash \mathrm{H},-0.279124276,4.0$ $923391305,-2.4520030654 \backslash \mathrm{H},-0.7735381025,2.4137138314,-2.4528368195 \backslash \mathrm{H}, 1$ $.8496628725,3.4712936452,-3.6299831263 \backslash \mathrm{H}, 0.4442833736,2.9663422319,-4$. $5701379921 \backslash H, 1.3552459101,1.7821398603,-3.6303826902 \backslash \backslash V e r s i o n=E S 64 L-G 1$ 6RevB.01 \State $=1-A \backslash H F=-460.9533606 \backslash R M S D=5.506 e-09 \backslash R M S F=3.489 e-06 \backslash D i p o l$ e=0.4188362,-2.5836761,-3.85603\Quadrupole $=10.7299159,0.7607105,-11.49$ 06263,3.3354806,6.7814182,-13.5363159\PG=C01 [X(C6H11O3)]\\@

\section{C4.xyz-M062X-MG3S}

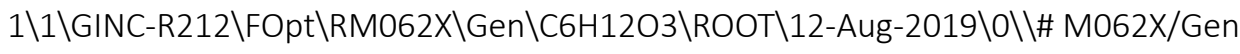
SCF=Tight INT(grid=ultrafine) OPT IOP(2/17=4)\|title \0,1\0,0.73309172

$19,3.041032259,1.2722713616 \backslash C, 0.0222232983,3.2871193305,2.439853542 \backslash \mathrm{H}$, $-0.9375018953,2.7599816919,2.4662959999 \backslash \mathrm{H}, 0.6190344579,2.9271573533,3$. $2794033392 \backslash C,-0.2746250251,4.7503343421,2.7272554058 \backslash 0,-1.0399478958,5$ $.1067597478,3.5773375181 \backslash 0,0.428448251,5.5987021529,1.9655318249 \backslash H, 0.1$ $917980924,6.4930401352,2.2446550093 \backslash C,-0.0446700562,3.1750690838,0.098$ $1236812 \backslash C, 0.8428955992,2.9344556929,-1.1024382285 \backslash C, 0.0761233937,3.042$ 7238816,-2.4154217217\C,0.9698798431,2.8015405983,-3.6261441312\H,-0.4 $826136601,4.1789927716,0.0494449001 \backslash \mathrm{H},-0.8680259921,2.4490115319,0.121$ $3658568 \backslash \mathrm{H}, 1.2954405901,1.9441389701,-1.0130835167 \backslash \mathrm{H}, 1.6591546586,3.659$ 9611074,-1.0832621171 \H,-0.3812980108,4.0327393064,-2.4879672107\H,-0. $746098652,2.322495916,-2.4167758627 \backslash \mathrm{H}, 1.7828256241,3.52781161,-3.65777$ $70785 \backslash \mathrm{H}, 0.4119253585,2.8813057597,-4.5581194844 \backslash \mathrm{H}, 1.4161902984,1.80725$ 67576,-3.5857290871\|Version=ES64L-G16RevB.01\State=1-A\HF=-461.505595 $6 \backslash \mathrm{RMSD}=7.651 \mathrm{e}-09 \backslash \mathrm{RMSF}=1.356 \mathrm{e}-06 \backslash \mathrm{Dipole}=0.022199,-0.0015991,-0.671366 \backslash \mathrm{Q}$ uadrupole $=-1.9370499,4.6866107,-2.7495608,2.1768668,3.7285822,-1.49645$ $27 \backslash P G=C 01[X(\mathrm{C} 6 \mathrm{H} 12 \mathrm{O} 3)] \backslash \backslash @$

C5-BASE.xyz-M062X-MG3S

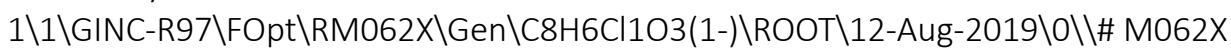
/Gen SCF=Tight INT(grid=ultrafine) OPT IOP(2/17=4)\|title \-1,1\0,3.26 19437156,-0.395184458,1.1701590033\C,1.974651851,-0.6858215818,1.71229 04533\H,1.5691097468,-1.5805782655,1.2330984046\H,2.1582270753,-0.9123 $070759,2.7598592988 \backslash C, 0.9195971577,0.4592881329,1.6353608932 \backslash 0,-0.1948$ 
$510383,0.092009011,2.0506862158 \backslash 0,1.306657362,1.5453108016,1.185195538$ $3 \backslash C, 3.3776048375,-0.2428330377,-0.1466449907 \backslash C, 2.3154827876,-0.2841737$ $641,-1.0555823096 \backslash \mathrm{H}, 1.3063153726,-0.3752668523,-0.6852689138 \backslash \mathrm{C}, 2.54616$ $11801,-0.1541391484,-2.4151060174 \backslash \mathrm{H}, 1.7021835788,-0.1771807288,-3.0918$ $397821 \backslash \mathrm{C}, 3.8297255972,0.0237197243,-2.9122434638 \backslash \mathrm{H}, 4.0048243893,0.1306$ $143431,-3.9736995009 \backslash \mathrm{C}, 4.8938088972,0.0723358297,-2.0231369428 \backslash \mathrm{H}, 5.908$ $2763838,0.2118891625,-2.3702973316 \backslash C, 4.6673939434,-0.0604546711,-0.666$ $0839581 \backslash \mathrm{Cl}, 6.0177071621,-0.0084774215,0.4267134036 \mid \backslash V e r s i o n=E S 64 L-G 16 R$ evB.01 \State $=1-A \backslash H F=-994.3769706 \backslash R M S D=7.704 \mathrm{e}-09 \backslash R M S F=3.606 \mathrm{e}-06 \backslash \mathrm{Dipole}=$ 3.049827,-1.4168238,-2.4384005\Quadrupole $=-14.2355946,7.2740733,6.9615$ 213,6.5053881,14.3899693,-3.8535603\PG=C01 [X(C8H6Cl1O3)]\\@

\section{C5.xyz-M062X-MG3S}

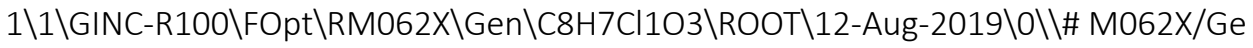
n SCF=Tight INT(grid=ultrafine) OPT IOP(2/17=4)\\title \0,1\0,3.217289 7573,-0.4600658319,1.2096156584\C,1.9504537808,-0.7446314658,1.7282231 $103 \backslash \mathrm{H}, 1.4907004964,-1.6137925577,1.2551468678 \backslash \mathrm{H}, 2.0984966471,-0.969125$ $3087,2.7840985394 \backslash C, 0.9596624734,0.4045399979,1.6496709753 \backslash 0,-0.225692$ $526,0.2460994403,1.7135362687 \backslash 0,1.552858502,1.5946261977,1.5424394332 \backslash$ $\mathrm{H}, 0.8596142322,2.2671456884,1.5109640184 \backslash \mathrm{C}, 3.3333532953,-0.2881066374$, $-0.1303506624 \backslash C, 2.2754808809,-0.3690167326,-1.0287825951 \backslash \mathrm{H}, 1.272407086$ $7,-0.5763553875,-0.6837421167 \backslash C, 2.4974695212,-0.1818162179,-2.38773980$ $41 \backslash \mathrm{H}, 1.6624982338,-0.2481361224,-3.0710160856 \backslash \mathrm{C}, 3.7684782443,0.0877829$ $073,-2.8627279604 \backslash \mathrm{H}, 3.9418377863,0.2340941988,-3.9189712941 \backslash \mathrm{C}, 4.829080$ $2551,0.1710585118,-1.9698956268 \backslash \mathrm{H}, 5.8322169013,0.3802716653,-2.3132863$ $401 \backslash C, 4.6147709718,-0.0145974423,-0.6178314796 \backslash C l, 5.9374334603,0.08695$ $50969,0.4877990935 \backslash \backslash$ Version=ES64L-G16RevB.01\State=1-A\HF=-994.9166522 $\backslash$ RMSD $=6.092 \mathrm{e}-09 \backslash \mathrm{RMSF}=9.170 \mathrm{e}-06 \backslash \mathrm{Dipole}=-0.3010035,0.1460339,-0.4145814 \backslash$ Quadrupole $=-6.665755,1.6208363,5.0449187,-1.3088063,-0.1260408,-0.4288$ 597\PG=C01 [X(C8H7Cl1O3)]\\@

\section{CA1-BASE.xyz-M062X-MG3S}

$1 \backslash 1 \backslash G I N C-R 193 \backslash F O p t \backslash R M 062 X \backslash G e n \backslash C 3 H 7 N 1 O 2 \backslash R O O T \backslash 02-J u l-2019 \backslash 0 \backslash \backslash \#$ M062X/Gen SCF=Tight INT(grid=ultrafine) OPT IOP(2/17=4)\\title $\backslash(0,1 \backslash \mathrm{H},-1.563197$ $1075,3.8777964316,0.5014622669 \backslash \mathrm{C},-1.498729101,2.8482776312,0.215897613$ $7 \backslash \mathrm{N},-0.3730471343,2.0211322761,0.6605728803 \backslash \mathrm{H}, 0.540887187,2.3095880173$ $, 0.3141627206 \backslash \mathrm{H},-0.6510129356,1.1136913083,0.1988614913 \backslash \mathrm{C},-2.338242845$ $3,2.0691803658,-0.5575451467 \backslash 0,-2.1024648249,0.8607981202,-0.792030069$ $6 \backslash 0,-3.437492463,2.6793138565,-1.0543127703 \backslash \mathrm{C},-4.2812542854,1.85670635$ $81,-1.8441803348 \backslash \mathrm{H},-5.1045508132,2.4919060189,-2.1578691789 \backslash \mathrm{H},-4.65568$ $97444,1.0132675917,-1.2661070794 \backslash \mathrm{H},-3.7492385777,1.4735040354,-2.71362$ $64977 \backslash \mathrm{H},-0.3085673546,1.8812379889,1.6679041047 \backslash \backslash V e r s i o n=E S 64 L-G 16 \operatorname{RevB}$ $.01 \backslash$ State $=1-A \backslash H F=-323.6591761 \backslash R M S D=3.958 \mathrm{e}-09 \backslash \mathrm{RMSF}=9.248 \mathrm{e}-06 \backslash$ Dipole $=1.5$ $46031,0.1801754,1.0274208 \backslash$ Quadrupole $=6.8823021,-7.2093464,0.3270443,0$. 6881173,6.9362246,-0.6393578\PG=C01 [X(C3H7N1O2)]\\@

\section{CA1.xyz-M062X-MG3S}

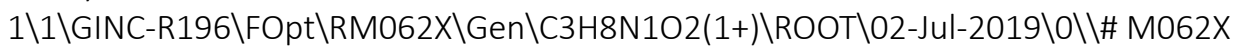

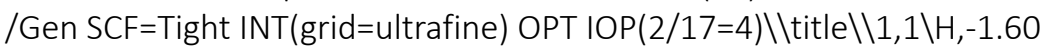
$88763082,3.7021208302,0.4884477093 \backslash C,-1.7398994072,2.6345690223,0.6409$ $475185 \backslash \mathrm{N},-0.406263008,1.9674875015,0.7928710107 \backslash \mathrm{H},-0.3569233015,1.2787$ $878222,0.0133056814 \backslash \mathrm{H},-0.3095785836,1.4710098676,1.6785951831 \backslash \mathrm{H},-2.342$ $3439431,2.4732645208,1.5303985029 \backslash C,-2.3884888063,1.9864041656,-0.5802$ $086261 \backslash 0,-1.8084791917,1.1247767411,-1.1892116522 \backslash 0,-3.5629509114,2.48$ 
$19824706,-0.8214497424 \backslash \mathrm{C},-4.273250228,1.9382418588,-1.96292509 \backslash \mathrm{H},-5.21$ $39089427,2.4727941648,-1.9929561572 \backslash H,-4.427804024,0.8735362911,-1.813$ $6039899 \backslash H,-3.6902711057,2.1124589079,-2.8626837332 \backslash H, 0.3747377614,2.61$ $81058357,0.7089133852 \backslash \mid$ Version=ES64L-G16RevB.01\State=1-A\HF=-324.0692 $337 \backslash \mathrm{RMSD}=4.165 \mathrm{e}-09 \backslash \mathrm{RMSF}=1.006 \mathrm{e}-05 \backslash \mathrm{Dipole}=1.8904287,0.4710944,1.8870029$ lQuadrupole=7.3591489,-8.1139042,0.7547554,1.5407402,11.6375134,-0.714 6979\PG=C01 [X(C3H8N1O2)]入\@

\section{CA2-BASE.xyz-M062X-MG3S}

$1 \backslash 1 \backslash G I N C-R 203 \backslash F O p t \backslash R M 062 X \backslash G e n \backslash C 6 H 11 N 1 O 2 \backslash R O O T \backslash 02-J u l-2019 \backslash 0 \backslash \backslash \#$ M062X/Ge n SCF=Tight INT(grid=ultrafine) OPT IOP(2/17=4)\title $\backslash 0,1 \backslash H, 3.125177$ $7711,-2.9666872745,0.8001867796 \backslash C, 3.6352034722,-2.3755035745,0.0647813$ $598 \backslash \mathrm{N}, 4.9709108068,-2.5212737245,-0.1586543968 \backslash \mathrm{H}, 5.278737916,-1.876109$ $2683,-0.8908107051 \backslash C, 3.0285377315,-1.3904399601,-0.7560788486 \backslash 0,3.6255$ $435073,-0.7220470028,-1.5926220645 \backslash 0,1.7039698005,-1.2476188354,-0.532$ $3353809 \backslash \mathrm{C}, 1.0605963413,-0.2696000713,-1.3359778289 \backslash \mathrm{H}, 0.0168508785,-0.2$ $782154234,-1.0374440126 \backslash \mathrm{H}, 1.1547087756,-0.5156696023,-2.3924234313 \backslash \mathrm{H}, 1$ $.4948789976,0.714541745,-1.167655169 \backslash C, 5.8477613073,-3.3087817737,0.38$ $81959696 \backslash C, 7.2722857087,-3.2648451075,-0.0480241669 \backslash C, 5.3796964893,-4$. $2381524533,1.448978246 \backslash \mathrm{H}, 7.9275569907,-3.0150558618,0.7895894937 \backslash \mathrm{H}, 7.4$ $270595328,-2.5269820173,-0.8339083175 \backslash \mathrm{H}, 7.5933249103,-4.2385976141,-0$. $4248588919 \backslash \mathrm{H}, 4.9370545789,-3.6811362538,2.2812863508 \backslash \mathrm{H}, 6.197559689,-4$. 84504964,1.8278071149\Н,4.5968347945,-4.8994762862,1.0634178995\\Versi on=ES64L-G16RevB.01 \State=1-A \HF=-440.3958015 \RMSD=1.215e-09 \RMSF=1.29 9e-05\Dipole=1.0377665,-0.9564504,0.6736869 \Quadrupole $=4.759009,-1.536$ 1665,-3.2228426,-4.168945,1.9426639,-0.661312\PG=C01 [X(C6H11N1O2)]\\@

\section{CA2.xyz-M062X-MG3S}

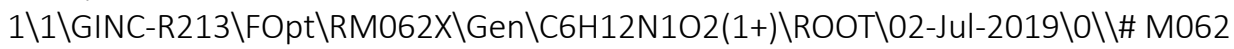

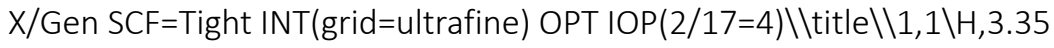
$71481629,-2.333196016,1.1571556283 \backslash C, 3.5495943967,-2.520173508,0.10097$ $28686 \backslash N, 4.981548303,-2.5563804453,-0.1678763242 \backslash H, 5.2628511984,-1.8632$ $53879,-0.8679817464 \backslash \mathrm{H}, 3.0787245282,-3.4631029598,-0.1780013866 \backslash \mathrm{C}, 2.977$ $0783891,-1.390205651,-0.7454035687 \backslash 0,3.6710301197,-0.7387234682,-1.477$ 8106866\0,1.6911586254,-1.2623286389,-0.5547139689\C,1.0354706914,-0.2 $213258236,-1.3128499292 \backslash \mathrm{H},-0.0071766438,-0.2701111918,-1.025354977 \backslash \mathrm{H}, 1$ $.1598956596,-0.4143371855,-2.374734584 \backslash H, 1.4694011129,0.7409936524,-1$. $0559783778 \backslash \mathrm{C}, 5.854104654,-3.3227429191,0.3809719552 \backslash \mathrm{C}, 7.2791539823,-3$. $2118711271,-0.0088138024 \backslash \mathrm{C}, 5.4426176733,-4.3039586312,1.4180225288 \backslash \mathrm{H}, 7$ $.8775545951,-3.046693802,0.8892013315 \backslash H, 7.4616942358,-2.417752766,-0.7$ $27611093 \backslash H, 7.600526191,-4.1688703563,-0.4254079971 \backslash H, 5.2280676979,-3.7$ $64015652,2.3453264313 \backslash \mathrm{H}, 6.2384543248,-5.0154960448,1.6140836555 \backslash H, 4.53$ $55521024,-4.8341535867,1.1298340427 \backslash \backslash V e r s i o n=E S 64 L-G 16 R e v B .01 \backslash S t a t e=1-$ $A \backslash H F=-440.7979387 \backslash R M S D=3.957 e-09 \backslash R M S F=7.855 e-07 \backslash D i p o l e=1.2971097,-1.39$ 99546,1.0007961 \Quadrupole=10.3466751,-2.8388524,-7.5078228,-11.543688 ,5.9456415,-4.3670921\PG=C01 [X(C6H12N1O2)]\\@

\section{CA3-BASE.xyz-M062X-MG3S}

$1 \backslash 1 \backslash G I N C-R 427 \backslash F O p t \backslash R M 062 X \backslash G e n \backslash C 9 H 10 N 2 \backslash R O O T \backslash 02-J u l-2019 \backslash 0 \backslash \backslash \#$ M062X/Gen SCF=Tight INT(grid=ultrafine) OPT IOP(2/17=4)\|title \0,1\C,0.29915691 $13,3.7813109921,-0.3590046445 \backslash C,-0.0469482412,2.4278634797,-0.32338895$ $7 \backslash C, 0.7234151712,1.502004588,0.3671519878 \backslash C, 1.4279261175,4.2568804899$, $0.2946320783 \backslash C, 2.1956555989,3.3305413713,0.9834209526 \backslash C, 1.8499803838,1$ $.9792040635,1.0191360152 \backslash N, 3.3573645449,3.4364357775,1.7304685818 \backslash \mathrm{C}, 3$. 
$7675889556,2.2412039826,2.2366387071 \backslash \mathrm{N}, 2.8273034708,1.3661420101,1.785$ $8484293 \backslash C, 4.0546104039,4.6831166606,1.9480438651 \backslash H, 3.4104169502,5.3958$ $889298,2.4638355219 \backslash H, 4.3707956008,5.1143433859,0.9977243286 \backslash H, 4.92577$ $52731,4.4716439671,2.5593945179 \backslash C, 2.8441191719,-0.0498685359,2.0727859$ $407 \backslash H, 1.9379582602,-0.3421046389,2.6042885354 \backslash \mathrm{H}, 3.7109395898,-0.250431$ $4422,2.6939084558 \backslash \mathrm{H}, 2.9148600953,-0.6258453447,1.1495660249 \backslash \mathrm{H},-0.32603$ $98782,4.4717672094,-0.9075645588 \backslash \mathrm{H},-0.9336242247,2.0958717814,-0.84503$ 10349\H,0.4549502431,0.4547750512,0.3941390319\Н,1.6951256019,5.304406 $2212,0.2665562208 \backslash \backslash$ Version=ES64L-G16RevB.01 \State $=1-A \backslash H F=-458.4033237 \backslash$ RMSD $=5.590 \mathrm{e}-09 \backslash \mathrm{RMSF}=2.205 \mathrm{e}-05 \backslash$ Dipole $=-0.5618115,0.1331874,-0.3983433 \backslash \mathrm{Q}$ uadrupole $=-3.6499426,7.8954532,-4.2455107,3.1525851,-0.343281,-0.25488$ $31 \backslash P G=C 01[X(C 9 H 10 N 2)] \backslash \backslash @$

\section{CA3.XYz-M062X-MG3S}

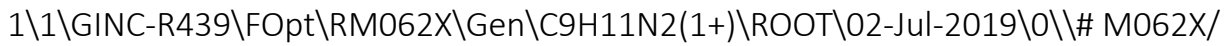
Gen SCF=Tight INT(grid=ultrafine) OPT IOP(2/17=4)\\title \} \backslash 1 , 1 \backslash C , 0 . 3 0 0 0 $170975,3.7863143585,-0.3450839678 \backslash C,-0.0485541868,2.425158622,-0.31023$ $75371 \backslash C, 0.7147827377,1.493816446,0.3640567254 \backslash C, 1.4251410866,4.2676524$ 48,0.2930094156\C,2.1935051883,3.3290802714,0.9719222529\C,1.847827792 $4,1.9792984894,1.0065045042 \backslash \mathrm{N}, 3.3652304319,3.455860807,1.7082562895 \backslash \mathrm{C}$, $3.7017546676,2.2535879485,2.1573692325 \backslash \mathrm{N}, 2.8235754147,1.3407759843,1.7$ $624187293 \backslash \mathrm{C}, 4.086237323,4.7025907948,1.9368986926 \backslash \mathrm{H}, 4.5705580803,2.046$ $5570339,2.761078197 \backslash \mathrm{H}, 3.4366876307,5.4031488352,2.4562456159 \backslash \mathrm{H}, 4.39404$ $09142,5.119994339,0.9811533728 \backslash \mathrm{H}, 4.962243552,4.4982909562,2.5446641836$ \C,2.8596708771,-0.0863677997,2.0597792635\Н,1.9595959939,-0.362808781 $2,2.6037107336 \backslash \mathrm{H}, 3.7331930899,-0.2989534789,2.6682784106 \backslash \mathrm{H}, 2.917850488$ $7,-0.6458567226,1.1291837508 \backslash \mathrm{H},-0.3326172283,4.4737633477,-0.887708172$ $1 \backslash \mathrm{H},-0.9402600891,2.1009513676,-0.8269608107 \backslash \mathrm{H}, 0.4449525584,0.44774635$ $01,0.3895951417 \backslash \mathrm{H}, 1.6912965794,5.3145983828,0.2649359763 \backslash \backslash V e r s i o n=E S 64$ L-G16RevB.01\State=1-A \HF=-458.8243799 \RMSD $=4.731 \mathrm{e}-09 \backslash \mathrm{RMSF}=6.118 \mathrm{e}-06 \backslash \mathrm{D}$ ipole $=1.2331559,-0.2938594,0.8568728 \backslash$ Quadrupole $=1.1941058,6.34628,-7.5$ 403858,1.7030495,9.8587583,-2.894253\PG=C01 [X(C9H11N2)]\\@

\section{CA4-BASE.xyz-M062X-MG3S}

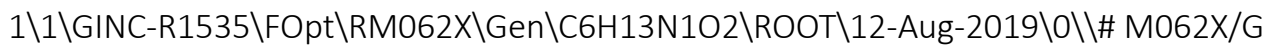

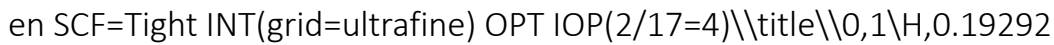
$77042,-1.875604495,-0.0002170275 \backslash C, 0.145855248,-0.8042000969,0.0014768$ $78 \backslash \mathrm{N}, 1.4377530007,-0.0906840896,-0.000144554 \backslash \mathrm{C}, 1.5880937494,0.77435444$ $9,1.2146563596 \backslash C, 1.5874730095,0.7709560041,-1.2174656111 \backslash C,-1.01235199$ $33,-0.0435111459,0.0002453157 \backslash 0,-1.0916999927,1.1941063821,-0.00052015$ $81 \backslash 0,-2.1479632071,-0.8102541467,0.0002647697 \backslash C,-3.3597296332,-0.07993$ $71603,-0.0000066056 \backslash \mathrm{H},-4.1546241989,-0.82101816,0.0005771439 \backslash \mathrm{H},-3.4388$ $722768,0.5524816424,-0.8838312043 \backslash \mathrm{H},-3.4385869142,0.5535993326,0.88303$ 4684\C,2.5249692578,-1.0977892282,0.0010348825\H,0.7556135514,1.471252 $0982,1.2164036124 \backslash \mathrm{H}, 2.5431220194,1.296968005,1.1684350552 \backslash \mathrm{H}, 1.54206905$ $63,0.1266919192,2.0845339908 \backslash H, 0.7550865338,1.4679700442,-1.220617018 \backslash$ $\mathrm{H}, 1.5408328718,0.120880946,-2.0855156326 \backslash \mathrm{H}, 2.5425922704,1.293588537,-1$ $.1733772505 \backslash \mathrm{H}, 2.4209198051,-1.7123245273,0.8910551869 \backslash \mathrm{H}, 3.4850674022,-$ $0.5868122271,0.0002582045 \backslash H, 2.420812736,-1.7146150828,-0.8873930214 \backslash \backslash V$ ersion=ES64L-G16RevB.01 \State $=1-A \backslash H F=-441.561602 \backslash R M S D=3.612 \mathrm{e}-09 \backslash \mathrm{RMSF}=2$ $.538 \mathrm{e}-05 \backslash$ Dipole $=2.342111,-0.2409366,-0.0021753 \backslash$ Quadrupole $=5.7686665,-4$ $.6383044,-1.130362,-0.0616765,-0.0006843,0.0078075 \backslash P G=C 01[X(C 6 H 13 N 1 O 2$ )]\@ 
CA4.xyz-M062X-MG3S

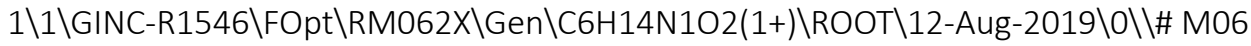
2X/Gen SCF=Tight INT(grid=ultrafine) OPT IOP $(2 / 17=4) \backslash$ title $\backslash 1,1 \backslash H, 0.1$ $600749722,-1.4762055025,0.8808300729 \backslash \mathrm{C}, 0.1722164382,-0.835563288,-0.00$ $02074457 \backslash \mathrm{N}, 1.4601907466,-0.0716697009,0.0000256076 \backslash \mathrm{C}, 1.5844416958,0.78$ $22745541,1.2281403923 \backslash \mathrm{C}, 1.5844451232,0.7830379576,-1.2275585792 \backslash \mathrm{H}, 0.15$ $99802711,-1.4755097623,-0.8817496076 \backslash \mathrm{C},-1.0847962692,0.0267345728,0.00$ $02129139 \backslash 0,-1.1006320753,1.2252170461,0.0006359667 \backslash 0,-2.131957249,-0.7$ $64677982,-0.0002162838 \backslash \mathrm{C},-3.4213132439,-0.1147295101,-0.0000600839 \backslash \mathrm{H},-$ 4.1480145878,-0.9173856366,-0.0005787587\H,-3.5172331761,0.501949094,$0.8894402651 \backslash \mathrm{H},-3.5174793199,0.501017043,0.889939917 \backslash \mathrm{C}, 2.5744270388,-1$ $.0724313501,-0.0002853772 \backslash H, 0.8076547366,1.53757453,1.2097016039 \backslash H, 2.5$ $687074921,1.2421472444,1.2153586627 \backslash \mathrm{H}, 1.4844224289,0.142734393,2.10167$ $21677 \backslash \mathrm{H}, 0.807665608,1.5383331629,-1.208649181 \backslash \mathrm{H}, 1.4844184409,0.1440412$ $477,-2.1014872365 \backslash \mathrm{H}, 2.5687156038,1.2428924923,-1.2144932129 \backslash \mathrm{H}, 2.494668$ $5771,-1.6883236424,0.8914597426 \backslash \mathrm{H}, 3.5180397191,-0.5344580332,-0.000113$ $242 \backslash H, 2.4946740287,-1.6877629299,-0.8924177737 \backslash \backslash V e r s i o n=E S 64 L-G 16 R e v B$.

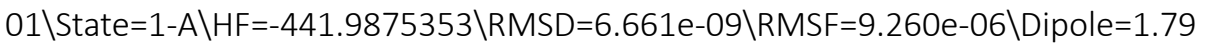
$07484,-0.6750839,-0.0003455 \backslash$ Quadrupole $=12.5181904,-7.8265808,-4.691609$ $6,0.2640264,0.0002626,-0.0013735 \backslash P G=C 01[X(C 6 H 14 N 1 O 2)] \backslash \backslash @$

CA5-BASE.xyz-M062X-MG3S

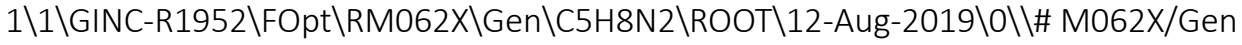

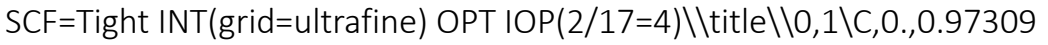
$7231,0.0000854677 \backslash \mathrm{N},-1.0548995723,0.1181956939,0.0000303419 \backslash \mathrm{N}, 1.054899$ $5723,0.1181956939,0.0000303419 \backslash C, 0.6746298162,-1.210091932,0.000043253$ $8 \backslash C,-0.6746298162,-1.210091932,0.0000432538 \backslash C, 2.4312218899,0.576747124$ $5,-0.0000031608 \backslash \mathrm{H}, 1.3786606233,-2.0226567645,0.0000331607 \backslash \mathrm{H}, 2.62224764$ $8,1.1792065688,0.8846306764 \backslash \mathrm{H}, 2.6222017715,1.1792295482,-0.884631655 \backslash \mathrm{H}$ , 3.0963601249,-0.2834963544,-0.0000313529\C,-2.4312218899,0.5767471245 $,-0.0000031608 \backslash \mathrm{H},-1.3786606234,-2.0226567645,0.0000331607 \backslash \mathrm{H},-3.0963601$ 249,-0.2834963544,-0.0000313529\Н,-2.6222017714,1.1792295482,-0.884631 655 \H,-2.622247648,1.1792065688,0.8846306764\\Version=ES64L-G16RevB.01

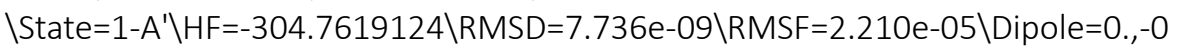
$.9928203,-0.0000635 \backslash Q u a d r u p o l e=6.7862211,-3.6018625,-3.1843587,0 ., 0 .,-$ $0.0001947 \backslash \mathrm{PG}=\mathrm{CS}[\mathrm{SG}(\mathrm{C} 1), \mathrm{X}(\mathrm{C} 4 \mathrm{H} 8 \mathrm{~N} 2)] \backslash \backslash @$

\section{CA5.XYz-M062X-MG3S}

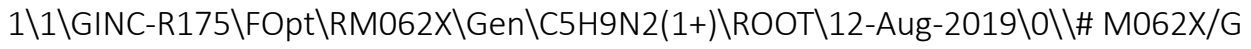

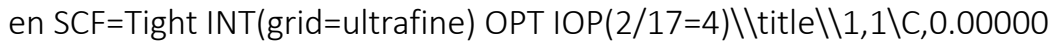

$72203,-0.8581801269,0.0000112542 \backslash \mathrm{N},-1.0803327936,-0.0856848065,0.00001$ $68973 \backslash \mathrm{N}, 1.0803372129,-0.0856707919,0.000005611 \backslash \mathrm{C}, 0.6775534589,1.228822$ $7067,-0.0000090866 \backslash C,-0.677566092,1.228813917,0.0000315949 \backslash C, 2.4685205$ $761,-0.5499925692,0.0000318027 \backslash \mathrm{H}, 1.3828341891,2.0405019306,-0.00002575$ $85 \backslash \mathrm{H}, 2.4741863043,-1.6353013292,-0.0002941575 \backslash \mathrm{H}, 2.968759408,-0.1816563$ $92,0.8912213987 \backslash \mathrm{H}, 2.9689464432,-0.1811302578,-0.8908337742 \backslash \mathrm{H}, 0.0000142$ $043,-1.9349100079,0.0000112542 \backslash C,-2.4685101333,-0.5500245921,-0.000009$ $2944 \backslash \mathrm{H},-1.3828573516,2.0404839916,0.0000482669 \backslash \mathrm{H},-2.9687537434,-0.1816$ $949042,-0.8911988903 \backslash \mathrm{H},-2.9689407854,-0.1811687724,0.8908562826 \backslash \mathrm{H},-2.4$ 741617823,-1.6353334254,0.0003166658||Version=ES64L-G16RevB.01\State=1 $-A \backslash H F=-305.1872533 \backslash R M S D=9.635 e-09 \backslash R M S F=3.793 e-05 \backslash D i p o l e=0.0000021,-0.3$ $226704,0.1$ Quadrupole $=8.9465771,-0.0610651,-8.885512,0.0000584,-0.00002$ $33,0 . \mid P G=C 02[\mathrm{C} 2(\mathrm{C} 1 \mathrm{H} 1), \mathrm{X}(\mathrm{C} 4 \mathrm{H} 8 \mathrm{~N} 2)] \backslash \backslash @$ 
CAR1-BASE.xyz-M062X-MG3S

$1 \backslash 1 \backslash G I N C-R 197 \backslash F O p t \backslash R M 062 X \backslash G e n \backslash C 8 H 8 N 1 O 2(1-) \backslash R O O T \backslash 02-J u l-2019 \backslash 0 \backslash \backslash \#$ M062X

/Gen SCF=Tight INT(grid=ultrafine) OPT IOP(2/17=4)\title \-1,1\H,1.49

$05281587,0.638014074,1.0923477038 \backslash \mathrm{C}, 0.8657745806,0.8514774832,1.949674$

$5169 \backslash \mathrm{C}, 1.4104007745,0.9785843889,3.221271675 \backslash \mathrm{H}, 2.4782903629,0.86383146$

$53,3.368336237 \backslash \mathrm{C}, 0.6087847655,1.2516408995,4.3158078032 \backslash H, 1.0843748332$ ,1.3401131263,5.2810814906\C,-0.7975881304,1.4168048287,4.2125315925\C $,-1.5353992679,1.6986907217,5.4172675047 \backslash C,-1.3259609519,1.2815951218$, $2.9022568804 \backslash \mathrm{H},-2.3854344477,1.3962494009,2.7615135123 \backslash \mathrm{C},-0.510110685$, $1.0082358695,1.8176783951 \backslash \mathrm{H},-0.9677986836,0.9153920905,0.839252257 \backslash \mathrm{N}$,2.8861109725,1.8774008595,5.4471128533\0,-3.4671101763,2.1215212356,6. 5390917056\0,-3.5731275672,1.8032582529,4.3974898624\C,-0.8108461119,1 $.8081390047,6.724989746 \backslash \mathrm{H},-1.5229909182,2.0221723598,7.5128715611 \backslash \mathrm{H},-0$ $.0599507947,2.6061029312,6.7086530099 \backslash \mathrm{H},-0.2822947682,0.8816358859,6.9$ 761716933\|Version=ES64L-G16RevB.01 \State $=1-A \backslash H F=-514.781128 \backslash$ RMSD $=8.87$ 0e-09\RMSF $=4.207 \mathrm{e}-06 \backslash$ Dipole $=2.469271,-0.452252,-0.8643081 \backslash$ Quadrupole $=-$ $8.4333503,7.3758704,1.0574799,3.6910783,9.9579496,-2.3938423 \backslash P G=C 01[X$ (C8H8N1O2)]\\@

CAR1.xyz-M062X-MG3S

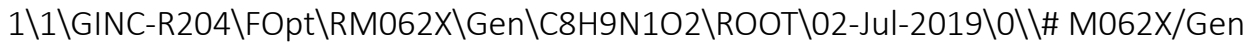

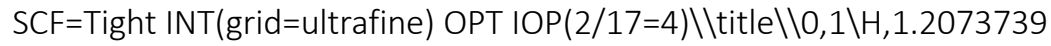
$957,0.4164859755,1.0967981445 \backslash C, 0.7308285346,0.7658315302,2.0023987389$ IC,1.3031972418,1.7932304638,2.7369091772\H,2.2261587983,2.2494824539, 2.4067133994\С,0.6864174903,2.2434926703,3.8961412398\Н,1.1260039457,3 $.053386866,4.4642979278 \backslash C,-0.4975836022,1.6620746054,4.3325100999 \backslash C,-1$ $.1424932717,2.1474417926,5.6089545094 \backslash C,-1.0709194033,0.6323337597,3.5$ $910155609 \backslash \mathrm{H},-2.0047886641,0.1911266759,3.9144727711 \backslash \mathrm{C},-0.4590225554,0$. $1895037551,2.42903011 \backslash \mathrm{H},-0.9122674924,-0.6061651619,1.8540478993 \backslash \mathrm{N},-2$. $4708631515,2.7882406436,5.2366057114 \backslash 0,-3.4340810886,2.063816766,5.139$ $5652588 \backslash 0,-2.4634129818,3.9782201985,5.0328297394 \backslash C,-1.3697246026,1.06$ $14733489,6.6425074002 \backslash H,-1.8667723917,1.4613893127,7.5247146581 \backslash \mathrm{H},-0.4$ $020410705,0.6566662941,6.9335847267 \backslash \mathrm{H},-1.9776875636,0.2590570115,6.233$ $6568096 \backslash \mathrm{H},-0.5688021669,2.9756510383,6.0147961175 \backslash \backslash$ Version=ES64L-G16Re

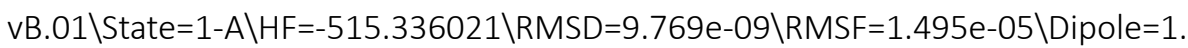
$3296175,-0.8155114,0.1951294 \backslash$ Quadrupole $=-3.4943524,-2.3347144,5.829066$ 8,4.8295437,-0.7357182,1.733998\PG=C01 [X(C8H9N1O2)]\\@

\section{CAR2-BASE.xyz-M062X-MG3S}

$1 \backslash 1 \backslash G I N C-R 568 \backslash F O p t \backslash R M 062 X \backslash G e n \backslash C 7 H 5 N 2 O 4(1-) \backslash R O O T \backslash 02-J u l-2019 \backslash 0 \backslash \backslash \#$ M062X /Gen SCF=Tight INT(grid=ultrafine) OPT IOP(2/17=4)\title \-1,1\N,1.41 $56706071,2.3892268368,-0.9598511648 \backslash C, 0.5487204992,1.306832162,-0.6115$ $631496 \backslash \mathrm{C},-0.6575527412,1.5568934813,0.0447484388 \backslash \mathrm{H},-0.9180373866,2.578$ $1762407,0.2810049865 \backslash \mathrm{C},-1.4991455931,0.526474281,0.3847448655 \backslash \mathrm{H},-2.426$ $8083434,0.7292153515,0.8906612332 \backslash C,-1.167740789,-0.8262422448,0.07841$ 75174\C,-1.9298056829,-1.9675568032,0.3662094876\H,-1.5810496033,-2.94 $65494547,0.0875548851 \backslash \mathrm{C}, 0.076459706,-1.0349495479,-0.5931476928 \backslash \mathrm{H}, 0.36$ $05004742,-2.049850095,-0.8409601934 \backslash C, 0.9113675512,-0.0063079797,-0.92$ $97974373 \backslash \mathrm{H}, 1.847228095,-0.1861494035,-1.4374610554 \backslash 0,1.0786565395,3.53$ $1086487,-0.6712549601 \backslash 0,2.4681487626,2.1365778613,-1.5360876796 \backslash \mathrm{N},-3.1$ 480189348,-2.0052002221,1.0007145945\0,-3.699500376,-0.9625014389,1.39 $17173155 \backslash 0,-3.6807227846,-3.1176155118,1.171350009$ \Version=ES64L-G16R evB.01\State $=1-A \backslash H F=-680.0072246 \backslash R M S D=5.372 e-09 \backslash R M S F=6.268 e-05 \backslash D i p o l e=$ $1.3996814,0.255483,-0.708456 \backslash$ Quadrupole $=-10.452603,-4.3184091,14.77101$ 


\section{1,-26.7583189,13.7894075,11.5534794\PG=C01 [X(C7H5N2O4)]\\@}

CAR2.xyz-M062X-MG3S

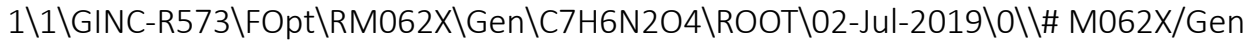
SCF=Tight INT(grid=ultrafine) OPT IOP(2/17=4)\\title \} \backslash 0 , 1 \backslash N , 1 . 5 4 3 7 4 4 8 $748,2.2550669838,-0.6938680657 \backslash C, 0.4927467792,1.2257516424,-0.54770640$ $24 \backslash \mathrm{C},-0.801994588,1.6341923473,-0.2872388788 \backslash \mathrm{H},-1.0234223515,2.6859539$ 474,-0.1910041726\C,-1.7820503077,0.664367866,-0.1577544555\H,-2.80292 $68499,0.9535309166,0.0531416165 \backslash C,-1.4585024165,-0.6819534797,-0.29189$ 0887\C,-2.5215940774,-1.7294386445,-0.119396545\H,-3.5136973984,-1.346 $9048915,-0.3339931506 \backslash \mathrm{H},-2.306107528,-2.6293595485,-0.6859422928 \backslash \mathrm{C},-0$. $1445817172,-1.0626195817,-0.5470116609 \backslash H, 0.1028416458,-2.1119598095,-0$ $.6364291557 \backslash \mathrm{C}, 0.8462482544,-0.1046370784,-0.678676652 \backslash \mathrm{H}, 1.8730219989,-$ $0.3692902862,-0.8791316113 \backslash 0,1.210154778,3.4110315755,-0.5727660694 \backslash 0$, $2.6673392648,1.8730325371,-0.92568517 \backslash \mathrm{N},-2.5409237222,-2.147911331,1.3$ $30074494 \backslash 0,-3.2750805625,-1.5347325159,2.065980772 \backslash 0,-1.7915960766,-3$. 0389906493,1.6491882871 \|Version=ES64L-G16RevB.01 \State=1-A \HF=-680.52 73031 \RMSD=6.314e-09\RMSF=5.182e-06\Dipole $=-1.0729078,-0.6953192,-1.15$ 15505\Quadrupole=1.5819,-1.5489582,-0.0329418,-7.4730068,6.5619404,8.0 $766271 \backslash P G=C 01[X(C 7 H 6 N 204)] \backslash \backslash @$

CAR3-BASE.xyz-M062X-MG3S

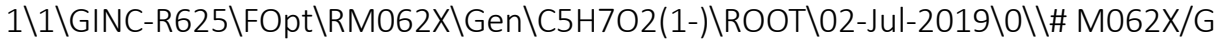

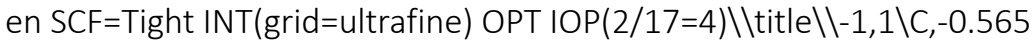
$3961726,-0.7315963638,-0.0984799916 \backslash \mathrm{H},-0.276378984,-0.9517146729,-1.11$ 79002657\C,-1.2991605528,-1.7607871732,0.557454099\C, $-0.1933016774,0.5$ $120437653,0.4468397009 \backslash 0,-1.5943300379,-2.8368178064,0.0155657239 \backslash 0,-0$ $.4254542042,0.9547120196,1.5888988957 \backslash C,-1.7471201656,-1.5372654931,1$. $994831335 \backslash \mathrm{H},-0.8884450404,-1.3396122855,2.6361064084 \backslash \mathrm{H},-2.2884826854,-$ $2.4216965112,2.327246395 \backslash \mathrm{H},-2.3710508962,-0.6465767605,2.0661033747 \backslash \mathrm{C}$, $0.5993058539,1.4447370458,-0.478671707 \backslash \mathrm{H}, 1.5531878308,1.6782215226,-0$. $0026772416 \backslash \mathrm{H}, 0.0499765968,2.3822045145,-0.5804985797 \backslash \mathrm{H}, 0.781000135,1.0$ 221581989,-1.4660881469 \|Version=ES64L-G16RevB.01 \State=1-A \HF=-345.21 $35508 \backslash \mathrm{RMSD}=7.018 \mathrm{e}-09 \backslash \mathrm{RMSF}=1.512 \mathrm{e}-05 \backslash \mathrm{Dipole}=0.4049969,0.7590673,-0.1288$ 998\Quadrupole $=5.1884513,-8.2926724,3.1042211,-5.0849899,-1.0504446,-5$ .5820972\PG=C01 [X(C5H7O2)]\\@

CAR3.xyz-M062X-MG3S

$1 \backslash 1 \backslash G I N C-R 644 \backslash F O p t \backslash R M 062 X \backslash G e n \backslash C 5 H 8 O 2 \backslash R O O T \backslash 02-J u l-2019 \backslash 0 \backslash \backslash \#$ M062X/Gen S CF=Tight INT(grid=ultrafine) OPT IOP(2/17=4)\title \\0,1\C,-0.56816335

$81,-0.7117809015,-0.1470898733 \backslash \mathrm{H},-0.7428674497,-0.7196036631,-1.222565$ $4079 \backslash \mathrm{H}, 0.3671244259,-1.2463018509,0.0460782835 \backslash \mathrm{C},-1.7328115801,-1.3944$ $065954,0.5520100153 \backslash C,-0.3602956596,0.723275574,0.3239141926 \backslash 0,-2.7769$ 650156,-1.5449407271,-0.0255772662\0,-0.8307413245,1.1349132551,1.3512 $712717 \backslash \mathrm{C},-1.5055713168,-1.8562390796,1.9645846781 \backslash \mathrm{H},-0.7086618819,-2.6$ $023475603,1.9802203226 \backslash \mathrm{H},-2.4191445127,-2.2833351047,2.3666166858 \backslash \mathrm{H},-1$ $.1805545004,-1.009911522,2.5694556104 \backslash C, 0.4725906211,1.5899722898,-0.5$ $849927322 \backslash \mathrm{H}, 0.7067479606,2.5282357322,-0.091483758 \backslash \mathrm{H},-0.092036358,1.78$ 72807005,-1.4979129735\H,1.38665995,1.072349453,-0.877079049\\Version= ES64L-G16RevB.01 \State=1-A \HF=-345.7622517\RMSD=7.083e-09 \RMSF=1.006e05\Dipole $=1.4840954,-0.127123,-0.3828234 \backslash$ Quadrupole $=-1.6828649,0.43860$ 46,1.2442603,-1.3558097,-2.6773271,-4.5255369\PG=C01 [X(C5H8O2)]\\@ 


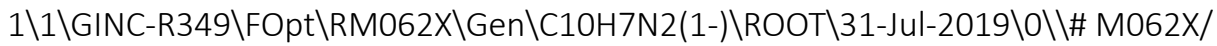
Gen SCF=Tight INT(grid=ultrafine) OPT IOP(2/17=4)\|title \-1,1\C,0.903 $3353745,0.0423405845,-2.9045065504 \backslash C, 0.1043071837,0.2517523322,-1.6454$ $44232 \backslash \mathrm{C},-1.2841343255,0.3440612899,-1.6614684868 \backslash \mathrm{H},-1.8080927471,0.262$ 9032817,-2.6082919458\C,-2.0202677797,0.5367634074,-0.5036754134\Н,-3. $0996268115,0.6033318866,-0.5578087548 \backslash \mathrm{C},-1.3944751674,0.6489455809,0.7$ $507800422 \backslash C,-2.150621551,0.8500154279,1.965303588 \backslash C,-3.5508542253,0.94$ $01605084,1.9276406556 \backslash C,-1.4988844578,0.9586805443,3.2036774234 \backslash C, 0.01$ $18856599,0.5548441044,0.762137314 \backslash \mathrm{H}, 0.532937502,0.6360858693,1.7078755$ $961 \backslash \mathrm{C}, 0.7294576685,0.362426737,-0.4032369454 \backslash \mathrm{H}, 1.8116102343,0.29574661$ $97,-0.3480782416 \backslash \mathrm{N},-4.7050018229,1.007180903,1.8481753786 \backslash \mathrm{N},-0.9188718$ $097,1.0450399377,4.2032155406 \backslash H, 0.2488234712,-0.0210940615,-3.77399389$ $03 \backslash \mathrm{H}, 1.6054574115,0.8613561549,-3.0775488734 \backslash \mathrm{H}, 1.4882961924,-0.8794711$ 084,-2.8623322046\|Version=ES64L-G16RevB.01 \State $=1-A \mid H F=-494.7864782 \backslash$ $\mathrm{RMSD}=6.477 \mathrm{e}-09 \backslash \mathrm{RMSF}=4.310 \mathrm{e}-05 \backslash$ Dipole $=2.0376368,-0.5371031,-3.2298781 \backslash \mathrm{Q}$ uadrupole $=-3.5216766,13.0079561,-9.4862795,1.8938655,6.0701268,-3.2637$ $25 \backslash P G=C 01[X(C 10 H 7 N 2)] \backslash \backslash @$

\section{CAR4.XYZ-M062X-MG3S}

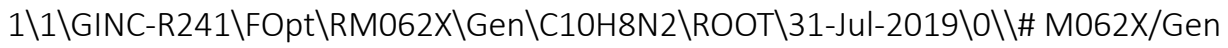

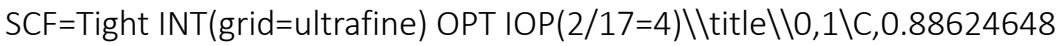
$8,0.155888476,-2.8781204219 \backslash C, 0.0716577749,0.2360853229,-1.6171778701 \backslash$ $\mathrm{C},-1.3069903066,0.3991498905,-1.6547550627 \backslash \mathrm{H},-1.8111369134,0.460622788$ 6,-2.6105577294\C,-2.0542764751,0.4907176601,-0.4870732978\Н,-3.126350 $065,0.6256255993,-0.5448213329 \backslash C,-1.4222087555,0.4175293249,0.74309859$ $18 \backslash C,-2.2208821636,0.4482759475,2.0487713965 \backslash C,-3.5869177131,0.9537795$ $687,1.8688108292 \backslash C,-1.5324198957,1.2196518926,3.0937134338 \backslash C,-0.041822$ $4564,0.2563208169,0.7992607672 \backslash \mathrm{H}, 0.4622790514,0.21368018,1.7567350675 \backslash$ $\mathrm{C}, 0.6914339573,0.165814686,-0.3698272534 \backslash \mathrm{H}, 1.7659531949,0.0432792492,-$ $0.3150518635 \backslash N,-4.6579799582,1.3185888886,1.7010597946 \backslash N,-0.9611355802$ ,1.7890787807,3.9049068293\H,-2.31523303333,-0.5744565462,2.4284584404\ $\mathrm{H}, 0.2485542855,0.0761879949,-3.7558978621 \backslash \mathrm{H}, 1.507747595,1.0449343286$,$2.9917208709 \backslash \mathrm{H}, 1.5514009691,-0.7070548499,-2.8579115857 \backslash \backslash V e r s i o n=E S 64 \mathrm{~L}$

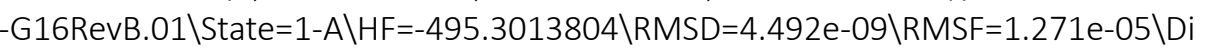
pole $=0.9955026,-1.1899066,-1.3728938 \backslash$ Quadrupole $=-1.7383662,0.6715976,1$ $.0667686,1.5418417,-1.4184857,-5.6659785 \backslash P G=C 01[X(C 10 H 8 N 2)] \backslash \backslash @$

\section{CAR5-BASE.xyz-M062X-MG3S}

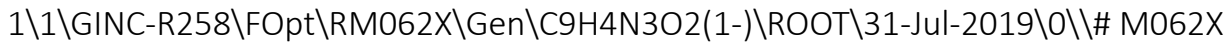
/Gen SCF=Tight INT(grid=ultrafine) OPT IOP(2/17=4)\title \-1,1\N,0.89 $03734821,-2.7559964098,-0.0989477865 \backslash C,-0.0061359973,-1.6419098072,-0$. $2213751083 \backslash \mathrm{C},-1.3851817243,-1.8516217466,-0.174909941 \backslash \mathrm{H},-1.7592324756$, $-2.8563707256,-0.0466065064 \backslash C,-2.2422233669,-0.7873464374,-0.291834657$ $1 \backslash \mathrm{H},-3.3107115184,-0.9519877058,-0.2555072088 \backslash \mathrm{C},-1.7594959517,0.536941$ $1172,-0.4608088015 \backslash \mathrm{C},-2.6468894286,1.6398088481,-0.5819861779 \backslash \mathrm{C},-4.044$ $002518,1.4528514422,-0.5373878746 \backslash C,-2.1539138881,2.9503980177,-0.7498$ 069956\C,-0.3512617258,0.7110993204,-0.5033823241\Н,0.04697062,1.70867 $97781,-0.6312201048 \backslash \mathrm{C}, 0.5050578076,-0.3537622757,-0.3863503185 \backslash \mathrm{H}, 1.575$ $2259715,-0.2140159582,-0.4195963281 \backslash \mathrm{N},-1.7137350116,4.0098325175,-0.88$ $78249245 \backslash N,-5.1826801285,1.2612427576,-0.494100023 \backslash 0,2.0947113586,-2.5$ $454884044,-0.1428024261 \backslash 0,0.4170444949,-3.874914328,0.0447375067 \backslash \backslash V e r s$ ion=ES64L-G16RevB.01 \State=1-A \HF=-660.0070101 \RMSD =8.053e-09\RMSF=4.3 27e-05\Dipole $=1.1090725,-1.3782639,0.1510136 \backslash$ Quadrupole $=-7.082092,-16$. $1942021,23.2762942,21.2870134,-2.1125555,4.5693827 \backslash P G=C 01$ [X(C9H4N3O2) 
]\@

CAR5.XYZ-M062X-MG3S

$1 \backslash 1 \backslash G I N C-R 258 \backslash F O p t \backslash R M 062 X \backslash G e n \backslash C 9 H 5 N 3 O 2 \backslash R O O T \backslash 31-J u l-2019 \backslash 0 \backslash \backslash \#$ M062X/Gen

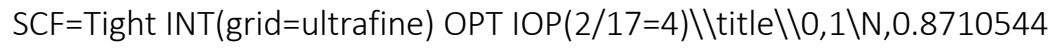
$391,-2.7606959722,-0.2186584593 \backslash C,-0.0549837745,-1.6084468323,-0.22535$ 72052 \C,-1.4133307567,-1.8480659378,-0.146516357\\,-1.7775453663,-2.86 $21402948,-0.0859840755 \backslash \mathrm{C},-2.2767369161,-0.7651447916,-0.1540144316 \backslash \mathrm{H},-$ $3.3442421878,-0.9327451998,-0.1053869525 \backslash C,-1.7708876641,0.5265347238$, $-0.238702576 \backslash C,-2.7254909643,1.722586553,-0.172343624 \backslash C,-4.0616483148$, $1.4000267436,-0.6881418332 \backslash C,-2.187899621,2.9048584998,-0.8569164332 \backslash C$ $,-0.4004433632,0.7417876303,-0.3228137657 \backslash \mathrm{H},-0.0083344353,1.7464950221$ $,-0.4055023373 \backslash C, 0.4714341457,-0.3343121761,-0.3160769944 \backslash H, 1.53978593$ $04,-0.1978566579,-0.3844157758 \backslash \mathrm{H},-2.8539689242,2.000554413,0.879441055$ $5 \backslash \mathrm{N},-1.7311787763,3.823017821,-1.3632283954 \backslash \mathrm{N},-5.1047343337,1.11376097$ $86,-1.059600138 \backslash 0,2.0538335306,-2.521586248,-0.2902365792 \backslash 0,0.38275735$ 24,-3.8637182747,-0.1400851222 \\Version=ES64L-G16RevB.01\State=1-A\HF= $-660.4919317 \backslash \mathrm{RMSD}=5.851 \mathrm{e}-09 \backslash \mathrm{RMSF}=5.354 \mathrm{e}-06 \backslash \mathrm{Dipole}=-0.1745893,0.3346426$ ,1.0469673\Quadrupole=-3.1213529,-5.8770431,8.998396,8.7049746,-4.4956 873,6.1268991\PG=C01 [X(C9H5N3O2)]\\@

CARB1-BASE.xyz-M062X-MG3S

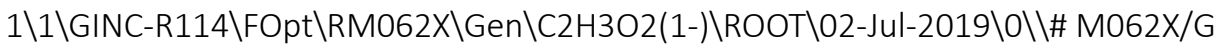
en SCF=Tight INT(grid=ultrafine) OPT IOP(2/17=4)\title \-1,1\H,3.4299 $637135,1.551648215,-0.0637081666 \backslash \mathrm{C}, 2.9900799361,0.886524123,0.68167827$ $68 \backslash \mathrm{H}, 3.5752154526,-0.034932261,0.6768507706 \backslash \mathrm{H}, 3.041568246,1.3505374837$ $, 1.6656860789 \backslash \mathrm{C}, 1.5228189729,0.5635017545,0.277417709 \backslash 0,0.6429085158,0$ $.8701151006,1.1068929093 \backslash 0,1.3952360636,0.0286902263,-0.8433437158 \backslash \backslash V e$ rsion=ES64L-G16RevB.01\State=1-A' $\backslash H F=-228.5127445 \backslash R M S D=6.505 e-09 \backslash R M S F=$ $1.355 \mathrm{e}-05 \backslash$ Dipole $=1.3939918,0.3126543,0.3964187 \backslash$ Quadrupole $=-1.1280983,2$ $.9238686,-1.7957704,-0.0734868,0.8873452,-2.7167591 \backslash P G=C S[S G(C 2 H 1 O 2)$, X(H2)]\\@

\section{CARB1.Xyz-M062X-MG3S}

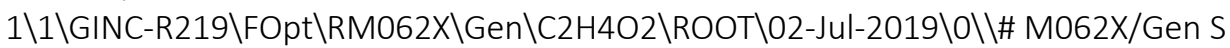

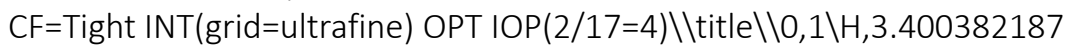

$2,1.5351416028,-0.0930358691 \backslash C, 2.9817953211,0.8795455125,0.6687806605 \backslash$ $\mathrm{H}, 3.5464524747,-0.0510753859,0.6473846677 \backslash \mathrm{H}, 3.0530019929,1.3424781556$, $1.6463180314 \backslash C, 1.5437983355,0.5988922722,0.3512160953 \backslash 0,0.6068486078,0$ $.8436051774,1.0559479221 \backslash 0,1.4062074048,0.0216947015,-0.8588784286 \backslash \mathrm{H}, 0$ $.462933676,-0.1297220361,-0.9995630794 \backslash \backslash V e r s i o n=E S 64 L-G 16 R e v B .01 \backslash S t a t e$ $=1-\mathrm{A} \backslash \mathrm{HF}=-229.0778894 \backslash \mathrm{RMSD}=6.231 \mathrm{e}-09 \backslash \mathrm{RMSF}=7.406 \mathrm{e}-05 \backslash \mathrm{Dipole}=0.539748,-0$. $1438256,-0.4130608 \backslash$ Quadrupole $=0.885584,0.2528357,-1.1384197,1.6563413$, $3.4224455,-0.3430303 \backslash P G=C 01[X(C 2 H 4 O 2)] \backslash \backslash @$

CARB2-BASE.xyz-M062X-MG3S

$1 \backslash 1 \backslash G I N C-R 244 \backslash F O p t \backslash R M 062 X \backslash G e n \backslash C 7 H 5 O 2(1-) \backslash R O O T \backslash 02-J u l-2019 \backslash 0 \backslash \backslash \#$ M062X/G

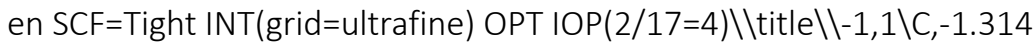
2346856,0.7793874027,1.1858781372\0,-1.4223798037,1.4530165797,2.22711 $31999 \backslash 0,-2.0761457723,0.6986527965,0.2049639799 \backslash C,-0.0351129251,-0.092$ $2885231,1.0971518028 \backslash \mathrm{C}, 0.2035932821,-0.8915213832,-0.0162578474 \backslash \mathrm{H},-0.5$ $368214094,-0.8749239964,-0.8057103759 \backslash C, 1.348507656,-1.6741588608,-0.0$ $998432044 \backslash H, 1.5218202956,-2.2921253378,-0.9731660558 \backslash \mathrm{C}, 2.2750410098,-1$ $.666575014,0.9369098482 \backslash H, 3.1686780666,-2.2757146346,0.8746507877 \backslash C, 2$. 
$0452215324,-0.8709498604,2.0539216683 \backslash \mathrm{H}, 2.7634821723,-0.8605546229,2.8$ $654077362 \backslash C, 0.8975877672,-0.0912776126,2.1293931503 \backslash H, 0.6893077196,0.5$ $385807172,2.9845727332 \backslash \mid$ Version=ES64L-G16RevB.01\State $=1-A^{\prime} \backslash H F=-420.24$ 73417 \RMSD =5.233e-09\RMSF=1.155e-04\Dipole $=2.9231135,-1.9921764,-0.203$ 0697\Quadrupole $=-6.4822301,0.8430985,5.6391316,9.1842322,0.3777822,-1$. 3217173\PG=CS [SG(C7H5O2)]入\@

\section{CARB2.xyz-M062X-MG3S}

$1 \backslash 1 \backslash G I N C-R 427 \backslash F O p t \backslash R M 062 X \backslash G e n \backslash C 7 H 6 O 2 \backslash R O O T \backslash 02-J u l-2019 \backslash 0 \backslash \backslash \#$ M062X/Gen S

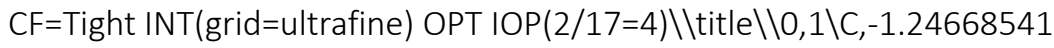
44,0.7571509767,1.2221976072\0,-1.4892445913,1.4598004369,2.1649341238 $\backslash 0,-2.0707214446,0.6698772183,0.1613717281 \backslash \mathrm{H},-2.8194480013,1.252847335$ $7,0.3386451053 \backslash C,-0.0359623234,-0.0951010814,1.0913681294 \backslash C, 0.18524591$ $37,-0.8879800547,-0.0304320776 \backslash \mathrm{H},-0.5369157065,-0.8912036002,-0.833874$ $5578 \backslash \mathrm{C}, 1.3305733789,-1.6658704265,-0.1053824854 \backslash \mathrm{H}, 1.5064360371,-2.2834$ $202193,-0.9751746935 \backslash \mathrm{C}, 2.249672054,-1.6512148754,0.935346425 \backslash \mathrm{H}, 3.14221$ $95979,-2.2591398065,0.8739757339 \backslash C, 2.0273000013,-0.8587407433,2.055163$ $1987 \backslash \mathrm{H}, 2.7446507511,-0.8493576602,2.8639037956 \backslash \mathrm{C}, 0.8848167169,-0.08025$ $38267,2.1342839326 \backslash \mathrm{H}, 0.6865590574,0.5454701299,2.9934176307 \backslash \backslash V e r s i o n=E$ S64L-G16RevB.01 \State=1-A' $\backslash H F=-420.7998628 \backslash R M S D=5.464 \mathrm{e}-09 \backslash R M S F=4.837 \mathrm{e}-$ 05\Dipole $=0.3234392,-0.5114091,-0.5238503 \backslash$ Quadrupole $=3.7125662,-2.4906$ 712,-1.221895,-2.6452922,3.6009398,-0.8869711\PG=CS [SG(C7H6O2)]\\@

\section{CARB3-BASE.xyz-M062X-MG3S}

$1 \backslash 1 \backslash G I N C-R 435 \backslash F O p t \backslash R M 062 X \backslash G e n \backslash C 2 H 2 C l 1 O 2(1-) \backslash R O O T \backslash 02-J u l-2019 \backslash 0 \backslash \backslash \#$ M062 X/Gen SCF=Tight INT(grid=ultrafine) OPT IOP(2/17=4)\\title \-1,1\H,0.4 $098252469,0.1785117582,-0.9721661982 \backslash C,-0.3131534817,-0.1704277841,-0$. $2415692055 \backslash \mathrm{H},-1.1023055192,-0.7110005605,-0.7546473156 \backslash \mathrm{Cl}, 0.5459983365$ $,-1.3792424043,0.7877409801 \backslash C,-0.9320537694,1.0615367259,0.4939505791 \backslash$ 0,-0.808859038,1.1512440912,1.7172115064\0,-1.4898987338,1.8069587707,

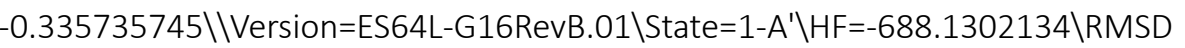
$=6.215 \mathrm{e}-09 \backslash \mathrm{RMSF}=3.917 \mathrm{e}-05 \backslash$ Dipole $=0.8958081,-1.6992299,-0.7213484 \backslash \mathrm{Quadr}$ upole $=2.9675188,-4.4678975,1.5003787,7.0360545,-1.3101553,0.8102165 \backslash P G$ $=\mathrm{CS}[\mathrm{SG}(\mathrm{C} 2 \mathrm{Cl} 1 \mathrm{O} 2), \mathrm{X}(\mathrm{H} 2)] \backslash \backslash @$

\section{CARB3.xyz-M062X-MG3S}

$1 \backslash 1 \backslash G I N C-R 436 \backslash F O p t \backslash R M 062 X \backslash G e n \backslash C 2 H 3 C l 1 O 2 \backslash R O O T \backslash 02-J u l-2019 \backslash 0 \backslash \backslash \#$ M062X/Ge

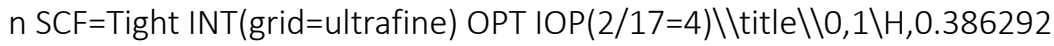
$0115,0.209958206,-0.9834730213 \backslash C,-0.3146836307,-0.1683406199,-0.243646$ $3115 \backslash \mathrm{H},-1.1208596062,-0.6766214864,-0.7666676022 \backslash \mathrm{Cl}, 0.5113123115,-1.31$ $65892907,0.8028550534 \backslash C,-0.8923472359,1.0012119005,0.5232850569 \backslash 0,-0.8$ $339654478,1.1891356218,1.6976079238 \backslash 0,-1.5048444888,1.8311891772,-0.34$ 05550977\} \backslash \mathrm { H } , - 1 . 8 6 6 2 9 2 3 5 3 8 , 2 . 5 6 9 6 6 3 4 8 6 4 , 0 . 1 6 6 6 2 5 9 1 2 6 \backslash | V e r s i o n = E S 6 4 L - G 1 6 R evB.01\State $=1-A^{\prime} \backslash H F=-688.6743083 \backslash R M S D=7.312 e-09 \backslash R M S F=6.306 e-05 \backslash$ Dipole $=-0.3985461,0.4457546,-0.9477273 \backslash$ Quadrupole $=0.8027387,1.8022804,-2.605$ 0191,-0.9294732,-0.2492433,-0.2846958\PG=CS [SG(C2H1Cl1O2),X(H2)]\\@

\section{CARB4-BASE.xyz-M062X-MG3S}

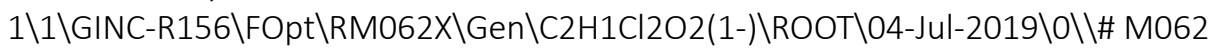
X/Gen SCF=Tight INT(grid=ultrafine) OPT IOP(2/17=4)\\title \-1,1 \Cl,1. $3392619279,-0.0150088603,-1.7644268678 \backslash \mathrm{C},-0.241536289,-0.3722140628,-0$ $.9947091666 \backslash \mathrm{H},-0.9861393033,-0.2472430911,-1.7690170113 \backslash \mathrm{Cl},-0.28612645$ $52,-2.0898809055,-0.5045631391 \backslash C,-0.4967271978,0.6323831941,0.20084427$ $84 \backslash 0,0.0598867582,0.3639611963,1.2642834738 \backslash 0,-1.2308994407,1.56652252$ 


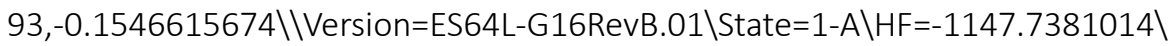
$\mathrm{RMSD}=4.707 \mathrm{e}-09 \backslash \mathrm{RMSF}=2.811 \mathrm{e}-05 \backslash \mathrm{Dipole}=0.4574734,-1.4849315,-1.6099216 \backslash \mathrm{Q}$ uadrupole $=3.5050656,-2.8147094,-0.6903562,4.2319816,1.871572,-3.489715$ $3 \backslash P G=C 01[X(C 2 \mathrm{H} 1 \mathrm{Cl} 2 \mathrm{O} 2)] \backslash \backslash @$

CARB4.XYz-M062X-MG3S

$1 \backslash 1 \backslash G I N C-R 530 \backslash F O p t \backslash R M 062 X \backslash G e n \backslash C 2 H 2 C l 2 O 2 \backslash R O O T \backslash 02-J u l-2019 \backslash 0 \backslash \backslash \#$ M062X/Ge n SCF=Tight INT(grid=ultrafine) OPT IOP(2/17=4)\|title $\backslash 0,1 \backslash C l, 1.24830$ $75795,0.0752663314,-1.8269307676 \backslash C,-0.2483542624,-0.3815417418,-0.9879$ $325189 \backslash \mathrm{H},-1.0613569625,-0.2528556744,-1.6925357122 \backslash \mathrm{Cl},-0.2192709384,-2$ $.0511669178,-0.4579943596 \backslash C,-0.410681092,0.5700252256,0.1947165762 \backslash 0,-$ $0.1048413929,0.3350872332,1.3195396506 \backslash 0,-0.9348997142,1.7242454487,-0$ $.2337445997 \backslash \mathrm{H},-0.980053217,2.330010095,0.5176617312 \backslash \backslash$ Version=ES64L-G16 RevB.01\State $=1-A \backslash H F=-1148.2695281 \backslash R M S D=2.073 e-09 \backslash R M S F=7.754 e-05 \backslash D i p o l$ $\mathrm{e}=-0.6018958,0.6721773,-0.3414546 \backslash$ Quadrupole $=0.4407989,2.7704547,-3.21$ $12537,-1.1323354,-0.5971469,2.3507738 \backslash P G=C 01$ [X(C2H2Cl2O2)]\\@

\section{CARB5-BASE.xyz-M062X-MG3S}

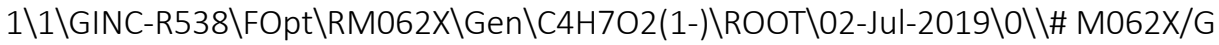
en SCF=Tight INT(grid=ultrafine) OPT IOP(2/17=4)\title \-1,1\H,1.5165 56802,-3.801678071,-1.744098267\C,1.2760206127,-2.7370375237,-1.796266 $3644 \backslash \mathrm{H}, 2.2053454149,-2.178232653,-1.6612672519 \backslash \mathrm{H}, 0.9166426438,-2.52547$ $58512,-2.8063284447 \backslash \mathrm{C}, 0.2386299006,-2.327954155,-0.7529266455 \backslash \mathrm{H},-0.675$ $9199129,-2.9097411479,-0.8793729695 \backslash H, 0.5956363588,-2.5664271719,0.250$ $3416151 \backslash \mathrm{C},-0.1077721626,-0.8490816962,-0.8124172448 \backslash \mathrm{H}, 0.7903144815,-0$. $2403147423,-0.6675423372 \backslash \mathrm{H},-0.4889252119,-0.5857032331,-1.8041262237 \backslash \mathrm{C}$ ,-1.1644220212,-0.3748493065,0.2328053124\0,-1.61198449887,-1.248986865 $9,1.0022046521 \backslash 0,-1.4412024071,0.8399724177,0.1751541691 \backslash \backslash V e r s i o n=E S 64$ L-G16RevB.01\State=1-A \HF=-307.1261659 \RMSD $=8.295 \mathrm{e}-09 \backslash \mathrm{RMSF}=4.534 \mathrm{e}-06 \backslash \mathrm{D}$ ipole $=1.9559714,-1.6824248,-1.690313 \backslash$ Quadrupole $=1.2243357,-3.0503798,1$ $.826044,5.3066751,6.6389218,-2.4461769 \backslash P G=C 01[X(C 4 H 7 O 2)] \backslash \backslash @$

\section{CARB5.xyz-M062X-MG3S}

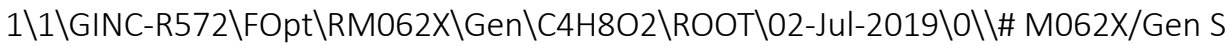
CF=Tight INT(grid=ultrafine) OPT IOP(2/17=4)\\itle $\backslash 0,1 \backslash H, 1.720344013$ $5,-3.6763324363,-1.4387756216 \backslash C, 1.3031759502,-2.7064710666,-1.70538569$ $98 \backslash \mathrm{H}, 2.1313464938,-2.0100823776,-1.8415926115 \backslash \mathrm{H}, 0.7946152935,-2.811529$ $6402,-2.6642258152 \backslash \mathrm{C}, 0.3446121809,-2.2054433877,-0.633002542 \backslash \mathrm{H},-0.4666$ $963591,-2.9223348855,-0.4934522406 \backslash H, 0.8608542574,-2.1179806058,0.3234$ $440963 \backslash \mathrm{C},-0.2513455611,-0.843475756,-1.0066155483 \backslash \mathrm{H}, 0.5510941878,-0.11$ $10240061,-1.1089040769 \backslash \mathrm{H},-0.7933906159,-0.9078041888,-1.9478075 \backslash \mathrm{C},-1.2$ $145801299,-0.3635388282,0.0411337889 \backslash 0,-2.4074269237,-0.3181108359,-0$. $0677092 \backslash 0,-0.5815515923,-0.0019902021,1.176135001 \backslash H,-1.2590611952,0.26$ $58582169,1.8098579696 \backslash \mid$ Version=ES64L-G16RevB.01 \State=1-A|HF=-307.6884 039\RMSD $=5.545 \mathrm{e}-09 \backslash \mathrm{RMSF}=1.276 \mathrm{e}-05 \backslash \mathrm{Dipole}=0.7478932,-0.1534416,0.084431$ 2\Quadrupole $=-4.350566,1.251852,3.098714,0.3429141,-1.7086748,1.201618$ $4 \backslash \mathrm{PG}=\mathrm{CO} 1[\mathrm{X}(\mathrm{C} 4 \mathrm{H} 8 \mathrm{O} 2)] \backslash \backslash @$

N1-BASE.xyz-M062X-MG3S

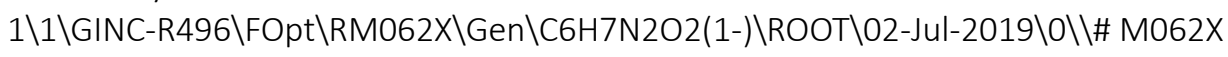
/Gen SCF=Tight INT(grid=ultrafine) OPT IOP(2/17=4)\\itle \-1,1\N,0.76 98626207,-0.5173227982,0.4292275574\C,1.9934844088,-1.0359529229,0.090 $7139597 \backslash \mathrm{N}, 2.7368426183,-1.5525798904,1.1286012694 \backslash \mathrm{C}, 2.3100946566,-1.57$ $16250209,2.4807625127 \backslash C, 1.0178278147,-0.9995624468,2.6896043287 \backslash C, 0.21$ 
$57666481,-0.4650365023,1.7105258068 \backslash C, 0.0135917111,0.0185368348,-0.690$ $8101521 \backslash 0,2.4196138641,-1.0488739434,-1.0633929862 \backslash C, 4.0313325913,-2.1$ $02409551,0.7769954179 \backslash 0,3.055760185,-2.0587805568,3.3303168081 \backslash H, 0.681$ 2056269,-1.0082855268,3.7180743998\\,-0.1754019534,-0.75388722234,-1.43 $84018923 \backslash \mathrm{H}, 0.5631200336,0.8231960608,-1.1825549642 \backslash \mathrm{H},-0.9189017564,0.3$ $884526668,-0.2790720673 \backslash \mathrm{H}, 4.4868132304,-2.4634238381,1.6923791989 \backslash \mathrm{H}, 4$. $6527993274,-1.3371351338,0.3127309366 \backslash H, 3.9143683729,-2.9134202078,0.0$ 586498659 \|Version=ES64L-G16RevB.01\State $=1-A \backslash H F=-492.8156028 \backslash R M S D=8.4$ 67e-09\RMSF $=3.331$ e-05\Dipole $=0.7890523,-0.1508612,-1.3519326 \backslash$ Quadrupol $\mathrm{e}=0.1010067,7.4724759,-7.5734826,4.3164722,1.531869,2.1020982 \backslash \mathrm{PG}=\mathrm{C0}$ [ X(C6H7N2O2)]\\@

N1.xyz-M062X-MG3S

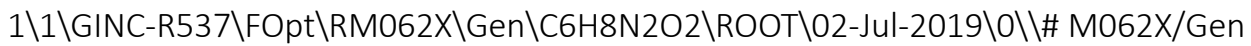

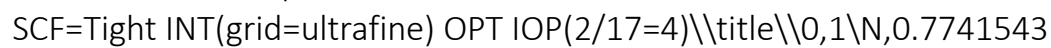

$167,-0.4974647916,0.412928146 \backslash C, 2.0097178446,-1.0372387375,0.087271934$ $5 \backslash \mathrm{N}, 2.7434259607,-1.5521283535,1.1374880245 \backslash \mathrm{C}, 2.3365741201,-1.56903219$ ,2.4850009194\C,1.0302615624,-0.9811327443,2.7226275783\C,0.3217300076 $,-0.4825050126,1.7018904873 \backslash C,-0.0027277117,0.0475543132,-0.6917352044$ $\backslash 0,2.4082813991,-1.0490458615,-1.0561718234 \backslash C, 4.0413149142,-2.11673224$ $59,0.7795796636 \backslash 0,3.0427816732,-2.0421638572,3.3460950322 \backslash \mathrm{H}, 0.66322370$ $22,-0.9640603755,3.7346145134 \backslash \mathrm{H},-0.6538433272,-0.0376618909,1.84086416$ $23 \backslash \mathrm{H},-0.1993058347,-0.7277038673,-1.428719763 \backslash \mathrm{H}, 0.5493578944,0.8486098$ $423,-1.1779953022 \backslash \mathrm{H},-0.9395284526,0.429760736,-0.2973676717 \backslash \mathrm{H}, 4.504574$ $9728,-2.4748223894,1.6907253203 \backslash \mathrm{H}, 4.6553835287,-1.3525571944,0.3091482$ $117 \backslash \mathrm{H}, 3.9039634296,-2.9306353799,0.0716157711 \backslash \backslash V e r s i o n=E S 64 L-G 16 R e v B .0$ $1 \backslash$ State $=1-A \backslash H F=-493.4115011 \backslash R M S D=6.178 e-09 \backslash R M S F=6.097 e-06 \backslash$ Dipole $=-1.42$ $54654,0.7462372,-0.406305 \backslash$ Quadrupole $=6.5878682,1.4911145,-8.0789827,-2$ .7056349,-1.2792492,1.9575596\PG=C01 [X(C6H8N2O2)]\\@

N2-BASE.xyz-M062X-MG3S

$1 \backslash 1 \backslash G I N C-R 538 \backslash F O p t \backslash R M 062 X \backslash G e n \backslash C 10 H 10 N 101(1-) \backslash R O O T \backslash 02-J u l-2019 \backslash 0 \backslash \backslash \#$ M06 2X/Gen SCF=Tight INT(grid=ultrafine) OPT IOP(2/17=4)\\title \-1,1\C,2.

$985443915,-0.7574542748,0.5128520272 \backslash C, 3.1501375215,-0.6984452313,1.88$ $70373008 \backslash C, 2.0471684423,-0.4123704816,2.6962330264 \backslash C, 0.8091496353,-0.2$ $066003896,2.1286565985 \backslash C, 1.7398308507,-0.5487633462,-0.0798042847 \backslash C, 0$. $6370183159,-0.2995260846,0.7340902059 \backslash \mathrm{C},-0.5226747354,0.0881569178,2.7$ $639702962 \backslash \mathrm{N},-0.6941865158,-0.0072460058,0.4339746093 \backslash \mathrm{C},-1.296341242,0$. $6949680836,1.5944270898 \backslash \mathrm{H}, 3.8401057074,-0.9508831423,-0.1238023932 \backslash \mathrm{H}, 4$ $.1271044537,-0.8536144205,2.3263288306 \backslash \mathrm{H}, 2.1618391499,-0.3453956848,3$. $7721745863 \backslash \mathrm{H}, 1.6674905531,-0.5573076985,-1.1544683577 \backslash \mathrm{H},-0.4267356867$, $0.7591310963,3.6199915878 \backslash \mathrm{C},-1.3778000828,-0.2661328112,-0.7025736192 \backslash$ $\mathrm{H},-2.3601302076,0.4706074957,1.5995122064 \backslash 0,-2.5628726035,0.0450402515$ $,-0.8303884982 \backslash \mathrm{C},-0.6792898565,-0.9677654327,-1.8558177811 \backslash \mathrm{H},-1.464622$ $9876,-1.3326210845,-2.5121728074 \backslash \mathrm{H},-0.0539097776,-1.7989009247,-1.5341$ $514788 \backslash \mathrm{H},-0.0578319386,-0.2700368722,-2.4221635647 \backslash \mathrm{H},-0.9202429107,-0$. 8748699596,3.1620444198\|Version=ES64L-G16RevB.01 \State=1-A \HF=-516.98 92057 \RMSD $=5.899 \mathrm{e}-09 \backslash \mathrm{RMSF}=2.319 \mathrm{e}-05 \backslash$ Dipole $=2.6929553,-1.6857729,-0.250$ 713\Quadrupole=-9.2730674,2.0767162,7.1963511,6.6991686,-5.3157674,-0. $6651935 \backslash P G=C 01[X(C 10 H 10 N 101)] \backslash \backslash$

N2.xyz-M062X-MG3S

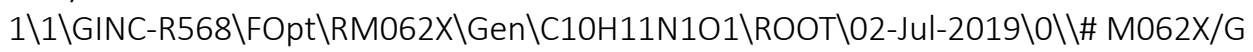
en SCF=Tight INT(grid=ultrafine) OPT IOP(2/17=4)\\title \0,1\C,2.96930 
89859,-0.8943215248,0.5883121613\C,3.110929916,-0.8059854572,1.9633807 $055 \backslash C, 2.0102181618,-0.4731128461,2.7493321207 \backslash C, 0.79226854,-0.24364868$ $82,2.1465056629 \backslash C, 1.7452090132,-0.6614795884,-0.0343997201 \backslash C, 0.6495419$ $494,-0.3461296365,0.7587963919 \backslash C,-0.5329086531,0.1041888342,2.77225415$ $84 \backslash \mathrm{N},-0.6801868055,-0.0446083421,0.4066966192 \backslash \mathrm{C},-1.3828968427,0.527957$ $7986,1.5670982071 \backslash \mathrm{H}, 3.8261090975,-1.1383184686,-0.0247831041 \backslash \mathrm{H}, 4.07265$ $05278,-0.9851124806,2.422638403 \backslash \mathrm{H}, 2.107438987,-0.3914262065,3.82441323$ $9 \backslash \mathrm{H}, 1.683914508,-0.7134978252,-1.1078764124 \backslash \mathrm{H},-0.4530202295,0.89429449$ $74,3.5158254206 \backslash \mathrm{C},-1.3610601713,-0.1460038026,-0.7791635686 \backslash \mathrm{H},-2.40224$ $17354,0.1557981441,1.58902585 \backslash 0,-2.4927605612,0.2863725896,-0.86580484$ $73 \backslash \mathrm{C},-0.6873828267,-0.8239473499,-1.9452160616 \backslash \mathrm{H},-0.1846813477,-1.7414$ $279576,-1.6454728147 \backslash \mathrm{H}, 0.0472295405,-0.1570021131,-2.3962641254 \backslash \mathrm{H},-1.4$ $573018011,-1.0401640973,-2.6785865535 \backslash \mathrm{H},-0.9594755018,-0.7722607075,3$. $2638999605 \backslash \mathrm{H},-1.425372751,1.6132952284,1.4600683077 \backslash \backslash V e r s i o n=E S 64 L-G 16$ RevB.01\State $=1-A \backslash H F=-517.6324872 \backslash$ RMSD $=4.056 e-09 \backslash R M S F=8.490 \mathrm{e}-06 \backslash$ Dipole $=1.0041408,-0.3024615,0.5301144 \backslash$ Quadrupole $=-5.0944437,-0.8564173,5.950$ 8609,1.1123698,-5.2657888,2.8358767\PG=C01 [X(C10H11N1O1)]\\@

N3-BASE.xyz-M062X-MG3S

$1 \backslash 1 \backslash G I N C-R 471 \backslash F O p t \backslash R M 062 X \backslash G e n \backslash C 2 H 5(1-) \backslash R O O T \backslash 12-A u g-2019 \backslash 0 \backslash \backslash \#$ M062X/Gen SCF=Tight INT(grid=ultrafine) OPT IOP(2/17=4)\title \-1,1\H,-0.24180 $56859,2.628033092,0.7419585028 \backslash C, 0.0814444766,1.726308513,1.2833223385$ $\backslash \mathrm{H}, 1.1740727063,1.7906739033,1.3959870393 \backslash \mathrm{C},-0.3004844268,0.4680490388$ $, 0.4991818584 \backslash \mathrm{H},-1.3894943595,0.3868145788,0.391204692 \backslash \mathrm{H}, 0.1053380715$, $0.348630694,-0.5322583879 \backslash \mathrm{H}, 0.006219361,-0.4386190991,1.035918678 \backslash \backslash \mathrm{Ver}$

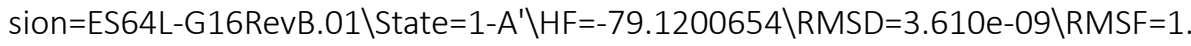
382e-04\Dipole $=-0.0118573,-1.0398055,-1.3056038 \backslash$ Quadrupole $=2.5263603,0$ .20563,-2.7319904,-0.4562587,1.035055,-2.9411282\PG=CS [SG(C2H1),X(H4) ]\@

N3.xyz-M062X-MG3S

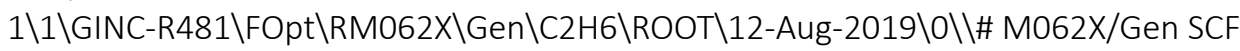
$=$ Tight INT(grid=ultrafine) OPT IOP(2/17=4)\\title $\backslash 0,1 \backslash H,-0.2402141658$

,2.6072056782,0.7286413555\C,0.0846730797,1.7129160716,1.2589700529\H, $1.1655721874,1.7756326738,1.3784602069 \backslash \mathrm{H},-0.3585327297,1.7409265553,2$. $253624854 \backslash \mathrm{C},-0.316804046,0.4486768828,0.5096595104 \backslash \mathrm{H},-1.3977251563,0.3$ $857988007,0.3904535947 \backslash \mathrm{H}, 0.1261397097,0.4208213807,-0.485116369 \backslash \mathrm{H}, 0.00$ 83672556,-0.4456062254,1.0398250478\|Version=ES64L-G16RevB.01 \HF=-79.8 $022655 \backslash R M S D=4.835 e-09 \backslash R M S F=2.072 e-04 \backslash$ Dipole=0.,0.,0. $\backslash$ Quadrupole $=0.1257$ $287,-0.1692864,0.0435577,-0.1041939,-0.0617554,-0.1944658 \backslash P G=D 03$ [C3(C 1.C1),X(H6)]\\@

N4-BASE.xyz-M062X-MG3S

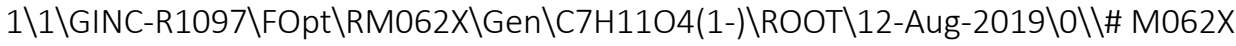
/Gen SCF=Tight INT(grid=ultrafine) OPT IOP(2/17=4)\title \-1,1\0,2.33 $70704074,0.5557882011,1.625176044 \backslash C, 2.7669498709,1.3506479692,0.548874$ $579 \backslash C, 3.0093863011,2.7488854543,1.0826571032 \backslash C, 2.0638346625,-0.7875913$ $425,1.3001891931 \backslash 0,2.2203840505,-1.1365635973,0.1397738605 \backslash C, 1.6434329$ $432,-1.4838224025,2.4502293399 \backslash \mathrm{H}, 1.5882964524,-0.9330945804,3.37432530$ $77 \backslash C, 1.2798710046,-2.8447237367,2.5322365679 \backslash 0,0.9141609807,-3.4416815$ $811,3.5442132124 \backslash 0,1.3545686641,-3.538318565,1.3437141388 \backslash C, 0.99406104$ $18,-4.8970041783,1.4133765285 \backslash \mathrm{C}, 1.1428799039,-5.4694156,0.0180198893 \backslash \mathrm{H}$ $,-0.0321043371,-4.999641442,1.7763684367 \backslash \mathrm{H}, 1.6327396013,-5.4234235635$, 2.127728469\} \mathrm { H } , 0 . 4 9 8 5 1 5 2 3 8 9 , - 4 . 9 3 5 4 9 0 5 4 0 6 , - 0 . 6 7 9 7 2 7 3 8 7 2 \backslash \mathrm { H } , 0 . 8 7 6 3 9 1 0 7 4 9 \text { , } 
$-6.5277356954,0.0061959436 \backslash H, 2.1706240723,-5.3604596118,-0.3269504678 \backslash$ $\mathrm{H}, 2.0137938532,1.3586225425,-0.2438763877 \backslash \mathrm{H}, 3.6777122283,0.9356835035$, $0.1083117041 \backslash \mathrm{H}, 3.7681722878,2.7307024041,1.8650666604 \backslash \mathrm{H}, 2.093375199,3$. $1564786675,1.5105070772 \backslash H, 3.346984498,3.412347695,0.2847001874 \backslash \backslash V e r s i o$ $\mathrm{n}=\mathrm{ES} 64 \mathrm{~L}-\mathrm{G} 16 \mathrm{RevB} .01 \backslash \mathrm{State}=1-\mathrm{A} \backslash \mathrm{HF}=-574.2972619 \backslash \mathrm{RMSD}=6.007 \mathrm{e}-09 \backslash \mathrm{RMSF}=6.692$ e-06\Dipole $=0.3510579,0.4634546,-1.1036694 \backslash$ Quadrupole $=6.0682465,-0.998$ 3077,-5.0699387,-3.0546348,3.7185216,4.0634448\PG=C01 [X(C7H11O4)]\\@

N4.xyz-M062X-MG3S

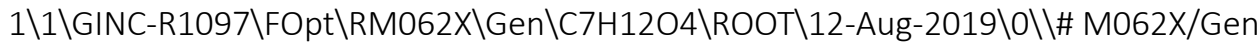
SCF=Tight INT(grid=ultrafine) OPT IOP(2/17=4)\\title \0,1\0,2.4147057 $156,0.4991228,1.6486497627 \backslash C, 2.3392701236,1.4660612447,0.5897428823 \backslash C$, $3.2467594572,2.6137751219,0.9588156234 \backslash C, 1.6602413597,-0.5915426544,1$. 49210563\0,0.9582418196,-0.7939376287,0.5430521281\C,1.8081992228,-1.5 $176351648,2.6796216956 \backslash \mathrm{H}, 2.8637667308,-1.7550668522,2.804177265 \backslash \mathrm{H}, 1.45$ $65914557,-1.0027877725,3.5709530322 \backslash C, 0.9939845041,-2.7695785334,2.471$ $1513015 \backslash 0,-0.0697558256,-2.979731956,2.9806546663 \backslash 0,1.6102427348,-3.60$ $98390054,1.6395114483 \backslash C, 0.8741622305,-4.7898563809,1.2882282063 \backslash C, 1.71$ $39806851,-5.5730157956,0.3092229389 \backslash \mathrm{H},-0.0795802053,-4.4838546611,0.85$ 90593996\H,0.666459276,-5.3546941147,2.1969914539\H,1.912229092,-4.981 $3913945,-0.5827285017 \backslash \mathrm{H}, 1.1876120468,-6.4795435949,0.0142756132 \backslash \mathrm{H}, 2.66$ $50923549,-5.8561129807,0.7573112061 \backslash \mathrm{H}, 1.3016663298,1.7806083238,0.4802$ $604787 \backslash \mathrm{H}, 2.6410650985,0.9857911274,-0.3407843661 \backslash \mathrm{H}, 4.2742368432,2.2716$ $512518,1.0702862131 \backslash \mathrm{H}, 2.9293731231,3.0693084065,1.8952396178 \backslash \mathrm{H}, 3.21743$ 58269,3.3720602136,0.1778723051 \\Version=ES64L-G16RevB.01\State=1-A\HF $=-574.8579142 \backslash$ RMSD $=7.957 \mathrm{e}-09 \backslash \mathrm{RMSF}=7.738 \mathrm{e}-06 \backslash \mathrm{Dipole}=1.0801327,0.3126064$ ,$-0.1899323 \backslash$ Quadrupole $=-5.7199113,8.8778532,-3.1579419,0.8358224,1.653$ $3667,0.8694586 \backslash P G=C 01[X(C 7 H 12 O 4)] \backslash \backslash @$

N5-BASE.xyz-M062X-MG3S

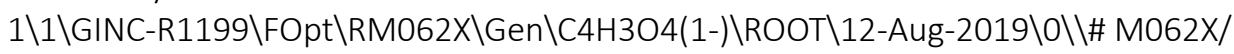
Gen SCF=Tight INT(grid=ultrafine) OPT IOP(2/17=4)\title \\-1,1\0,-0.48 $43240272,-0.1889611289,1.2306248947 \backslash C, 0.2959162957,0.8436862549,0.7100$ $288303 \backslash 0,0.0729705032,1.0746221862,-0.6474942656 \backslash \mathrm{H}, 0.0240193595,1.7538$ $484492,1.2413611565 \backslash \mathrm{H}, 1.3566706606,0.6033392578,0.8629823389 \backslash \mathrm{C}, 0.28937$ 48459,-0.0600142438,-1.4403748027\C,0.2268052946,-1.2886989442,-0.7454 $587562 \backslash 0,0.481123529,0.1392246393,-2.6263262794 \backslash C,-0.3039971521,-1.405$ $3731164,0.5587281477 \backslash \mathrm{H}, 0.3737828011,-2.1938366099,-1.3102867971 \backslash 0,-0.6$ 416921104,-2.4090967442,1.1601355329 \|Version=ES64L-G16RevB.01\State=1 $-A \backslash H F=-455.195381 \backslash R M S D=7.121 e-09 \backslash R M S F=7.520 e-05 \backslash$ Dipole $=0.4208801,1.075$ 4837,0.8477638\Quadrupole=7.0323965,-0.1410432,-6.8913533,-2.1555047,4 .8867778,8.9147381\PG=C01 [X(C4H3O4)]\\@

N5.xyz-M062X-MG3S

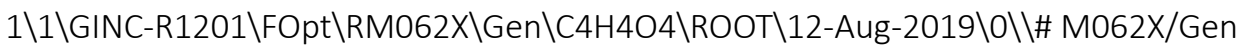

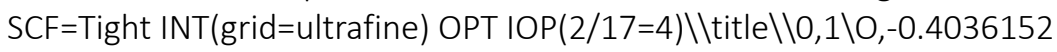
$542,-0.2713604281,1.2105454584 \backslash \mathrm{C}, 0.4416083077,0.712418956,0.6640477595$ $\backslash 0,0.1286644567,0.9945976355,-0.6788422769 \backslash \mathrm{H}, 0.2701675878,1.6224966127$ $, 1.2255258273 \backslash \mathrm{H}, 1.4845448799,0.3967179798,0.7464429903 \backslash \mathrm{C}, 0.0773618223$, $-0.0700246973,-1.5164159624 \backslash C, 0.2192116688,-1.4056492148,-0.8178231792$ $\backslash 0,-0.0803367943,0.0745866198,-2.6837765768 \backslash \mathrm{C},-0.4949396416,-1.4324647$ $502,0.5167943239 \backslash \mathrm{H},-0.1467994774,-2.2021653939,-1.4545732189 \backslash \mathrm{H}, 1.28513$ $79757,-1.5740026844,-0.6306602353 \backslash 0,-1.1017655316,-2.3575906352,0.9459$ $550902 \backslash \backslash$ Version=ES64L-G16RevB.01 \State=1-A \HF=-455.7267509\RMSD $=4.956 \mathrm{e}$ 
-09\RMSF $=3.640$ e-05\Dipole $=1.2533672,0.300869,0.5548327 \backslash$ Quadrupole $=4.04$ 06128,0.775482,-4.8160949,-1.8678146,2.7368204,6.7812057\PG=C01 [X(C4H 404)]\\@

P1-BASE.xyz-M062X-MG3S

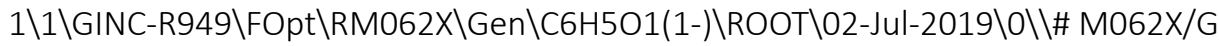
en SCF=Tight INT(grid=ultrafine) OPT IOP(2/17=4)\title \-1,1\H,-1.496 1640669,1.1699189745,-1.7319411513\C,-2.3464930457,1.5068347664,-1.153 9162746\C,-3.0410785008,0.6364272211,-0.3101178085\Н,-2.7191944009,-0. $3986955269,-0.2344985084 \backslash \mathrm{C},-4.1275804591,1.0546456316,0.4324310208 \backslash \mathrm{H},-$ 4.6519142874,0.3616997541,1.0810455125\C,-4.6206797528,2.4079088617,0. $3919989227 \backslash 0,-5.6109023872,2.8002530618,1.0651187744 \backslash C,-3.8762762196,3$ $.2708509863,-0.4896457377 \backslash \mathrm{H},-4.2051258764,4.3018436631,-0.5582949558 \backslash \mathrm{C}$ $,-2.7924380408,2.8291411994,-1.22242072 \backslash \mathrm{H},-2.2735743973,3.5311444151,-$ 1.8695518971 \|Version=ES64L-G16RevB.01\State=1-A1\HF=-306.8766291\RMSD $=2.791 \mathrm{e}-09 \backslash \mathrm{RMSF}=2.046 \mathrm{e}-05 \backslash$ Dipole $=1.3925306,-0.5517459,-0.9465952 \backslash \mathrm{Quadr}$ upole $=-4.150067,3.9612573,0.1888097,3.187647,5.0380981,-2.7709113 \backslash P G=C$ 02V [C2(H1C1C1O1),SGV(C4H4)]\\@

P1.xyz-M062X-MG3S

$1 \backslash 1 \backslash G I N C-R 951 \backslash F O p t \backslash R M 062 X \backslash G e n \backslash C 6 H 601 \backslash R O O T \backslash 02-J u l-2019 \backslash 0 \backslash \backslash \#$ M062X/Gen S

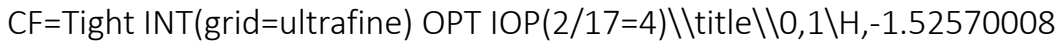
99,1.1745322462,-1.709562374\C,-2.3746562471,1.51264222225,-1.133035223 $4 \backslash \mathrm{C},-3.051072912,0.6330574444,-0.3009463056 \backslash \mathrm{H},-2.7315154347,-0.3974713$ $448,-0.2249366723 \backslash \mathrm{C},-4.1417081468,1.0629784278,0.4411462205 \backslash \mathrm{H},-4.66946$ $92233,0.3746416003,1.0910351153 \backslash C,-4.5599905878,2.3850994441,0.3503422$ $134 \backslash 0,-5.6218336929,2.8645381239,1.0530982964 \backslash \mathrm{H},-6.002839802,2.1644971$ $313,1.5881619919 \backslash \mathrm{C},-3.8883642304,3.2735181161,-0.4807404512 \backslash \mathrm{H},-4.23155$ $58379,4.2971390291,-0.5354056653 \backslash C,-2.8010830948,2.8326016012,-1.21655$ 48597\\, -2.2823798932,3.5288502951,-1.8616889156|\Version=ES64L-G16Rev B.01\State $=1-A^{\prime} \backslash H F=-307.4438323 \backslash R M S D=6.485 e-09 \backslash R M S F=1.736 e-04 \backslash$ Dipole $=-$ $0.0361767,-0.4832632,0.1860143 \backslash$ Quadrupole $=-0.2384062,0.5610032,-0.3225$ 97,1.5470952,-3.7820139,-2.8263011\PG=CS [SG(C6H6O1)]\\@

P2-BASE.xyz-M062X-MG3S

$1 \backslash 1 \backslash G I N C-R 957 \backslash F O p t \backslash R M 062 X \backslash G e n \backslash C 6 H 4 N 1 O 3(1-) \backslash R O O T \backslash 02-J u l-2019 \backslash 0 \backslash \backslash \#$ M062X

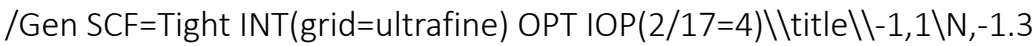

$385785468,2.6136534244,1.3224902596 \backslash C,-1.7153216457,1.2561896358,1.236$ $0855085 \backslash \mathrm{C},-3.0580264112,0.9075829809,0.9997303038 \backslash \mathrm{H},-3.7817857232,1.70$ $2758482,0.8880042979 \backslash \mathrm{C},-3.42802402,-0.4020258845,0.9152481456 \backslash \mathrm{H},-4.459$ 4077117,-0.6762043794,0.7335924706\C,-2.4775812856,-1.4903500134,1.061 $2638472 \backslash 0,-2.8093620145,-2.685807413,0.9851710631 \backslash \mathrm{C},-1.1085562374,-1.0$ $704571768,1.3032799819 \backslash \mathrm{H},-0.3708534234,-1.8544564107,1.4175809443 \backslash \mathrm{C},-0$ $.750662371,0.242639782,1.385737263 \backslash \mathrm{H}, 0.2745872393,0.5337805632,1.56660$ 90456\0,-0.1568928397,2.8975509619,1.5301361796\0,-2.199326153,3.48614 $56168,1.1884504037 \backslash \mid V e r s i o n=E S 64 L-G 16 R e v B .01 \backslash S t a t e=1-A 1 \backslash H F=-511.420773$ $\backslash \mathrm{RMSD}=4.681 \mathrm{e}-09 \backslash \mathrm{RMSF}=4.138 \mathrm{e}-05 \backslash \mathrm{Dipole}=0.1455603,0.5244764,0.0333838 \backslash \mathrm{Qu}$ adrupole $=9.4241874,-21.7090112,12.2848238,-9.3568599,-0.6665144,-2.152$ 7974\PG=C02V [C2(N1C1C1O1),SGV(C4H4O2)]\\@

P2.xyz-M062X-MG3S

$1 \backslash 1 \backslash G I N C-R 957 \backslash F O p t \backslash R M 062 X \backslash G e n \backslash C 6 H 5 N 1 O 3 \backslash R O O T \backslash 02-J u l-2019 \backslash 0 \backslash \backslash \#$ M062X/Gen SCF=Tight INT(grid=ultrafine) OPT IOP(2/17=4)\title \0,1\N,-1.339209

$8479,2.6285397892,1.3226414816 \backslash C,-1.7291890808,1.2171996105,1.23309977$ 
42\C,-3.0557576819,0.902300445,1.0000470727\\,-3.7803395157,1.69445871 $48,0.8881269464 \backslash \mathrm{C},-3.421779545,-0.4279572899,0.915916516 \backslash \mathrm{H},-4.45461823$ $89,-0.697241271,0.7340820621 \backslash C,-2.45893676,-1.4235010949,1.0655493187 \backslash$ O,-2.7597778291,-2.7384479793,0.9928672011 \H,-3.6983129744,-2.86266298 $78,0.8295564536 \backslash \mathrm{C},-1.1267633005,-1.0882845973,1.2998894619 \backslash \mathrm{H},-0.401216$ $9414,-1.8806983282,1.4119713119 \backslash \mathrm{C},-0.7589885105,0.2386874466,1.3842689$ $432 \backslash H, 0.2649360463,0.5287142043,1.5649018736 \backslash 0,-0.1711637692,2.8758055$ $214,1.5273663574 \backslash 0,-2.2090037203,3.4611376616,1.1864049363 \backslash \backslash V e r s i o n=E S$ 64L-G16RevB.01\State=1-A' $\backslash H F=-511.950369 \backslash R M S D=4.295 e-09 \backslash R M S F=6.618 \mathrm{e}-05$ |Dipole $=-1.0288835,-1.7783192,-0.2052185 \backslash$ Quadrupole $=6.4225721,-6.40794$ 52,-0.0146268,1.1275131,1.1590125,0.0950641\PG=CS [SG(C6H5N1O3)]\\@

P3-BASE.xyz-M062X-MG3S

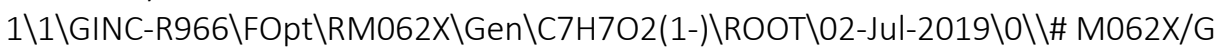
en SCF=Tight INT(grid=ultrafine) OPT IOP(2/17=4)\\title \-1,1\0,1.1103 $049816,-2.4025316723,2.5616441851 \backslash C, 0.6267999393,-1.0899576932,2.52511$ $16183 \backslash \mathrm{C},-0.7095788155,-0.8204667321,2.7970742058 \backslash \mathrm{H},-1.3687523156,-1.65$ $07847736,3.0332015985 \backslash \mathrm{C},-1.1970262777,0.4731821524,2.7567493802 \backslash \mathrm{H},-2.2$ $42860629,0.670359298,2.9617745814 \backslash C,-0.3689895021,1.6084332301,2.44199$ $91602 \backslash 0,-0.8052655609,2.7913035645,2.4063780906 \backslash C, 1.0023058639,1.26673$ $87695,2.1662715592 \backslash H, 1.6696140956,2.0818521499,1.911427334 \backslash \mathrm{C}, 1.4732255$ $711,-0.0328748263,2.2110165788 \backslash \mathrm{H}, 2.514494679,-0.2496696467,1.990805488$ $9 \backslash \mathrm{C}, 1.5848135225,-2.7687415552,3.8318044772 \backslash \mathrm{H}, 1.9404979803,-3.79885731$

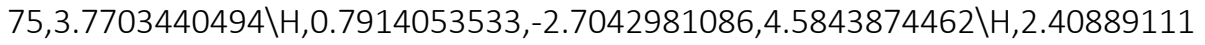
41,-2.1202968389,4.1485502459||Version=ES64L-G16RevB.01\State=1-A\HF=421.3916173\RMSD=3.298e-09\RMSF=2.971e-05\Dipole=1.1950759,-2.7322166, $0.7765256 \backslash$ Quadrupole $=6.4542413,-14.0623514,7.6081101,8.4583004,-0.4257$ 886,-0.3147947\PG=C01 [X(C7H7O2)]\\@

P3.xyz-M062X-MG3S

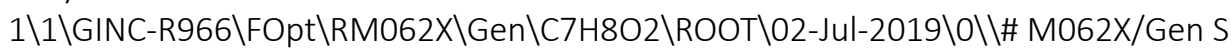
CF=Tight INT(grid=ultrafine) OPT IOP(2/17=4)\|title \} \backslash 0 , 1 \backslash 0 , 0 . 5 7 7 4 0 0 1 0 2 $,-2.366082375,3.3595129362 \backslash C, 0.3277067466,-1.0767570629,3.0005884981 \backslash C$ $,-1.0054266714,-0.7524197147,2.7672756328 \backslash \mathrm{H},-1.7531093485,-1.524905167$ $6,2.8808574988 \backslash \mathrm{C},-1.3591193255,0.5317638219,2.398344536 \backslash \mathrm{H},-2.400136182$ $4,0.7735088936,2.2189214828 \backslash C,-0.3839883858,1.5140274896,2.2566908094 \backslash$ O,-0.6780555864,2.7976028455,1.8947945809\H,-1.6241147484,2.8908918974 ,1.7657465704\C,0.9420175152,1.1947772279,2.4879081395\Н,1.6924034439, $1.9647120773,2.3752894745 \backslash \mathrm{C}, 1.3021702983,-0.0955795925,2.8590169725 \backslash \mathrm{H}$, $2.3443200812,-0.3168712802,3.0331411725 \backslash C, 1.9163879365,-2.724775081,3$. $6020419817 \backslash \mathrm{H}, 1.9076892034,-3.7767749839,3.8708724458 \backslash \mathrm{H}, 2.3391438679,-2$ $.1443172498,4.4260683595 \backslash \mathrm{H}, 2.5323210536,-2.5842417455,2.7101789086 \backslash \backslash V e$ rsion=ES64L-G16RevB.01 \State=1-A \HF=-421.9616617\RMSD=3.090e-09 \RMSF=3 .229e-05\Dipole $=-0.0073918,-0.2005805,0.0512645 \backslash$ Quadrupole $=6.4379832,-$ $2.3988352,-4.039148,-5.1852917,2.5709901,-1.1470676 \backslash P G=C 01$ [X(C7H8O2)] II@

P4-BASE.xyz-M062X-MG3S

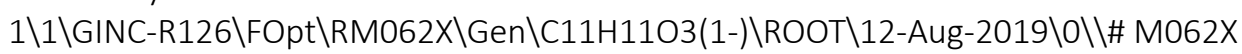

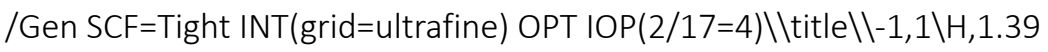
24111422,0.6143943907,-1.8466061711 \C,0.8599616796,0.5376615506,-0.908 $8751024 \backslash C,-0.5627835991,0.4516132001,-0.9481330006 \backslash C,-1.3068150447,0.4$ $620942557,-2.1542448689 \backslash \mathrm{C},-1.2322810932,0.3524002003,0.2879916583 \backslash \mathrm{H},-2$ $.316274517,0.2858989477,0.2770961004 \backslash C,-0.5664983112,0.3376057917,1.48$ 
$35111065 \backslash H,-1.0989586608,0.2607412415,2.4227616499 \backslash \mathrm{C}, 0.8687949664,0.42$ $23038108,1.5709266519 \backslash C, 1.5445900755,0.5248599268,0.2689132514 \backslash 0,2.902$ $9586201,0.6039495684,0.3714526258 \backslash 0,1.4996977387,0.4115829552,2.636683$ $513 \backslash C, 3.6251025738,0.7044366828,-0.82030491 \backslash H, 4.6760880355,0.756493573$ $8,-0.5443743022 \backslash \mathrm{H}, 3.3535239331,1.6046291705,-1.3821935398 \backslash \mathrm{H}, 3.46301346$ $08,-0.1660372722,-1.4651489697 \backslash C,-0.8664537651,0.5496499853,-3.4443656$ $871 \backslash \mathrm{H},-2.3819575161,0.3888043753,-2.0068443353 \backslash \mathrm{C},-1.7337784926,0.54992$ $65225,-4.5956113491 \backslash \mathrm{H}, 0.1890073849,0.6255549398,-3.6739690904 \backslash 0,-1.307$ $3149663,0.629957946,-5.743933192 \backslash C,-3.2419565504,0.44702566,-4.3886800$ $563 \backslash \mathrm{H},-3.7197399494,0.4628551144,-5.3643426594 \backslash \mathrm{H},-3.6122436853,1.27876$ $5417,-3.7886917431 \backslash \mathrm{H},-3.5037534594,-0.4757879547,-3.8702675798 \backslash \backslash V e r s i o$ $\mathrm{n}=\mathrm{ES} 64 \mathrm{~L}-\mathrm{G} 16 \mathrm{RevB} .01 \backslash$ State=1-A $\backslash \mathrm{HF}=-651.4534664 \backslash \mathrm{RMSD}=7.428 \mathrm{e}-09 \backslash \mathrm{RMSF}=5.343$ e-06\Dipole $=-0.5894539,0.0083244,-0.9585287 \backslash$ Quadrupole $=17.9738319,18.7$ 939311,-36.767763,0.8455813,-19.1835913,1.4131217\PG=C01 [X(C11H11O3)] 11@

\section{P4.xyz-M062X-MG3S}

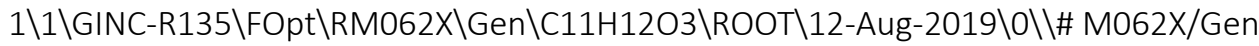

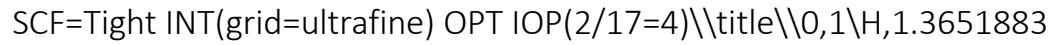
$395,0.5882082447,-1.8555118394 \backslash C, 0.8342178382,0.5042647152,-0.91942757$ $31 \backslash C,-0.5678338043,0.4247033373,-0.9161945986 \backslash C,-1.3528318726,0.451197$ $9358,-2.1479637651 \backslash \mathrm{C},-1.228447806,0.317152193,0.3022637525 \backslash \mathrm{H},-2.308709$ $1276,0.2552326203,0.315443925 \backslash C,-0.5281386213,0.2877565618,1.501468247$ $9 \backslash \mathrm{H},-1.0360746653,0.2042540732,2.4517259161 \backslash \mathrm{C}, 0.8486708924,0.366263302$ $6,1.492103921 \backslash \mathrm{C}, 1.5318967893,0.475474021,0.2668661536 \backslash 0,2.8848734813,0$ $.5447160583,0.3957399704 \backslash 0,1.5406542432,0.3388267843,2.6498000212 \backslash \mathrm{H}, 2$. $4805916504,0.4035181874,2.4459246012 \backslash C, 3.6510488856,0.654841109,-0.785$ $8104376 \backslash H, 4.6899220853,0.695194281,-0.4746683763 \backslash H, 3.3958757931,1.5661$ 444472,-1.3298781735\H,3.4951965949,-0.2116835487,-1.4311320878\C,-0.8 $941632159,0.5491952782,-3.3992186069 \backslash \mathrm{H},-2.4255325975,0.3817637341,-1.9$ $965176531 \backslash \mathrm{C},-1.7547911561,0.5695708518,-4.5996931329 \backslash \mathrm{H}, 0.1630696044,0$. $6217940921,-3.6224230738 \backslash 0,-1.2529030987,0.6610534921,-5.697974403 \backslash C,-$ $3.2556389148,0.4754058755,-4.4368723537 \backslash \mathrm{H},-3.7139206463,0.5060223306,-$ $5.4202656312 \backslash \mathrm{H},-3.6289308381,1.3032350018,-3.8335649877 \backslash \mathrm{H},-3.529899832$ $9,-0.45231498,-3.9342098151 \backslash \mid$ Version=ES64L-G16RevB.01\State=1-A $\mid H F=-65$ 1.9979858\RMSD $=7.602 \mathrm{e}-09 \backslash \mathrm{RMSF}=9.323 \mathrm{e}-06 \backslash$ Dipole $=0.4527576,-0.0302431,0$. 9600383\Quadrupole=17.5610049,-0.174243,-17.3867619,1.0758588,-1.33872 77,0.9112178\PG=C01 [X(C11H12O3)] \\@

P5-BASE.xyz-M062X-MG3S

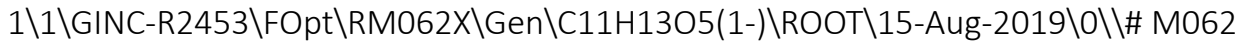
X/Gen SCF=Tight INT(grid=ultrafine) OPT IOP(2/17=4)\\title \-1,1\H,1.5 $389864945,1.0156883986,-1.7127411954 \backslash C, 0.8458915303,0.6013982096,-0.99$ 42826242\C,1.1559841827,-0.6778274176,-0.4763829507\C,2.3413814435,-1. $3431474709,-0.9358184385 \backslash \mathrm{C}, 0.269652096,-1.2431272204,0.4704500067 \backslash \mathrm{H}, 0$. $5132732659,-2.2013989886,0.9044519715 \backslash C,-0.8584991719,-0.5801073595,0$. $8682962423 \backslash 0,-1.7552982546,-1.0686444145,1.7873457488 \backslash C,-1.2275620659$, $0.7300412715,0.3560573567 \backslash \mathrm{C},-0.278752996,1.271958875,-0.6077306738 \backslash 0,-$ $0.6390815066,2.5089646269,-1.076467845 \backslash 0,-2.2509669095,1.3293423659,0$. $7065435008 \backslash \mathrm{C}, 0.2241941991,3.1041170353,-1.9976688081 \backslash \mathrm{C},-1.4659498182,-$ $2.3109256308,2.3492001392 \backslash \mathrm{H},-0.2086098543,4.0697631442,-2.2525431637 \backslash \mathrm{H}$ $, 0.3249440059,2.501510678,-2.9071527797 \backslash H, 1.2243002375,3.2551906919,-1$ $.5767005804 \backslash \mathrm{H},-2.2657699676,-2.5295855586,3.0545153731 \backslash \mathrm{H},-0.5075295088$ $,-2.3038012411,2.8808010759 \backslash \mathrm{H},-1.4303294758,-3.1023425232,1.5907436239$ 
$\backslash 0,3.1355038349,-0.8734938776,-1.751231337 \backslash C, 2.6277711317,-2.766718780$ $4,-0.4025025135 \backslash \mathrm{C}, 3.7463761746,-3.428901588,-1.1811034958 \backslash \mathrm{H}, 3.93434348$ $44,-4.4337367096,-0.7926934304 \backslash \mathrm{H}, 3.4842506705,-3.5052324597,-2.2350908$ $19 \backslash \mathrm{H}, 4.6520271671,-2.8308754327,-1.1042385418 \backslash \mathrm{H}, 1.7116409048,-3.360629$ $3008,-0.5041756731 \backslash 0,2.9645977383,-2.6571486356,0.9804178123 \backslash \mathrm{H}, 3.25889$ 09675,-3.5198306875,1.2809420189||Version=ES64L-G16RevB.01\State=1-A\H $\mathrm{F}=-803.0944951 \backslash \mathrm{RMSD}=6.307 \mathrm{e}-09 \backslash \mathrm{RMSF}=9.577 \mathrm{e}-06 \backslash \mathrm{Dipole}=1.4615734,-2.31393$ 34,-0.0768016\Quadrupole $=-16.7438771,10.0361642,6.7077129,11.4021281,9$ .794177,-6.1279479\PG=C01 [X(C11H13O5)]\\@

P5.xyz-M062X-MG3S

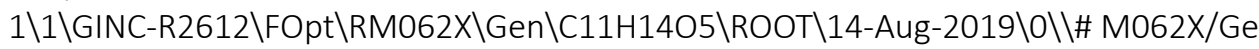

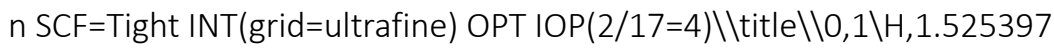
4783,0.964757112,-1.7535649938\C,0.8486731369,0.562411867,-1.015239575 $9 \backslash C, 1.1071455114,-0.704591881,-0.4959837938 \backslash C, 2.2947799062,-1.42801723$ $92,-1.0221967905 \backslash C, 0.2577590024,-1.2664068235,0.4545372262 \backslash H, 0.4848825$ $831,-2.2345226027,0.8698216888 \backslash \mathrm{C},-0.8578742259,-0.5650874975,0.8933885$ $575 \backslash 0,-1.742048603,-1.0120818507,1.8143688658 \backslash C,-1.1212985354,0.703376$ $986,0.3754436661 \backslash C,-0.2604997944,1.2569750585,-0.575909733 \backslash 0,-0.635497$ $1647,2.4993438253,-0.9931201008 \backslash 0,-2.2031584841,1.3817003986,0.8027696$ $171 \backslash \mathrm{H},-2.221499941,2.2287990124,0.3426651921 \backslash \mathrm{C}, 0.187071351,3.136979281$ $9,-1.9483490159 \backslash \mathrm{C},-1.4983651146,-2.276898482,2.3858322775 \backslash \mathrm{H},-0.2659075$ $906,4.1033736603,-2.145860308 \backslash \mathrm{H}, 0.2315692298,2.5564003647,-2.871434211$ 4\H,1.1969446365,3.2767406124,-1.5588357396\H,-2.2963485672,-2.4432823 $013,3.1027102311 \backslash \mathrm{H},-0.5345606451,-2.2991507817,2.8995841092 \backslash \mathrm{H},-1.52124$ $5814,-3.0635726812,1.6277488539 \backslash 0,2.9866509146,-0.9559404691,-1.893231$ $8376 \backslash C, 2.6158947857,-2.8242839645,-0.4685245439 \backslash C, 3.8420007311,-3.4095$ $156937,-1.1343932508 \backslash H, 4.0453573068,-4.4034676295,-0.7322934111 \backslash H, 3.69$ $16992917,-3.4957486447,-2.2081871671 \backslash H, 4.7030461496,-2.7658227134,-0.9$ $643068102 \backslash H, 1.7449554849,-3.4621542015,-0.671638239 \backslash 0,2.7862251796,-2$. $685498222,0.9360371856 \backslash H, 3.2917418005,-3.4355844998,1.2566220515 \backslash \backslash V e r s$ ion=ES64L-G16RevB.01 \State=1-A \HF=-803.6481812 \RMSD=5.309e-09\RMSF=1.0 33e-05 \Dipole $=0.1017418,-0.5687917,-0.0274253 \backslash$ Quadrupole $=-9.1565525,13$ $.5621845,-4.405632,-3.3283473,3.9602477,-8.2078965 \backslash P G=C 01[X(C 11 H 14 O 5)$ ]\@

PH1-BASE.xyz-M062X-MG3S

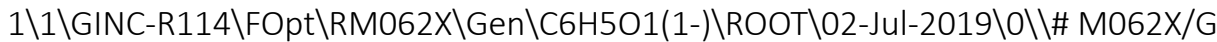

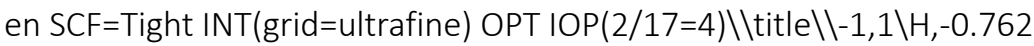
$9195879,3.9317548056,-0.0249718852 \backslash C,-0.3061082872,2.9881379783,0.2425$ $88635 \backslash C,-0.5883904911,1.8182324458,-0.4671498304 \backslash \mathrm{H},-1.2805502647,1.859$ $2683299,-1.3038325632 \backslash \mathrm{C},-0.0117187914,0.6082845351,-0.1352053172 \backslash \mathrm{H},-0$. $2452371955,-0.2874451818,-0.6999539746 \backslash C, 0.9156133484,0.464476476,0.95$ $81674216 \backslash 0,1.4475823841,-0.6343906773,1.2697488375 \backslash C, 1.1781362173,1.69$ $39383293,1.6621524329 \backslash \mathrm{H}, 1.870111353,1.6426523182,2.4954253614 \backslash \mathrm{C}, 0.5888$ $790802,2.8924029687,1.3111967517 \backslash H, 0.8293526864,3.7843971291,1.8833208$ 238||Version=ES64L-G16RevB.01 \State=1-A1 \HF=-306.8766291 \RMSD=9.719e-0 $9 \backslash \mathrm{RMSF}=1.917 \mathrm{e}-05 \backslash$ Dipole $=-0.7478366,1.5447762,-0.438018 \backslash$ Quadrupole $=1.70$ $11918,-5.4893405,3.7881486,4.9607295,-0.497909,3.3389686 \backslash P G=C 02 V[C 2(H$ 1C1C1O1),SGV(C4H4)]\\@

PH1.xyz-M062X-MG3S

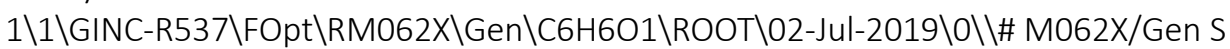

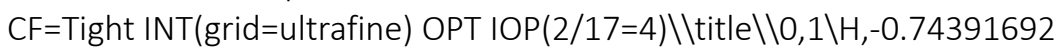


$85,3.9020293111,-0.010903119 \backslash C,-0.2885256068,2.9590098071,0.255107591 \backslash$ $\mathrm{C},-0.5835664483,1.8074398972,-0.4645760697 \backslash \mathrm{H},-1.2729625534,1.851485597$

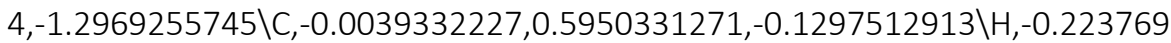
$413,-0.3089483259,-0.6802574552 \backslash C, 0.8827457276,0.5302840445,0.93827464$ $64 \backslash 0,1.4265766377,-0.6825393626,1.2290334101 \backslash \mathrm{H}, 2.0188636125,-0.6037222$ $965,1.9803643932 \backslash C, 1.184615208,1.6759954451,1.6645191132 \backslash H, 1.876909193$ ,1.6193552915,2.4965138085\C,0.5975673811,2.8848589516,1.3194948023\H, $0.8375108611,3.7722096383,1.88951212 \backslash \mid V e r s i o n=E S 64 L-G 16 R e v B .01 \backslash S t a t e=1$ $-A^{\prime} \backslash H F=-307.4438323 \backslash R M S D=6.479 e-09 \backslash R M S F=1.738 e-04 \backslash$ Dipole $=0.2460537,0.2$ $531375,0.3805668 \backslash$ Quadrupole $=-0.1868187,-1.0921651,1.2789838,-1.2107003$ ,4.6674074,-0.4907026\PG=CS [SG(C6H6O1)]\\@

PH2-BASE.xyz-M062X-MG3S

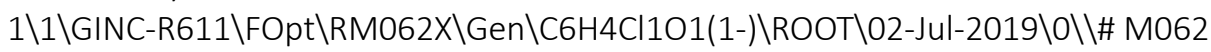
$\mathrm{X} /$ Gen SCF=Tight INT(grid=ultrafine) OPT IOP(2/17=4)\\title \\-1,1\H,0.6 $0473497,-2.4124058802,2.7154476038 \backslash C, 0.5957170527,-2.0867025446,1.6869$ $416909 \backslash \mathrm{C},-0.5687650601,-2.0607531655,0.9125309839 \backslash \mathrm{H},-1.5000478136,-2.3$ $822230357,1.3682363617 \backslash \mathrm{C},-0.5730232182,-1.6437030853,-0.4000925691 \backslash \mathrm{H},-$ 1.4913216931,-1.6347217073,-0.9747159976\C,0.6228626527,-1.2024471207, $-1.0746396882 \backslash 0,0.6400599995,-0.818915913,-2.2692030201 \backslash C, 1.8104047986$ $,-1.2391514384,-0.2527229417 \backslash \mathrm{H}, 2.7448002332,-0.9215193907,-0.694520350$ $1 \backslash C, 1.7570864227,-1.6643874045,1.0513556966 \backslash C l, 3.2573720468,-1.6795113$ 789,1.9915715726\|Version=ES64L-G16RevB.01\State=1-A'\HF=-766.4964267\ $\mathrm{RMSD}=4.710 \mathrm{e}-09 \backslash \mathrm{RMSF}=1.574 \mathrm{e}-04 \backslash \mathrm{Dipole}=0.2731388,-0.5746418,1.967661 \backslash$ Qua drupole $=4.0737959,5.5260948,-9.5998907,1.4292154,-5.9518362,4.0077316 \backslash$ $\mathrm{PG}=\mathrm{CS}[\mathrm{SG}(\mathrm{C} 6 \mathrm{H} 4 \mathrm{Cl} 1 \mathrm{O})]) \backslash @$

\section{PH2.xyz-M062X-MG3S}

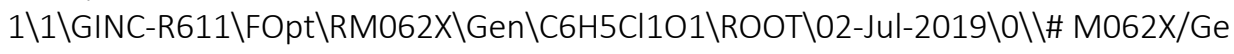
n SCF=Tight INT(grid=ultrafine) OPT IOP(2/17=4)\\title \} \backslash 0 , 1 \backslash \mathrm { H } , 0 . 6 0 5 0 4 4 $9166,-2.4018136409,2.6823370642 \backslash C, 0.599391786,-2.075389464,1.653576615$ $2 \backslash \mathrm{C},-0.5691058684,-2.0575866774,0.9023722356 \backslash \mathrm{H},-1.4962955313,-2.378041$ $3994,1.3573199206 \backslash \mathrm{C},-0.569008538,-1.6378286925,-0.4161561893 \backslash \mathrm{H},-1.4738$ $011015,-1.6213467976,-1.0063012242 \backslash C, 0.6231181182,-1.225960662,-1.0006$ $175934 \backslash 0,0.5801575402,-0.821843829,-2.2956327994 \backslash \mathrm{H}, 1.4556415632,-0.561$ $6926106,-2.591914939 \backslash \mathrm{C}, 1.8045392482,-1.2351609467,-0.2687465277 \backslash \mathrm{H}, 2.73$ $78121124,-0.9164413283,-0.7146240977 \backslash C, 1.7733360552,-1.6606559539,1.04$ $9294977 \backslash \mathrm{Cl}, 3.2518178545,-1.6696882589,1.9573956583 \backslash \mid V e r s i o n=E S 64 L-G 16 R$ evB.01\State $=1-A^{\prime} \backslash H F=-767.0505772 \backslash R M S D=9.971 e-09 \backslash R M S F=1.728 e-04 \backslash$ Dipole $=-0.0778504,0.0896136,-0.3278258 \backslash$ Quadrupole $=1.1463168,-2.0854225,0.939$ 1057,2.3450591,-4.7566193,-2.2313491\PG=CS [SG(C6H5Cl101)]\\@

PH3-BASE.xyz-M062X-MG3S

$1 \backslash 1 \backslash G I N C-R 949 \backslash F O p t \backslash R M 062 X \backslash G e n \backslash C 6 H 4 N 1 O 3(1-) \backslash R O O T \backslash 02-J u l-2019 \backslash 0 \backslash \backslash \#$ M062X

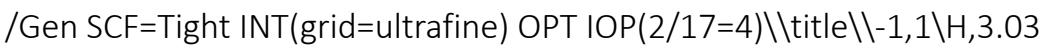
20217686,-1.2507073519,-2.8500013542\C,2.2851293463,-0.5561411623,-2.5 $057012223 \backslash \mathrm{C}, 0.9880197242,-0.9753984297,-2.2232533938 \backslash \mathrm{N}, 0.6765919172,-2$ $.405962161,-2.4135584186 \backslash \mathrm{C},-0.0179906649,-0.1462010798,-1.7801439668 \backslash \mathrm{H}$ ,-1.0027088719,-0.5400629266,-1.580388546\С,0.2175405297,1.2614463283, $-1.5729988139 \backslash 0,-0.6714137897,2.0473952715,-1.1711159801 \backslash C, 1.566220801$ $6,1.6746427368,-1.8710878233 \backslash \mathrm{H}, 1.7945558881,2.7245903616,-1.7313497151$ \C,2.5381762068,0.8006334208,-2.3133428013\Н,3.5338238049,1.1787047058 $,-2.5192285297 \backslash 0,-0.4486910263,-2.7974886211,-2.1739233072 \backslash 0,1.5637241$ $182,-3.1437209214,-2.8055770087 \backslash \mid$ Version=ES64L-G16RevB.01\State=1-A'\H 
$\mathrm{F}=-511.4054534 \backslash \mathrm{RMSD}=8.834 \mathrm{e}-09 \backslash \mathrm{RMSF}=2.078 \mathrm{e}-04 \backslash \mathrm{Dipole}=1.4130724,-0.87222$

$5,-0.5656357 \backslash$ Quadrupole $=4.1884924,-12.5792317,8.3907393,6.9272607,3.11$

82543,-6.424811\PG=CS [SG(C6H4N1O3)]\\@

PH3.xyz-M062X-MG3S

$1 \backslash 1 \backslash G I N C-R 965 \backslash F O p t \backslash R M 062 X \backslash G e n \backslash C 6 H 5 N 103 \backslash R O O T \backslash 02-J u l-2019 \backslash 0 \backslash \backslash \#$ M062X/Gen SCF=Tight INT(grid=ultrafine) OPT IOP(2/17=4)\\title $\backslash 0,1 \backslash H, 3.0107047$

$948,-1.2352178293,-2.8410637622 \backslash C, 2.2644362686,-0.5375159633,-2.496300$

$0781 \backslash \mathrm{C}, 0.9843850059,-0.9735744233,-2.2218653977 \backslash \mathrm{N}, 0.6633083699,-2.4037$ $500335,-2.4093959485 \backslash \mathrm{C},-0.0236817756,-0.1372582969,-1.7767582884 \backslash \mathrm{H},-1$. $0074184469,-0.5392903778,-1.5788398011 \backslash C, 0.2724490842,1.2057956724,-1$. $5991404 \backslash 0,-0.6464582195,2.1033290884,-1.1671615823 \backslash \mathrm{H},-1.4915906695,1.6$ $734390877,-1.0135309019 \backslash \mathrm{C}, 1.5543975426,1.6801314262,-1.8666776414 \backslash \mathrm{H}, 1$. $7570622229,2.7315406914,-1.7194493781 \backslash C, 2.536089072,0.8119031052,-2.31$ $05802578 \backslash \mathrm{H}, 3.5278087857,1.1894267749,-2.5154951972 \backslash 0,-0.4671045889,-2$. 7596260302,-2.16137364\0,1.5494106766,-3.1283415931,-2.7986044166||Ver sion=ES64L-G16RevB.01\State $=1-A^{\prime} \backslash H F=-511.9482684 \backslash$ RMSD $=4.677 \mathrm{e}-09 \backslash \mathrm{RMSF}=8$ $.190 \mathrm{e}-05 \backslash$ Dipole $=0.0515529,1.4060103,0.2584455 \backslash$ Quadrupole $=7.1491045,-7$. 4004834,0.2513789,-0.3386213,-2.093138,-1.3262384\PG=CS [SG(C6H5N1O3)] 11@

PH4-BASE.xyz-M062X-MG3S

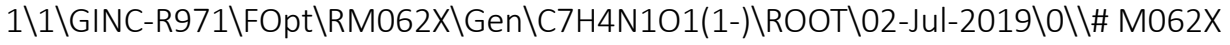

/Gen SCF=Tight INT(grid=ultrafine) OPT IOP(2/17=4)\title \-1,1\C,1.34 $15714456,-1.2803937075,2.1420545964 \backslash C, 0.2697357182,-0.778697059,1.3625$ $389017 \backslash \mathrm{C},-1.0682793996,-0.9402149305,1.7755792245 \backslash \mathrm{H},-1.2637588221,-1.4$ $605192601,2.7066149467 \backslash \mathrm{C},-2.1093575299,-0.4570384882,1.0301136036 \backslash \mathrm{H},-3$ $.1323636921,-0.5883224119,1.3600355774 \backslash \mathrm{C},-1.9104977988,0.2452883335,-0$ $.2200603929 \backslash 0,-2.8546285032,0.6897646128,-0.9050463004 \backslash C,-0.523025089$, $0.3891646046,-0.6075392468 \backslash \mathrm{H},-0.326232237,0.9087769393,-1.5370068574 \backslash \mathrm{C}$ $, 0.5064321167,-0.1006316306,0.1496646182 \backslash H, 1.5313751897,0.0303614636,-$ $0.1791196698 \backslash \mathrm{N}, 2.2155586014,-1.688718466,2.7783209988 \mid \backslash V e r s i o n=E S 64 L-G$

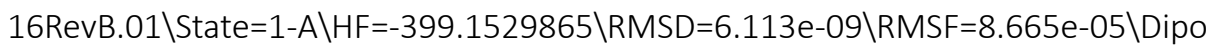
$l e=0.3158371,-0.1504291,0.2277792 \backslash$ Quadrupole $=-8.7023847,7.1084332,1.59$ 39515,10.9097929,-17.1952064,6.5626101\PG=C01 [X(C7H4N1O1)]\\@

PH4.xyz-M062X-MG3S

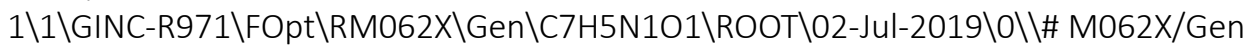

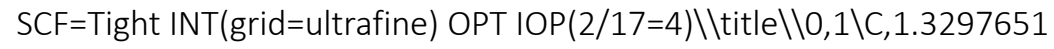

$1,-1.302018686,2.1190096246 \backslash C, 0.2493777452,-0.7686125233,1.3431526393 \backslash$

C,-1.0700180814,-0.9343858152,1.7685472256\Н,-1.2700491731,-1.46775206 $96,2.6870530783 \backslash \mathrm{C},-2.1109518846,-0.4215171255,1.0226019085 \backslash \mathrm{H},-3.138449$ $257,-0.539423281,1.3350650529 \backslash C,-1.8417309292,0.2638317315,-0.15893105$ $44 \backslash 0,-2.9006720483,0.7448292669,-0.8492824014 \backslash \mathrm{H},-2.6068656507,1.197928$ $4704,-1.6434997914 \backslash \mathrm{C},-0.5292339143,0.4330092731,-0.5891362827 \backslash \mathrm{H},-0.325$ $7009322,0.9669967519,-1.5089412155 \backslash C, 0.5122743031,-0.0819548475,0.1601$ $955 \backslash \mathrm{H}, 1.5339940342,0.0458649658,-0.1681396399 \backslash \mathrm{N}, 2.1951006782,-1.729066$ $1116,2.7405353562 \backslash \mid$ Version=ES64L-G16RevB.01\State=1-A\HF=-399.6901286\ RMSD =9.202e-09\RMSF=5.324e-05\Dipole $=-1.1271953,0.912531,-1.4079987 \backslash Q u$ adrupole $=-5.2229821,1.0328698,4.1901123,4.3464427,-6.7042374,-1.956449$ $5 \backslash P G=C 01[X(C 7 H 5 N 101)] \backslash \backslash @$

PH5-BASE.xyz-M062X-MG3S

$1 \backslash 1 \backslash G I N C-R 971 \backslash F O p t \backslash R M 062 X \backslash G e n \backslash C 6 H 4 N 1 O 3(1-) \backslash R O O T \backslash 02-J u l-2019 \backslash 0 \backslash \backslash \#$ M062X 
/Gen SCF=Tight INT(grid=ultrafine) OPT IOP(2/17=4)\\itle\\-1,1\N,1.50 $3253336,-1.2916031294,-2.6669213451 \backslash C, 0.6973478745,-0.4762044652,-1.84$ $3665137 \backslash \mathrm{C},-0.7011461048,-0.6326044534,-1.8436988272 \backslash \mathrm{H},-1.1322575355,-1$ $.3875297555,-2.4862290111 \backslash \mathrm{C},-1.48489119,0.1521299081,-1.0505742637 \backslash \mathrm{H},-$ $2.5612297823,0.0363589491,-1.0464171886 \backslash C,-0.9332305227,1.1735814099,-$ $0.1779811921 \backslash 0,-1.6429552097,1.8916663336,0.5470235169 \backslash C, 0.5138354096$, $1.2890801768,-0.2200710187 \backslash \mathrm{H}, 0.9619501661,2.0404751649,0.417521091 \backslash \mathrm{C}, 1$ $.2871505324,0.4984128635,-1.0175294005 \backslash \mathrm{H}, 2.3631912263,0.6008119265,-1$. $0338134884 \backslash 0,2.7265192937,-1.1383348789,-2.6519225996 \backslash 0,0.9665181666$,2.1394891912,-3.3832315477\\Version=ES64L-G16RevB.01 \State=1-A1 \HF=-51 1.420773 \RMSD $=4.686 \mathrm{e}-09 \backslash \mathrm{RMSF}=4.140 \mathrm{e}-05 \backslash \mathrm{Dipole}=0.3113739,-0.3150418,-0$. $3180776 \backslash$ Quadrupole $=0.1271637,0.0341806,-0.1613443,12.0030299,12.163755$ 8,-12.493395\PG=C02V [C2(N1C1C1O1),SGV(C4H4O2)]\\@

PH5.xyz-M062X-MG3S

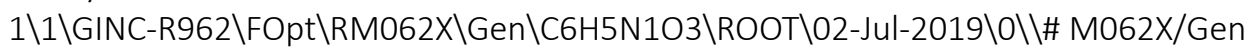

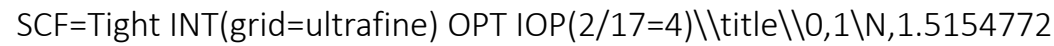
959,-1.2973695979,-2.6734519994\C,0.6764122377,-0.4507084394,-1.818397 $1444 \backslash \mathrm{C},-0.6985973635,-0.6256377426,-1.8377003272 \backslash \mathrm{H},-1.1296548226,-1.37$ $92744452,-2.479095532 \backslash \mathrm{C},-1.4867988548,0.1710887936,-1.0332150793 \backslash \mathrm{H},-2$. $5618498245,0.0663626042,-1.0191745844 \backslash \mathrm{C},-0.893487334,1.133484173,-0.21$

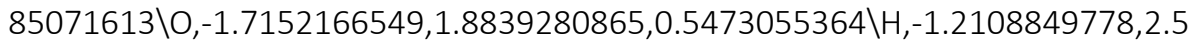
$137901342,1.0687184861 \backslash \mathrm{C}, 0.4899532368,1.2965677798,-0.2108308749 \backslash \mathrm{H}, 0.9$ 42708447, 2.0467553489,0.4252232553\C,1.2818099274,0.4995041268,-1.0159 $941049 \backslash \mathrm{H}, 2.3560467863,0.6037478609,-1.0303905085 \backslash 0,2.7137576399,-1.121$ 346107,-2.6351930415\0,0.9592741942,-2.1211219769,-3.3658775784\\Versi on $=E S 64 L-G 16 R e v B .01 \backslash$ State $=1-A^{\prime} \backslash H F=-511.950369 \backslash R M S D=4.308 e-09 \backslash R M S F=6.62$ 7e-05\Dipole $=-0.7135463,1.3969304,1.342601 \backslash$ Quadrupole $=-2.2964265,1.738$ $3368,0.5580897,4.4374464,4.2460049,1.3253313 \backslash P G=C S[S G(C 6 H 5 N 103)] \backslash \backslash @$

PY1-BASE.xyz-M062X-MG3S

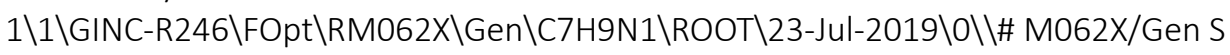

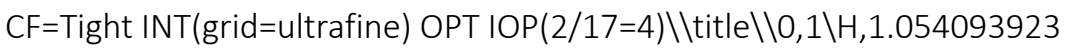
4,0.8332220734,-2.6482753347\C,0.5773239009,0.13558346886,-1.9723156328 \C,-0.7975749086,-0.0225287197,-1.9801455851 \H,-1.4224111581,0.5438626 $368,-2.6575607789 \backslash \mathrm{C},-1.3740872837,-0.9298979735,-1.095431977 \backslash \mathrm{C},-2.8627$ $203803,-1.1363094846,-1.0667246279 \backslash N,-0.6424208811,-1.649220541,-0.242$ $9738534 \backslash C, 0.6830794993,-1.4996094755,-0.2324475201 \backslash C, 1.4606755449,-2.3$ $336301912,0.7469462494 \backslash \mathrm{C}, 1.332895667,-0.6125409644,-1.0864100807 \backslash \mathrm{H}, 2.4$ $098055492,-0.5174309609,-1.0499402977 \backslash \mathrm{H},-3.1161976026,-1.8785283038,-0$ $.3153146404 \backslash \mathrm{H},-3.3783218684,-0.2043111916,-0.8329576193 \backslash \mathrm{H},-3.225218240$ $2,-1.476008593,-2.0374524706 \backslash \mathrm{H}, 0.7804477571,-2.9576647641,1.3193337542$ $\backslash \mathrm{H}, 2.1768967597,-2.9720696202,0.2287426272 \backslash H, 2.0237931315,-1.700372218$

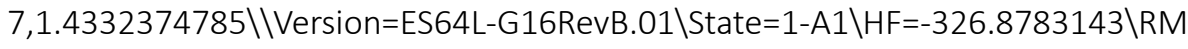
$\mathrm{SD}=4.220 \mathrm{e}-09 \backslash \mathrm{RMSF}=5.636 \mathrm{e}-05 \backslash \mathrm{Dipole}=0.2718748,0.3978236,-0.3854614 \backslash \mathrm{Quad}$ rupole $=2.7114791,-1.5847651,-1.126714,-0.7564111,1.5250096,-1.5937351 \backslash$ PG=C02V [C2(H1C1N1),SGV(C6H4),X(H4)]\\@

PY1.xyz-M062X-MG3S

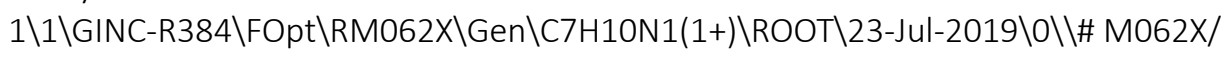

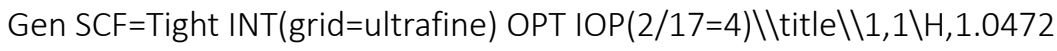
$004656,0.8224658204,-2.6376391881 \backslash C, 0.5705407706,0.1251789271,-1.96204$ $79876 \backslash \mathrm{C},-0.8089293229,-0.020013394,-1.9844069482 \backslash \mathrm{H},-1.4240184619,0.550$ $27462,-2.6649274448 \backslash C,-1.4137144085,-0.9107044245,-1.1209091664 \backslash C,-2.8$ 
859342577,-1.1467022514,-1.0591945039\N,-0.6129949956,-1.6061694252,-0 $.2845695736 \backslash C, 0.732326269,-1.5049575992,-0.2201186525 \backslash C, 1.4646020172,-$ $2.3513951635,0.7669227027 \backslash \mathrm{C}, 1.3439795081,-0.6161684068,-1.080733559 \backslash \mathrm{H}$, $2.418860665,-0.5138445951,-1.0518969462 \backslash \mathrm{H},-1.0601480169,-2.2602921554$, $0.3492004926 \backslash \mathrm{H},-3.1499165508,-1.889002161,-0.3089881337 \backslash \mathrm{H},-3.400456295$ 4,-0.2160626807,-0.8228477929\Н,-3.24694103,-1.4889713242,-2.028322306 $6 \backslash \mathrm{H}, 0.7912196867,-2.9803294344,1.3452853263 \backslash \mathrm{H}, 2.1779613087,-2.99116346$ $39,0.2487550187 \backslash \mathrm{H}, 2.0244460433,-1.7182548204,1.4542295324 \backslash \backslash V e r s i o n=E S 6$

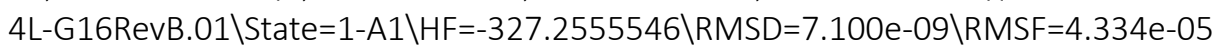
$\backslash$ Dipole $=-0.1809002,-0.2646319,0.256398 \backslash$ Quadrupole $=5.8076952,-3.3892546$ $,-2.4184406,0.0124618,1.9586884,-6.6370314 \backslash P G=C 02 V$ [C2(H1C1N1H1),SGV(C 6H4),X(H4)]\\@

PY2-BASE.xyz-M062X-MG3S

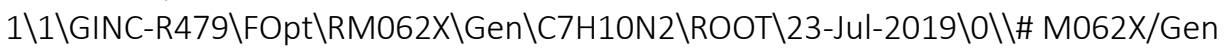
SCF=Tight INT(grid=ultrafine) OPT IOP(2/17=4)\|title \\0,1\N,1.22279917

$88,-1.8603971976,-1.7832100323 \backslash C, 0.5628643305,-1.1210364436,-0.8444898$ $441 \backslash \mathrm{C},-0.8363545384,-0.9850273342,-0.8531571053 \backslash \mathrm{H},-1.4454426819,-1.461$ $6837084,-1.6051608186 \backslash \mathrm{C},-1.4466699289,-0.222443235,0.1250321755 \backslash \mathrm{H},-2.5$ 262364651,-0.119452602,0.1156261676\N,-0.8072301973,0.4139546633,1.104 $3925287 \backslash C, 0.5177875253,0.2822624059,1.1085567661 \backslash H, 1.0450286846,0.7980$ $718078,1.9036145441 \backslash \mathrm{C}, 1.2425533194,-0.4509172569,0.1876681848 \backslash \mathrm{H}, 2.3165$ 752328,-0.4951513671,0.2783297713\C,0.4767276964,-2.5306146038,-2.8249 $262744 \backslash \mathrm{C}, 2.6637769842,-1.9687209827,-1.7299589332 \backslash \mathrm{H},-0.2301698877,-3.2$ $53834835,-2.4096367598 \backslash \mathrm{H}, 1.1668767979,-3.0638042903,-3.4714791539 \backslash \mathrm{H},-0$ $.0843743598,-1.8194017278,-3.4369375533 \backslash \mathrm{H}, 2.9970626366,-2.4246988025,-$ $0.7938916387 \backslash H, 3.1428581645,-0.9902656953,-1.8211924323 \backslash H, 3.0053796828$

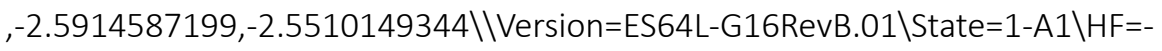
382.2120038\RMSD=6.059e-09\RMSF=4.021e-05\Dipole $=0.8974209,-1.0054292$, $-1.2765307 \backslash$ Quadrupole=3.1387234,-2.0568106,-1.0819127,1.4962238,2.8671 231,0.6115162\PG=C02V [C2(N1C1N1),SGV(C6H6),X(H4)]\\@

PY2.xyz-M062X-MG3S

$1 \backslash 1 \backslash G I N C-R 480 \backslash F O p t \backslash R M 062 X \backslash G e n \backslash C 7 H 11 N 2(1+) \backslash R O O T \backslash 23-J u l-2019 \backslash 0 \backslash \backslash \#$ M062X/

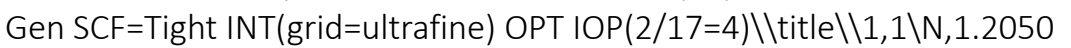
417088,-1.8403718932,-1.7579834161\C,0.5631970973,-1.1213653222,-0.845 $0062472 \backslash \mathrm{C},-0.8574990463,-0.9865080419,-0.8582772704 \backslash \mathrm{H},-1.460550172,-1$. 4647960309,-1.6117506025\C,-1.4811713602,-0.2435594099,0.090712237\\,$2.5529792291,-0.1163425256,0.1162778251 \backslash \mathrm{N},-0.77717638,0.3801466022,1.0$ $615773098 \backslash C, 0.5699304308,0.2834264803,1.1176626871 \backslash H, 1.0570592891,0.81$ $1178171,1.923760368 \backslash \mathrm{C}, 1.2579372083,-0.4429928139,0.2008843096 \backslash \mathrm{H}, 2.3305$ $781263,-0.4907482632,0.2864003957 \backslash \mathrm{H},-1.265430432,0.9270981001,1.756083$ $1046 \backslash C, 0.4635294836,-2.5232985918,-2.8168704418 \backslash C, 2.6616717871,-1.9585$ $338476,-1.7162994035 \backslash \mathrm{H},-0.2325194149,-3.248385576,-2.3947839004 \backslash \mathrm{H}, 1.16$ $5093193,-3.0499369362,-3.4527421716 \backslash \mathrm{H},-0.0860079425,-1.806523241,-3.42$ $73079618 \backslash \mathrm{H}, 2.9848611484,-2.4217497808,-0.7838982624 \backslash \mathrm{H}, 3.1313726208,-0$. $9798874458,-1.8164223237 \backslash \mathrm{H}, 2.9867083011,-2.5819126463,-2.5406916515 \backslash \backslash \mathrm{V}$ ersion=ES64L-G16RevB.01 \State=1-A1 \HF=-382.6048615\RMSD=7.523e-09\RMSF $=8.367 \mathrm{e}-05 \backslash$ Dipole $=-0.4414597,0.4945316,0.6279443 \backslash$ Quadrupole $=2.4855111$, $-4.6892637,2.2037526,-5.0471173,-4.7503875,11.8763153$ \PG=C02V [C2(N1C1 $\mathrm{N} 1 \mathrm{H} 1), \mathrm{SGV}(\mathrm{C} 6 \mathrm{H} 6), \mathrm{X}(\mathrm{H} 4)] \backslash \backslash @$

PY3-BASE.xyz-M062X-MG3S

$1 \backslash 1 \backslash G I N C-R 523 \backslash F O p t \backslash R M 062 X \backslash G e n \backslash C 8 H 11 N 1 \backslash R O O T \backslash 23-J u l-2019 \backslash 0 \backslash \backslash \#$ M062X/Gen 
SCF=Tight INT(grid=ultrafine) OPT IOP(2/17=4)\\title \\0,1\C,0.95880325 09,-1.876194883,2.2738257229\C,0.0297831047,-0.8372289012,1.7123904112 $\backslash \mathrm{C},-1.3486277639,-0.9830303314,1.8142807688 \backslash \mathrm{H},-1.7734233696,-1.8522607$ $263,2.302400095 \backslash C,-2.1866730194,-0.0086360871,1.287229752 \backslash C,-3.6807491$ $622,-0.1457680193,1.3860876519 \backslash \mathrm{N},-1.7144756631,1.0809662541,0.67628302$ $58 \backslash C,-0.3954078167,1.2352664276,0.5705924199 \backslash C, 0.0984152589,2.47408130$ $88,-0.1233597001 \backslash C, 0.5038366149,0.2993763366,1.0753723465 \backslash \mathrm{H}, 1.56891707$ $31,0.4642914809,0.9676893283 \backslash H, 2.0001108851,-1.607482075,2.1089954909 \backslash$ $\mathrm{H}, 0.776830743,-2.845423261,1.8090583191 \backslash \mathrm{H}, 0.8003644179,-1.9938428465,3$ $.3460115626 \backslash \mathrm{H},-4.1595827324,0.7101144727,0.9192002845 \backslash \mathrm{H},-3.9964936539$, $-0.2036522623,2.4281988017 \backslash \mathrm{H},-4.0200420869,-1.0557667079,0.8902817258 \backslash$ $\mathrm{H},-0.7466092458,3.0764082779,-0.4441523593 \backslash \mathrm{H}, 0.7011765685,2.2157283451$ $,-0.9946238326 \backslash \mathrm{H}, 0.7247244371,3.0678223688,0.5432563856 \backslash \backslash$ Version=ES64L

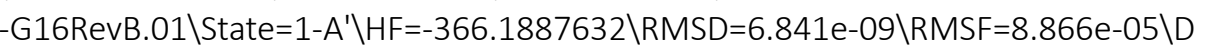
ipole $=0.4937809,-0.5601285,0.30279 \backslash$ Quadrupole $=1.6500306,0.0882123,-1.7$ 382429,1.7659571,-1.0434185,-1.5120756\PG=CS [SG(C8H5N1),X(H6)]\\@

PY3.xyz-M062X-MG3S

$1 \backslash 1 \backslash G I N C-R 649 \backslash F O p t \backslash R M 062 X \backslash G e n \backslash C 8 H 12 N 1(1+) \backslash R O O T \backslash 23-J u l-2019 \backslash 0 \backslash \backslash \#$ M062X/ Gen SCF=Tight INT(grid=ultrafine) OPT IOP(2/17=4)\title \1,1\C,0.9521 $94432,-1.8647037557,2.2682445777 \backslash \mathrm{C}, 0.0294847451,-0.8306456717,1.709139$ $4631 \backslash \mathrm{C},-1.3540631017,-0.9900080908,1.8185412671 \backslash \mathrm{H},-1.7694187142,-1.860$ $8554841,2.3075598139 \backslash C,-2.2137283849,-0.0462440667,1.3085241889 \backslash C,-3.7$ $02087345,-0.1432268052,1.3849464061 \backslash \mathrm{N},-1.6705352848,1.0335677809,0.701$ $7340262 \backslash C,-0.3463902759,1.25305791,0.5599093207 \backslash C, 0.0980331235,2.49677$ $0876,-0.1362237005 \backslash C, 0.5167098802,0.3081800189,1.070491482 \backslash H, 1.5808267$ $768,0.4677880727,0.9658182364 \backslash \mathrm{H},-2.3048434209,1.7292625931,0.325784628$ $6 \backslash \mathrm{H}, 1.994455501,-1.6048864044,2.1083602085 \backslash \mathrm{H}, 0.7544986646,-2.831050241$ $5,1.8033305534 \backslash \mathrm{H}, 0.7778774309,-1.9801656583,3.3384484504 \backslash \mathrm{H},-4.19072197$ $82,0.7100688522,0.9194235649 \backslash \mathrm{H},-4.0178189802,-0.1970817685,2.425913343$ $8 \backslash \mathrm{H},-4.0412619712,-1.0503038475,0.8865782689 \backslash \mathrm{H},-0.7431751518,3.1054745$ $346,-0.4608047299 \backslash H, 0.696899511,2.2394750882,-1.0089001814 \backslash H, 0.7203392$ 9,3.0925802656,0.5302239861 \|Version=ES64L-G16RevB.01\State=1-A' $\backslash H F=-3$ 66.5719205\RMSD=9.095e-09\RMSF=5.594e-05\Dipole $=-0.4514043,0.4766819,-$ $0.2573405 \backslash$ Quadrupole $=4.9884611,1.8852513,-6.8737125,-0.7097397,0.15376$ 89,-6.9904583\PG=CS [SG(C8H6N1),X(H6)]\\@

PY4-BASE.xyz-M062X-MG3S

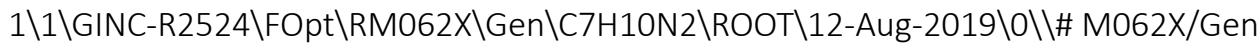

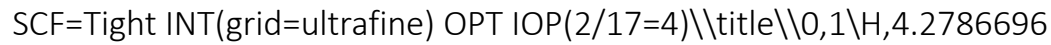
421,0.1954668196,1.2868136341\C,3.3658151245,-0.3714028994,1.183452375 $9 \backslash C, 2.1856041361,0.0421293297,1.8145750154 \backslash N, 2.1194509746,1.1993267244$ ,2.564894193\C,1.0615843046,-0.7880183413,1.6368173277\Н,0.1216096511, $-0.5451786283,2.1135037609 \backslash N, 1.0615839373,-1.8895541504,0.9014205064 \backslash \mathrm{C}$ ,2.1872590699,-2.2532996934,0.2945109687 $\backslash \mathrm{H}, 2.1506701112,-3.1581369648$, $-0.298612859 \backslash \mathrm{C}, 3.358273116,-1.5222946021,0.4166930809 \backslash \mathrm{H}, 4.2607713373,-$ $1.8518439074,-0.0792518659 \backslash C, 0.9589058154,1.4175141087,3.4025908957 \backslash C$, $3.3572680292,1.8507473039,2.9334350415 \backslash \mathrm{H}, 0.8168671362,0.6179111236,4.1$ $391288897 \backslash \mathrm{H}, 0.0538508837,1.4897859692,2.7992476749 \backslash \mathrm{H}, 1.0783216609,2.35$ $85815912,3.9316748695 \backslash H, 3.8980342841,2.1843809078,2.0470750771 \backslash H, 4.019$ $6759019,1.1960870657,3.5127963751 \backslash H, 3.130784884,2.7294082434,3.5304250$ $381 \backslash \backslash$ Version=ES64L-G16RevB.01 \State $=1-A \backslash H F=-382.2060463 \backslash R M S D=5.124 \mathrm{e}-09$ $\backslash$ RMSF $=1.138$ e-05 \Dipole $=0.7116876,1.0531955,0.6444089 \backslash$ Quadrupole $=0.4879$ $893,-0.6618341,0.1738448,-2.6131312,-2.9807384,2.9394407 \backslash P G=C 01[X(C 7 H$ 
10N2)]入\@

PY4.xyz-M062X-MG3S

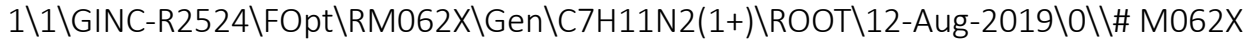

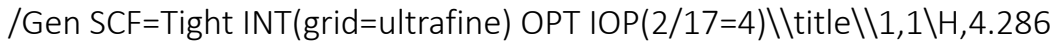

$0768496,0.1267940975,1.358969958 \backslash C, 3.3681107906,-0.4256402364,1.227561$

$8135 \backslash C, 2.1944807391,-0.0063402213,1.9033194754 \backslash N, 2.1657513652,1.064705$

$9127,2.7153677976 \backslash \mathrm{C}, 1.0459331373,-0.782481905,1.674924835 \backslash \mathrm{H}, 0.08708553$

$44,-0.5805876039,2.1214368711 \backslash \mathrm{N}, 1.1062816794,-1.8443303434,0.860199749$

$8 \backslash C, 2.2024850759,-2.2536719279,0.2158757817 \backslash H, 2.1145310801,-3.12593271$

$44,-0.4109120508 \backslash C, 3.3644673303,-1.527585627,0.4025283976 \backslash H, 4.26606145$

$44,-1.8348021274,-0.1054275746 \backslash H, 0.2515832787,-2.3727723746,0.72706970$

$32 \backslash C, 0.9243732135,1.4372289981,3.376193029 \backslash C, 3.3761466636,1.8466669037$

,2.929765781 \H,0.5716779483,0.6374103969,4.0311619199\Н,0.1460909417,1

$.6737868775,2.6472369149 \backslash \mathrm{H}, 1.0974661178,2.3185198831,3.9828799788 \backslash \mathrm{H}, 3$.

$7394588897,2.274707332,1.9939462145 \backslash H, 4.1645757702,1.2377353141,3.3753$ $37345 \backslash H, 3.1498321402,2.6598693656,3.6097940594 \backslash \backslash V e r s i o n=E S 64 L-G 16 R e v B$. $01 \backslash S t a t e=1-A \backslash H F=-382.5867988 \backslash R M S D=7.233 e-09 \backslash R M S F=7.048 \mathrm{e}-06 \backslash$ Dipole $=-0.6$ $048058,-0.837164,-0.4415787 \backslash$ Quadrupole $=0.614685,3.6624026,-4.2770876,4$ $.1358001,-1.1113002,11.2750398 \backslash P G=C 01[X(C 7 H 11 N 2)] \backslash \backslash @$

PY5-BASE.xyz-M062X-MG3S

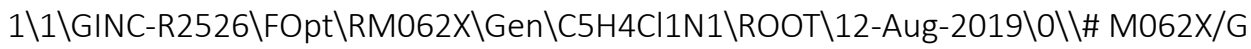

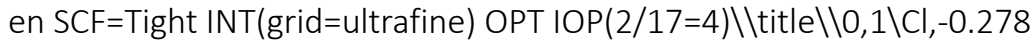
4801197,-2.1548751301,-2.2144926256\C,-0.7406780679,-0.6766347611,-1.4 $459850208 \backslash \mathrm{C},-1.891625157,-0.0195841057,-1.842775652 \backslash \mathrm{H},-2.5157743897,-0$ $.4134418171,-2.6311217632 \backslash \mathrm{C},-2.2123287973,1.1623180563,-1.1901884563 \backslash \mathrm{H}$ $,-3.1056198875,1.7067182676,-1.4718380423 \backslash \mathrm{N},-1.4814678787,1.6926215449$ $,-0.2142560497 \backslash \mathrm{C},-0.3807388601,1.0399817536,0.1466878033 \backslash \mathrm{H}, 0.202964858$ $2,1.4857299249,0.9430957316 \backslash C, 0.039726049,-0.1485837037,-0.4330837097 \backslash$ H,0.942046951,-0.6443980314,-0.1072602993\|Version=ES64L-G16RevB.01\St

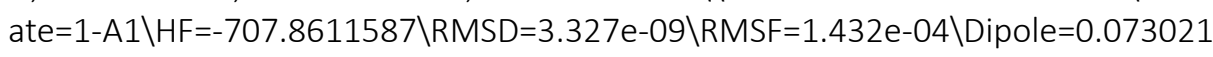
$8,-0.2335444,-0.1214151 \backslash$ Quadrupole $=3.128446,-3.6684783,0.5400323,0.438$ 7656,3.7870347,-1.4733753\PG=C02V [C2(N1C1Cl1),SGV(C4H4)]\\@

PY5.xyz-M062X-MG3S

$1 \backslash 1 \backslash G I N C-R 2920 \backslash F O p t \backslash R M 062 X \backslash G e n \backslash C 5 H 5 C l 1 N 1(1+) \backslash R O O T \backslash 12-A u g-2019 \backslash 0 \backslash \backslash \#$ M06 2X/Gen SCF=Tight INT(grid=ultrafine) OPT IOP(2/17=4)\\title $\backslash 1,1 \backslash \mathrm{Cl},-0$ $.29012514,-2.1174203252,-2.1949919643 \backslash \mathrm{C},-0.7426534957,-0.6701533014,-1$ $.4425704207 \backslash \mathrm{C},-1.9047403502,-0.0097257951,-1.8454935656 \backslash \mathrm{H},-2.525659864$ 2,-0.4071125867,-2.6340417691\C,-2.2471837477,1.1602766497,-1.21895246 $53 \backslash \mathrm{H},-3.1283427153,1.7315699135,-1.4705880421 \backslash \mathrm{N},-1.4671962674,1.647064$ $72,-0.2378689165 \backslash C,-0.34302081,1.0330777713,0.1709312697 \backslash H, 0.210850133$ $7,1.5085104384,0.966750558 \backslash C, 0.0472014715,-0.1401163229,-0.4207351397 \backslash$ $\mathrm{H}, 0.9484220461,-0.6391827074,-0.098245149 \backslash \mathrm{H},-1.7382107831,2.5138178563$ , $0.2127485038 \backslash \mid$ Version=ES64L-G16RevB.01\State=1-A1\HF=-708.2194601\RMS $\mathrm{D}=3.256 \mathrm{e}-09 \backslash \mathrm{RMSF}=4.466 \mathrm{e}-05 \backslash \mathrm{Dipole}=-0.6351736,2.0313995,1.0561069 \backslash \mathrm{Quadr}$ upole $=-1.5838517,4.1417329,-2.5578813,-5.0615684,3.0824143,7.2215715 \backslash P$ $\mathrm{G}=\mathrm{C} 02 \mathrm{~V}[\mathrm{C} 2(\mathrm{H} 1 \mathrm{~N} 1 \mathrm{C} 1 \mathrm{Cl} 1), \mathrm{SGV}(\mathrm{C} 4 \mathrm{H} 4)] \backslash \backslash @$

PYR1-BASE.xyz-M062X-MG3S

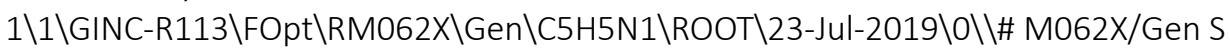
$\mathrm{CF}=$ Tight INT(grid=ultrafine) OPT IOP(2/17=4)\|title \} \backslash 0 , 1 \backslash H , 1 . 8 9 9 6 9 9 3 7 7 4,0.0698709139,2.4094652392\C,1.2079374446,0.3747477343,1.6358336156\C 
$,-0.159541838,0.2394688287,1.8165314744 \backslash \mathrm{H},-0.5669288168,-0.171550893,2$ $.7293995199 \backslash \mathrm{C},-1.0043203274,0.6457813093,0.7929943834 \backslash \mathrm{H},-2.078817232,0$ $.5544421563,0.9003208263 \backslash \mathrm{N},-0.572678412,1.1595097673,-0.3555172683 \backslash \mathrm{C}, 0$ $.7417718163,1.2841624772,-0.5167406694 \backslash \mathrm{H}, 1.0684291861,1.7050932552,-1$. $4604130951 \backslash C, 1.6708765704,0.9086801561,0.443543709 \backslash H, 2.7283113041,1.03$ 3207541,0.2576562564\|Version=ES64L-G16RevB.01 \State=1-A1\HF=-248.2545 03 $\backslash R M S D=5.451 \mathrm{e}-09 \backslash \mathrm{RMSF}=1.916 \mathrm{e}-04 \backslash \mathrm{Dipole}=0.5695,-0.2509929,0.6369 \backslash \mathrm{Quadr}$ upole $=1.7713276,-1.9866992,0.2153716,1.1807218,-2.3112908,-1.3130393 \backslash \mathrm{P}$ $\mathrm{G}=\mathrm{C} 02 \mathrm{~V}[\mathrm{C} 2(\mathrm{H} 1 \mathrm{C} 1 \mathrm{~N} 1), \mathrm{SGV}(\mathrm{C} 4 \mathrm{H} 4)] \backslash \backslash @$

PYR1.xyz-M062X-MG3S

$1 \backslash 1 \backslash G I N C-R 218 \backslash F O p t \backslash R M 062 X \backslash G e n \backslash C 5 H 6 N 1(1+) \backslash R O O T \backslash 23-J u l-2019 \backslash 0 \backslash \backslash \#$ M062X/G

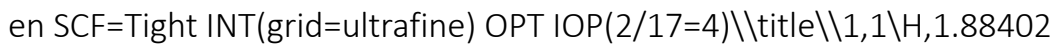

$42622,0.0769445418,2.3918313157 \backslash C, 1.1923471624,0.3817497648,1.61829022$ $32 \backslash \mathrm{C},-0.1760512541,0.2386162869,1.8170511897 \backslash \mathrm{H},-0.5750173639,-0.172821$ $3727,2.7317491567 \backslash \mathrm{C},-1.0377469092,0.6326165465,0.8202952621 \backslash \mathrm{H},-2.11280$ $78524,0.5583067591,0.8880621627 \backslash \mathrm{N},-0.5376401765,1.1441129786,-0.316451$ $1419 \backslash C, 0.777696802,1.2963573306,-0.5414800765 \backslash \mathrm{H}, 1.0621150789,1.7190840$ $517,-1.4934667843 \backslash \mathrm{C}, 1.6747944091,0.9153003087,0.4287206322 \backslash \mathrm{H}, 2.7325174$ $157,1.0364398755,0.2507472579 \backslash H,-1.1864529276,1.430028929,-1.042054641$ 6\|Version=ES64L-G16RevB.01 \State $=1-A 1 \backslash H F=-248.6181263 \backslash R M S D=5.354 \mathrm{e}-09 \backslash$ RMSF $=3.043 e-05 \backslash$ Dipole $=-0.4706858,0.2074198,-0.5263942 \backslash$ Quadrupole $=3.982$ $9253,-6.4853128,2.5023875,-0.099221,1.4430423,-4.6893145 \backslash P G=C 02 V[C 2(H$ 1C1N1H1),SGV(C4H4)]\\@

PYR2-BASE.xyz-M062X-MG3S

$1 \backslash 1 \backslash G I N C-R 222 \backslash F O p t \backslash R M 062 X \backslash G e n \backslash C 5 H 4 C l 1 N 1 \backslash R O O T \backslash 23-J u l-2019 \backslash 0 \backslash \backslash \#$ M062X/Ge n SCF=Tight INT(grid=ultrafine) OPT IOP(2/17=4)\title $\backslash 0,1 \backslash H, 1.136451$ $5977,2.8792992901,0.6070233926 \backslash C, 0.8200896601,2.0059365956,0.053074180$ $9 \backslash \mathrm{C},-0.5252370107,1.7764346439,-0.1678522272 \backslash \mathrm{H},-1.2917344394,2.4436634$ $92,0.1951576437 \backslash \mathrm{C},-0.8668830286,0.6363181398,-0.8867362376 \backslash \mathrm{Cl},-2.54944$ $75867,0.3156880405,-1.1836267284 \backslash \mathrm{N},-0.0066369426,-0.2308672551,-1.3666$ $510407 \backslash \mathrm{C}, 1.2874305104,0.004246021,-1.1453473419 \backslash \mathrm{H}, 1.9759617884,-0.7262$ $078125,-1.5517466445 \backslash \mathrm{C}, 1.7515450299,1.1039593693,-0.4439040566 \backslash \mathrm{H}, 2.811$ 2607557,1.2471529058,-0.2929065396\|Version=ES64L-G16RevB.01\State=1-A ' $\mathrm{HF}=-707.8635773 \backslash \mathrm{RMSD}=4.587 \mathrm{e}-09 \backslash \mathrm{RMSF}=8.197 \mathrm{e}-05 \backslash$ Dipole $=0.9179748,0.799$ $3587,0.5445306 \backslash$ Quadrupole $=2.4979243,-0.5287744,-1.9691499,-0.5559758,-$ $0.0243169,1.3580894 \backslash P G=C S[S G(C 5 H 4 C \mid 1 N 1)] \backslash \$

PYR2.xyz-M062X-MG3S

$1 \backslash 1 \backslash G I N C-R 228 \backslash F O p t \backslash R M 062 X \backslash G e n \backslash C 5 H 5 C l 1 N 1(1+) \backslash R O O T \backslash 23-J u l-2019 \backslash 0 \backslash \backslash \#$ M062

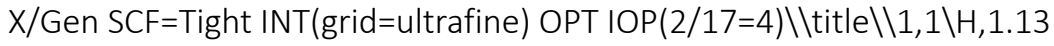
$17890314,2.8603803475,0.5951377135 \backslash C, 0.8131315151,1.9875429393,0.04137$ $92598 \backslash \mathrm{C},-0.5392586295,1.7743514786,-0.1699729485 \backslash \mathrm{H},-1.2935317799,2.452$ 0520969,0.2001791064\C,-0.9295621091,0.6548481753,-0.8791258435\Cl,-2. $5422439185,0.2736071056,-1.2089720628 \backslash \mathrm{N}, 0.0079155076,-0.191270869,-1.3$ 415193932 \C,1.3286337684,-0.005306467,-1.1487384514\Н,1.9829834599,-0. 7545702748,-1.5686986584\C,1.7611362903,1.091904868,-0.4507230136\Н,2. $8180815793,1.2432361369,-0.2949079306 \backslash H,-0.3076447257,-1.0063656468,-1$ $.8597175987 \backslash \backslash$ Version=ES64L-G16RevB.01 \State $=1-A^{\prime} \backslash H F=-708.2162453 \backslash R M S D=$ 6.920e-09\RMSF=6.891e-05\Dipole $=1.3003451,-0.2326003,-0.064864 \backslash$ Quadrup ole $=5.1397779,0.4747952,-5.6145731,-0.0311963,0.8461227,6.0185952 \backslash P G=C$ S[SG(C5H5Cl1N1)]\\@ 
PYR3-BASE.xYZ-M062X-MG3S

$1 \backslash 1 \backslash G I N C-R 246 \backslash F O p t \backslash R M 062 X \backslash G e n \backslash C 7 H 9 N 1 \backslash R O O T \backslash 23-J u l-2019 \backslash 0 \backslash \backslash \#$ M062X/Gen S

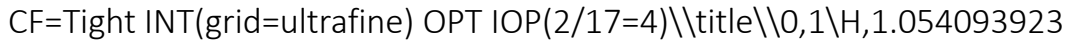
$4,0.8332220734,-2.6482753347 \backslash \mathrm{C}, 0.5773239009,0.1355834686,-1.9723156328$ $\backslash \mathrm{C},-0.7975749086,-0.0225287197,-1.9801455851 \backslash \mathrm{H},-1.4224111581,0.5438626$ $368,-2.6575607789 \backslash C,-1.3740872837,-0.9298979735,-1.095431977 \backslash C,-2.8627$ $203803,-1.1363094846,-1.0667246279 \backslash N,-0.6424208811,-1.649220541,-0.242$ $9738534 \backslash C, 0.6830794993,-1.4996094755,-0.2324475201 \backslash C, 1.4606755449,-2.3$ $336301912,0.7469462494 \backslash \mathrm{C}, 1.332895667,-0.6125409644,-1.0864100807 \backslash \mathrm{H}, 2.4$ $098055492,-0.5174309609,-1.0499402977 \backslash \mathrm{H},-3.1161976026,-1.8785283038,-0$ $.3153146404 \backslash \mathrm{H},-3.3783218684,-0.2043111916,-0.8329576193 \backslash \mathrm{H},-3.225218240$ $2,-1.476008593,-2.0374524706 \backslash \mathrm{H}, 0.7804477571,-2.9576647641,1.3193337542$ $\backslash \mathrm{H}, 2.1768967597,-2.9720696202,0.2287426272 \backslash \mathrm{H}, 2.0237931315,-1.700372218$

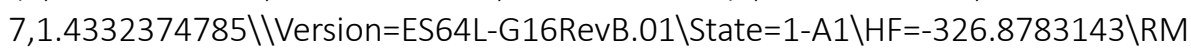
$\mathrm{SD}=4.220 \mathrm{e}-09 \backslash \mathrm{RMSF}=5.636 \mathrm{e}-05 \backslash \mathrm{Dipole}=0.2718748,0.3978236,-0.3854614 \backslash$ Quad rupole $=2.7114791,-1.5847651,-1.126714,-0.7564111,1.5250096,-1.5937351 \backslash$ PG=C02V [C2(H1C1N1),SGV(C6H4),X(H4)]\\@

PYR3.xyz-M062X-MG3S

$1 \backslash 1 \backslash G I N C-R 384 \backslash F O p t \backslash R M 062 X \backslash G e n \backslash C 7 H 10 N 1(1+) \backslash R O O T \backslash 23-J u l-2019 \backslash 0 \backslash \backslash \#$ M062X/

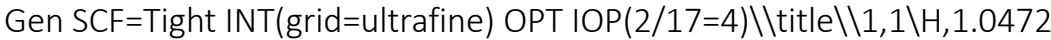
$004656,0.8224658204,-2.6376391881 \backslash C, 0.5705407706,0.1251789271,-1.96204$ $79876 \backslash \mathrm{C},-0.8089293229,-0.020013394,-1.9844069482 \backslash \mathrm{H},-1.4240184619,0.550$ $27462,-2.6649274448 \backslash C,-1.4137144085,-0.9107044245,-1.1209091664 \backslash C,-2.8$ $859342577,-1.1467022514,-1.0591945039 \backslash \mathrm{N},-0.6129949956,-1.6061694252,-0$ $.2845695736 \backslash C, 0.732326269,-1.5049575992,-0.2201186525 \backslash C, 1.4646020172$,$2.3513951635,0.7669227027 \backslash \mathrm{C}, 1.3439795081,-0.6161684068,-1.080733559 \backslash \mathrm{H}$, $2.418860665,-0.5138445951,-1.0518969462 \backslash \mathrm{H},-1.0601480169,-2.2602921554$, $0.3492004926 \backslash \mathrm{H},-3.1499165508,-1.889002161,-0.3089881337 \backslash \mathrm{H},-3.400456295$ 4,-0.2160626807,-0.8228477929\Н,-3.24694103,-1.4889713242,-2.028322306 $6 \backslash \mathrm{H}, 0.7912196867,-2.9803294344,1.3452853263 \backslash \mathrm{H}, 2.1779613087,-2.99116346$ $39,0.2487550187 \backslash \mathrm{H}, 2.0244460433,-1.7182548204,1.4542295324 \backslash \backslash V e r s i o n=E S 6$ 4L-G16RevB.01 \State=1-A1 \HF=-327.2555546\RMSD=7.100e-09 \RMSF=4.334e-05 |Dipole $=-0.1809002,-0.2646319,0.256398 \backslash$ Quadrupole $=5.8076952,-3.3892546$ $,-2.4184406,0.0124618,1.9586884,-6.6370314 \backslash P G=C 02 V$ [C2(H1C1N1H1),SGV(C 6H4),X(H4)]\\@

PYR4-BASE.xyz-M062X-MG3S

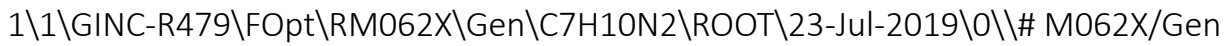
SCF=Tight INT(grid=ultrafine) OPT IOP(2/17=4)\|title \0,1\N,1.22279917 $88,-1.8603971976,-1.7832100323 \backslash C, 0.5628643305,-1.1210364436,-0.8444898$ $441 \backslash \mathrm{C},-0.8363545384,-0.9850273342,-0.8531571053 \backslash \mathrm{H},-1.4454426819,-1.461$ $6837084,-1.6051608186 \backslash \mathrm{C},-1.4466699289,-0.222443235,0.1250321755 \backslash \mathrm{H},-2.5$ $262364651,-0.119452602,0.1156261676 \backslash \mathrm{N},-0.8072301973,0.4139546633,1.104$ $3925287 \backslash C, 0.5177875253,0.2822624059,1.1085567661 \backslash H, 1.0450286846,0.7980$ $718078,1.9036145441 \backslash \mathrm{C}, 1.2425533194,-0.4509172569,0.1876681848 \backslash \mathrm{H}, 2.3165$ $752328,-0.4951513671,0.2783297713 \backslash C, 0.4767276964,-2.5306146038,-2.8249$ $262744 \backslash \mathrm{C}, 2.6637769842,-1.9687209827,-1.7299589332 \backslash \mathrm{H},-0.2301698877,-3.2$ $53834835,-2.4096367598 \backslash \mathrm{H}, 1.1668767979,-3.0638042903,-3.4714791539 \backslash \mathrm{H},-0$ $.0843743598,-1.8194017278,-3.4369375533 \backslash H, 2.9970626366,-2.4246988025,-$ $0.7938916387 \backslash \mathrm{H}, 3.1428581645,-0.9902656953,-1.8211924323 \backslash H, 3.0053796828$ ,-2.5914587199,-2.5510149344||Version=ES64L-G16RevB.01\State=1-A1 \HF=$382.2120038 \backslash \mathrm{RMSD}=6.059 \mathrm{e}-09 \backslash \mathrm{RMSF}=4.021 \mathrm{e}-05 \backslash$ Dipole $=0.8974209,-1.0054292$, $-1.2765307 \backslash$ Quadrupole=3.1387234,-2.0568106,-1.0819127,1.4962238,2.8671 


\section{1,0.6115162\PG=C02V [C2(N1C1N1),SGV(C6H6),X(H4)]\\@}

PYR4.xyz-M062X-MG3S

$1 \backslash 1 \backslash G I N C-R 480 \backslash F O p t \backslash R M 062 X \backslash G e n \backslash C 7 H 11 N 2(1+) \backslash R O O T \backslash 23-J u l-2019 \backslash 0 \backslash \backslash \#$ M062X/

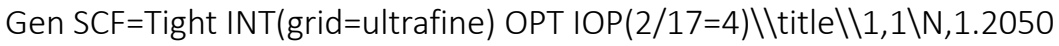

417088,-1.8403718932,-1.7579834161\C,0.5631970973,-1.1213653222,-0.845 $0062472 \backslash \mathrm{C},-0.8574990463,-0.9865080419,-0.8582772704 \backslash \mathrm{H},-1.460550172,-1$. 4647960309,-1.6117506025\C,-1.4811713602,-0.2435594099,0.090712237\H,$2.5529792291,-0.1163425256,0.1162778251 \backslash \mathrm{N},-0.77717638,0.3801466022,1.0$ $615773098 \backslash \mathrm{C}, 0.5699304308,0.2834264803,1.1176626871 \backslash \mathrm{H}, 1.0570592891,0.81$ $1178171,1.923760368 \backslash \mathrm{C}, 1.2579372083,-0.4429928139,0.2008843096 \backslash \mathrm{H}, 2.3305$ $781263,-0.4907482632,0.2864003957 \backslash \mathrm{H},-1.265430432,0.9270981001,1.756083$ $1046 \backslash C, 0.4635294836,-2.5232985918,-2.8168704418 \backslash C, 2.6616717871,-1.9585$ $338476,-1.7162994035 \backslash \mathrm{H},-0.2325194149,-3.248385576,-2.3947839004 \backslash H, 1.16$ $5093193,-3.0499369362,-3.4527421716 \backslash \mathrm{H},-0.0860079425,-1.806523241,-3.42$ $73079618 \backslash \mathrm{H}, 2.9848611484,-2.4217497808,-0.7838982624 \backslash \mathrm{H}, 3.1313726208,-0$. $9798874458,-1.8164223237 \backslash \mathrm{H}, 2.9867083011,-2.5819126463,-2.5406916515 \backslash \backslash \mathrm{V}$ ersion=ES64L-G16RevB.01 \State=1-A1 \HF=-382.6048615 $\backslash$ RMSD $=7.523 \mathrm{e}-09 \backslash \mathrm{RMSF}$ $=8.367 \mathrm{e}-05 \backslash$ Dipole $=-0.4414597,0.4945316,0.6279443 \backslash$ Quadrupole $=2.4855111$, $-4.6892637,2.2037526,-5.0471173,-4.7503875,11.8763153 \backslash P G=C 02 V$ [C2(N1C1 $\mathrm{N} 1 \mathrm{H} 1), \mathrm{SGV}(\mathrm{C} 6 \mathrm{H} 6), \mathrm{X}(\mathrm{H} 4)] \backslash \backslash @$

PYR5-BASE.xyz-M062X-MG3S

$1 \backslash 1 \backslash G I N C-R 523 \backslash F O p t \backslash R M 062 X \backslash G e n \backslash C 8 H 11 N 1 \backslash R O O T \backslash 23-J u l-2019 \backslash 0 \backslash \backslash \#$ M062X/Gen

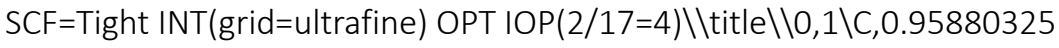
$09,-1.876194883,2.2738257229 \backslash C, 0.0297831047,-0.8372289012,1.7123904112$ \C,-1.3486277639,-0.9830303314,1.8142807688\Н,-1.7734233696,-1.8522607 $263,2.302400095 \backslash C,-2.1866730194,-0.0086360871,1.287229752 \backslash C,-3.6807491$ $622,-0.1457680193,1.3860876519 \backslash \mathrm{N},-1.7144756631,1.0809662541,0.67628302$ $58 \backslash C,-0.3954078167,1.2352664276,0.5705924199 \backslash C, 0.0984152589,2.47408130$ $88,-0.1233597001 \backslash C, 0.5038366149,0.2993763366,1.0753723465 \backslash H, 1.56891707$ $31,0.4642914809,0.9676893283 \backslash H, 2.0001108851,-1.607482075,2.1089954909 \backslash$ $\mathrm{H}, 0.776830743,-2.845423261,1.8090583191 \backslash \mathrm{H}, 0.8003644179,-1.9938428465,3$ $.3460115626 \backslash \mathrm{H},-4.1595827324,0.7101144727,0.9192002845 \backslash \mathrm{H},-3.9964936539$, $-0.2036522623,2.4281988017 \backslash \mathrm{H},-4.0200420869,-1.0557667079,0.8902817258 \backslash$ $H,-0.7466092458,3.0764082779,-0.4441523593 \backslash H, 0.7011765685,2.2157283451$ $,-0.9946238326 \backslash \mathrm{H}, 0.7247244371,3.0678223688,0.5432563856 \backslash \backslash$ Version=ES64L

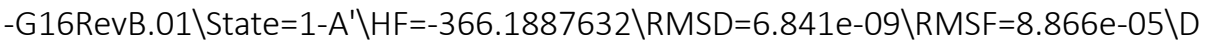
ipole $=0.4937809,-0.5601285,0.30279 \backslash$ Quadrupole $=1.6500306,0.0882123,-1.7$ 382429,1.7659571,-1.0434185,-1.5120756\PG=CS [SG(C8H5N1),X(H6)]\\@

PYR5.xyz-M062X-MG3S

$1 \backslash 1 \backslash G I N C-R 649 \backslash F O p t \backslash R M 062 X \backslash G e n \backslash C 8 H 12 N 1(1+) \backslash R O O T \backslash 23-J u l-2019 \backslash 0 \backslash \backslash \#$ M062X/ Gen SCF=Tight INT(grid=ultrafine) OPT IOP(2/17=4)\title \1,1\C,0.9521 94432,-1.8647037557,2.2682445777\\,0.0294847451,-0.8306456717,1.709139 $4631 \backslash \mathrm{C},-1.3540631017,-0.9900080908,1.8185412671 \backslash \mathrm{H},-1.7694187142,-1.860$ $8554841,2.3075598139 \backslash \mathrm{C},-2.2137283849,-0.0462440667,1.3085241889 \backslash \mathrm{C},-3.7$ $02087345,-0.1432268052,1.3849464061 \backslash \mathrm{N},-1.6705352848,1.0335677809,0.701$ $7340262 \backslash C,-0.3463902759,1.25305791,0.5599093207 \backslash C, 0.0980331235,2.49677$ $0876,-0.1362237005 \backslash C, 0.5167098802,0.3081800189,1.070491482 \backslash H, 1.5808267$ $768,0.4677880727,0.9658182364 \backslash \mathrm{H},-2.3048434209,1.7292625931,0.325784628$ $6 \backslash H, 1.994455501,-1.6048864044,2.1083602085 \backslash H, 0.7544986646,-2.831050241$ $5,1.8033305534 \backslash \mathrm{H}, 0.7778774309,-1.9801656583,3.3384484504 \backslash \mathrm{H},-4.19072197$ $82,0.7100688522,0.9194235649 \backslash \mathrm{H},-4.0178189802,-0.1970817685,2.425913343$ 
$8 \backslash \mathrm{H},-4.0412619712,-1.0503038475,0.8865782689 \backslash \mathrm{H},-0.7431751518,3.1054745$ $346,-0.4608047299 \backslash \mathrm{H}, 0.696899511,2.2394750882,-1.0089001814 \backslash \mathrm{H}, 0.7203392$ 9,3.0925802656,0.5302239861 \|Version=ES64L-G16RevB.01\State=1-A'\HF=-3 66.5719205\RMSD=9.095e-09 \RMSF $=5.594$ e-05\Dipole $=-0.4514043,0.4766819$,$0.2573405 \backslash Q u a d r u p o l e=4.9884611,1.8852513,-6.8737125,-0.7097397,0.15376$ 89,-6.9904583\PG=CS [SG(C8H6N1),X(H6)]\\@

T1-BASE.xyz-M062X-MG3S

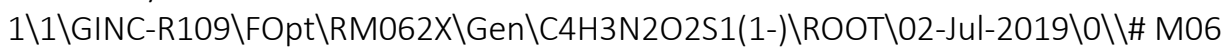
2X/Gen SCF=Tight INT(grid=ultrafine) OPT IOP(2/17=4)\\itle \-1,1\S,-2 $.6702852916,-0.4275535714,-0.0001490524 \backslash N, 1.2881383161,-1.2894128475,-$ $0.0002659679 \backslash C,-0.1100602546,-1.3813038377,-0.0001765621 \backslash C,-0.94796096$ $63,-0.3155211142,-0.0001382041 \backslash C,-0.2758861389,1.011175576,-0.00021250$ $59 \backslash \mathrm{N}, 1.1358473973,0.9704839304,-0.0001236909 \backslash \mathrm{H},-0.4968408513,-2.388788$ $6401,-0.0002908742 \backslash C, 1.9706188029,-0.1193804501,0.0001387555 \backslash 0,-0.8013$ 207474,2.0987245523,-0.0001167093\0,3.1929110019,-0.0305991817,0.00046 $15871 \backslash H, 1.5923171906,1.8700423271,-0.0000051121 \backslash H, 1.8533835413,-2.1198$ $517431,0.0004683363 \backslash \mid$ Version=ES64L-G16RevB.01\State=1-A \HF=-812.461669 $2 \backslash$ RMSD $=3.205 e-09 \backslash R M S F=3.730$ e-05\Dipole $=2.5339738,-0.8059957,0.0001436 \backslash$ Quadrupole $=-21.1518547,10.4295619,10.7222928,-1.1190059,-0.0016835,-0$. 0015335\PG=C01 [X(C4H3N2O2S1)]\\@

T1.xyz-M062X-MG3S

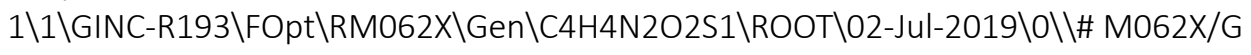
en SCF=Tight INT(grid=ultrafine) OPT IOP(2/17=4)\title \\0,1\S,2.60086 $71468,-0.3333544503,-0.0000361426 \backslash \mathrm{N},-1.3368282354,-1.2846961149,0.0000$ $734629 \backslash C, 0.0350566021,-1.4072010463,-0.0000053429 \backslash C, 0.8443281983,-0.33$ $87480472,-0.0000603546 \backslash \mathrm{C}, 0.2443781229,1.0007563688,0.0001547333 \backslash \mathrm{N},-1.1$ $478937972,1.0063642248,0.0001186588 \backslash \mathrm{H}, 0.4056704497,-2.4222089812,0.000$ $0993372 \backslash \mathrm{C},-2.0022155264,-0.0779772376,-0.0004930446 \backslash 0,0.8754596217,2.0$ $286374923,0.0001686817 \backslash 0,-3.2037975718,0.0174277508,-0.0000387307 \backslash \mathrm{H},-1$ $.5924125062,1.9144041537,0.0002429521 \backslash \mathrm{H},-1.9279223222,-2.0996083036,-0$ $.0002370772 \backslash \mathrm{H}, 2.7248148178,-1.6651568093,-0.0000941334 \backslash \backslash V e r s i o n=E S 64 L-$

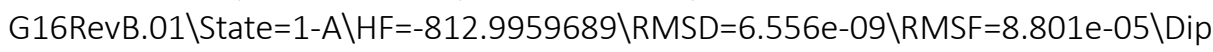
ole $=0.1523879,-1.8227623,-0.0004383 \backslash$ Quadrupole $=-7.3379204,6.9651196,0$. 3728008,-3.6973443,0.0013242,0.0007698\PG=C01 [X(C4H4N2O2S1)]\\@

T2-BASE.xyz-M062X-MG3S

$1 \backslash 1 \backslash G I N C-R 365 \backslash F O p t \backslash R M 062 X \backslash G e n \backslash C 8 H 701 S 1(1-) \backslash R O O T \backslash 02-J u l-2019 \backslash 0 \backslash \backslash \#$ M062X /Gen SCF=Tight INT(grid=ultrafine) OPT IOP(2/17=4)\|title \-1,1\S,-3.5 031568106,-0.0826257446,-0.0003405415\C,1.0703243292,0.0834139367,0.00 $01242292 \backslash C, 0.3804158931,-1.1408984841,-0.0001922892 \backslash C,-0.9939208466,-1$ $.1936367583,-0.0003468169 \backslash C,-1.7955337479,-0.0208349236,-0.0001631441 \backslash$ C,- $-1.0777248865,1.208583586,0.0001468824 \backslash \mathrm{H}, 0.8124737463,2.2066777272,0$ $.0005045114 \backslash \mathrm{H},-1.6557037161,2.1235114072,0.0002885258 \backslash \mathrm{C}, 0.2937472897,1$ $.2560121211,0.0002731938 \backslash \mathrm{H},-1.5023686047,-2.1487738003,-0.0006103081 \backslash \mathrm{H}$ $, 0.9348322948,-2.0721878463,-0.000339885 \backslash C, 2.5272574746,0.1733448152,0$ $.0002389525 \backslash 0,3.1333230047,1.2348372291,0.0001451847 \backslash \mathrm{C}, 3.3288446971,-1$ $.12220492,0.0001529384 \backslash \mathrm{H}, 3.094144026,-1.7226660682,-0.8792390381 \backslash \mathrm{H}, 4.3$ $858315271,-0.8713367464,0.0005318796 \backslash \mathrm{H}, 3.0935843296,-1.7232975306,0.87$ $89437252 \backslash \backslash$ Version=ES64L-G16RevB.01 \State $=1-A \backslash H F=-782.5165142 \backslash$ RMSD $=9.20$ 3e-09 \RMSF=3.787e-05\Dipole $=2.6410108,-0.9016324,0.0002503 \backslash$ Quadrupole $=$ $-25.388175,12.7669402,12.6212347,-7.3893565,-0.0029427,0.0003448 \backslash P G=C 0$ $1[\mathrm{X}(\mathrm{C} 8 \mathrm{H} 7 \mathrm{O} 1 \mathrm{~S} 1)] \backslash \backslash @$ 
T2.xyz-M062X-MG3S

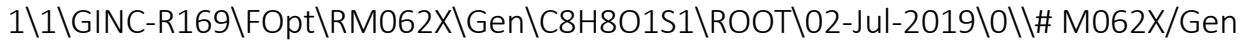
SCF=Tight INT(grid=ultrafine) OPT IOP(2/17=4)\title \\0,1\S,-3.452931

9295,-0.1538335104,-0.0000048714\C,1.0937561935,0.071510718,-0.0000685 $48 \backslash C, 0.4246677574,-1.1494403937,-0.0000602079 \backslash C,-0.9590887433,-1.19876$ $00202,-0.0000397495 \backslash C,-1.6988502077,-0.0185379029,-0.0000279199 \backslash C,-1.0$ $346390117,1.2089069449,-0.0000361475 \backslash H, 0.8721720526,2.1922118863,-0.00$ $00612322 \backslash \mathrm{H},-1.6004997937,2.1308038764,-0.0000286225 \backslash \mathrm{C}, 0.3454764875,1.2$

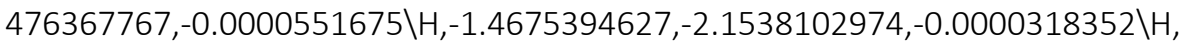
$0.9797929519,-2.0775563496,-0.0000701809 \backslash C, 2.5854110482,0.1721344413,-$ $0.0000913803 \backslash 0,3.1291856852,1.2506838444,-0.0000876237 \backslash C, 3.3924418247$, $-1.1047668802,-0.0000799976 \backslash \mathrm{H}, 3.1583524816,-1.7048847384,-0.8799966164$ $\backslash \mathrm{H}, 4.447500275,-0.8509119426,-0.0000627641 \backslash \mathrm{H}, 3.1583244111,-1.704904107$ $5,0.8798144073 \backslash \mathrm{H},-3.7120970202,1.156440655,0.0000114573 \backslash \backslash$ Version=ES64L - G16RevB.01 \State $=1-A \backslash H F=-783.0465179 \backslash R M S D=8.797 \mathrm{e}-09 \backslash \mathrm{RMSF}=3.932 \mathrm{e}-05 \backslash \mathrm{Di}$ pole $=-0.564676,-0.6649819,0.0000079 \backslash$ Quadrupole $=-2.1041887,4.0822202,-1$ $.9780315,-9.3219594,-0.0000381,0.0001342 \backslash P G=C 01[X(C 8 H 801 S 1)] \backslash \backslash @$

T3-BASE.xyz-M062X-MG3S

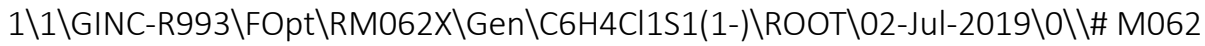
X/Gen SCF=Tight INT(grid=ultrafine) OPT IOP(2/17=4)\\title \-1,1\S,2.7 707405795,-0.9062317281,-0.0004376099\C,-1.2013592549,1.3945100008,0.0 $001229237 \backslash C,-1.1508818745,0.0087065269,0.0000640736 \backslash C, 0.0287871105,-0$. $7043032328,0.0002669231 \backslash C, 1.2793247838,-0.0408402374,0.0009378773 \backslash C, 1$. $2209236056,1.3734634946,0.0000845976 \backslash \mathrm{H}, 0.0307532841,3.1457690095,-0.00$ $03977051 \backslash H, 2.1581470861,1.914006624,-0.0002637945 \backslash C, 0.0224981432,2.061$ $6563036,-0.0000679385 \backslash H, 0.0108592178,-1.7847753184,0.0000907214 \backslash \mathrm{H},-2.1$ 462404341,1.916709109,-0.0000407293\Cl,-2.6735342472,-0.8779695516,-0. $0001773395 \backslash \mid$ Version=ES64L-G16RevB.01\State $=1-A \backslash H F=-1089.4767869 \backslash$ RMSD $=5$ $.687 \mathrm{e}-09 \backslash \mathrm{RMSF}=5.639 \mathrm{e}-05 \backslash$ Dipole $=-2.5563588,1.1963352,0.0000524 \backslash \mathrm{Quadrupo}$ $l e=-14.8988679,7.5322498,7.3666181,4.885164,0.0003463,-0.0012193 \backslash P G=C 0$ $1[\mathrm{X}(\mathrm{C} 6 \mathrm{H} 4 \mathrm{Cl} 1 \mathrm{~S} 1)] \backslash \backslash @$

T3.xyz-M062X-MG3S

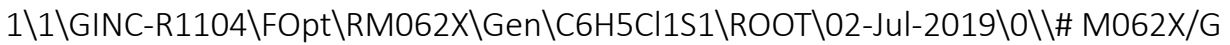
en SCF=Tight INT(grid=ultrafine) OPT IOP(2/17=4)\title \} \backslash 0 , 1 \backslash S , 2 . 6 8 6 0 8 $79494,-0.9356244908,-0.0000023356 \backslash C,-1.2329251197,1.3859014718,-0.0000$ $023356 \backslash C,-1.1990619762,0.0006173021,-0.0000023356 \backslash C,-0.0042971989,-0.6$ $978719675,-0.0000023356 \backslash C, 1.1963520833,0.0071979959,-0.0000023356 \backslash C, 1$. $1804686284,1.3991661583,-0.0000023356 \backslash \mathrm{H},-0.0350302867,3.1565973134,-0$. $0000023356 \backslash H, 2.1087153292,1.9535810512,-0.0000023356 \backslash C,-0.0294989129,2$ $.0752208295,-0.0000023356 \backslash \mathrm{H},-0.013358698,-1.7790691432,-0.0000023356 \backslash \mathrm{H}$ ,-2.1799131692,1.9044386825,-0.0000023356 \Cl,-2.6886001255,-0.88711328 $65,-0.0000023356 \backslash \mathrm{H}, 3.5258004968,0.1033620833,-0.0000023356 \backslash \backslash V e r s i o n=E S$

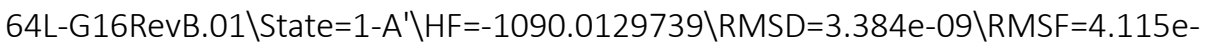
05\Dipole $=0.4998751,0.7256829,0 . \backslash$ Quadrupole $=1.1438933,2.9095258,-4.053$ 4192,1.1195089,0.,0.|PG=CS [SG(C6H5Cl1S1)]\\@

T4-BASE.xyz-M062X-MG3S

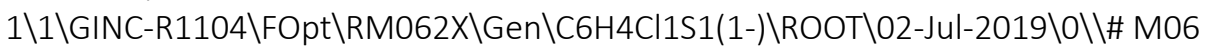
2X/Gen SCF=Tight INT(grid=ultrafine) OPT IOP(2/17=4)\\title \-1,1\S,3. 2094053636,-0.0000001648,0.0000788176\C,-1.3529947072,-0.0000002616,-0 $.000056739 \backslash C,-0.66039213,-1.2009815168,-0.0000329773 \backslash C, 0.7242527976,-1$ 
$.1937894009,-0.000064448 \backslash C, 1.4820606153,-0.0000002014,-0.0002672505 \backslash C$, $0.7242527469,1.1937889659,-0.000064448 \backslash \mathrm{H},-1.2066089068,2.1355488862,0$. $0000239869 \backslash \mathrm{H}, 1.2615489652,2.1331477585,0.0000053348 \backslash \mathrm{C},-0.6603921809,1$. $200981023,-0.0000329773 \backslash \mathrm{H}, 1.2615490558,-2.1331481707,0.0000053348 \backslash \mathrm{H},-1$ .2066088161,-2.1355494032,0.0000239869 \Cl,-3.1084367237,-0.0000002988, $0.000015379 \backslash \mid$ Version=ES64L-G16RevB.01 \State $=1-A^{\prime} \backslash H F=-1089.4755196 \backslash$ RMSD $=5.293 \mathrm{e}-09 \backslash \mathrm{RMSF}=2.948 \mathrm{e}-05 \backslash$ Dipole $=-3.048304,0 .,-0.0000141 \backslash$ Quadrupole $=-2$ $2.4503684,13.1904895,9.2598789,-0.0000008,-0.0002017,0 . \mid P G=C S[S G(C 2 C l$ 1S1),X(C4H4)]\\@

T4.xyz-M062X-MG3S

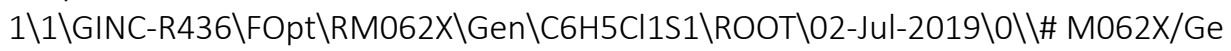
n SCF=Tight INT(grid=ultrafine) OPT IOP(2/17=4)\title \\0,1\S,3.146564 $1518,-0.0812417653,0.000221097 \backslash C,-1.3913031227,0.0017100851,-0.0000288$ $264 \backslash C,-0.7046335052,1.2045098085,0.0000130347 \backslash C, 0.6813961086,1.2021023$ $158,0.0000773174 \backslash C, 1.3847067609,0.0014503218,0.0000411334 \backslash C, 0.67939999$ $19,-1.1995452415,-0.0000219505 \backslash \mathrm{H},-1.2531860293,-2.1338178701,-0.000083$ 7506\H,1.2143516751,-2.1401243239,-0.0000876819\C,-0.7056074756,-1.202 $3825803,-0.0000331236 \backslash \mathrm{H}, 1.2119137725,2.1446611375,0.0001770035 \backslash \mathrm{H},-1.25$ $14674944,2.1364738698,0.0000252394 \backslash \mathrm{Cl},-3.1251077112,0.0027731118,-0.00$ $00828554 \backslash \mathrm{H}, 3.3656458774,1.236036131,-0.0011496369|| V e r s i o n=E S 64 L-G 16 R e$ $v B .01 \backslash S t a t e=1-A \backslash H F=-1090.0126907 \backslash R M S D=2.404 e-09 \backslash R M S F=4.523 e-05 \backslash D i p o l e=$ $0.4098554,0.2927995,-0.0002983 \backslash$ Quadrupole $=-2.4763284,5.7874194,-3.3110$ $911,2.3714452,-0.0024237,-0.0018446 \backslash P G=C 01$ [X(C6H5Cl1S1)]\\@

T5-BASE.xyz-M062X-MG3S

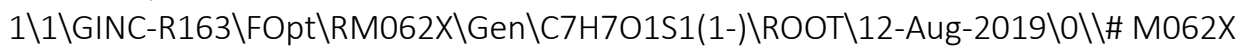
/Gen SCF=Tight INT(grid=ultrafine) OPT IOP(2/17=4)\title\\-1,1\S,-2.3 $593629005,-0.1343845239,2.1127361258 \backslash C,-1.1820525076,0.1197224581,0.85$ $94413554 \backslash \mathrm{C},-1.5473226817,0.4010702017,-0.4768089616 \backslash \mathrm{H},-2.603132588,0.4$ $56521634,-0.7098710297 \backslash \mathrm{C},-0.6174036862,0.6044418267,-1.479489427 \backslash \mathrm{H},-0$. $9303178457,0.8183928491,-2.4945591297 \backslash C, 0.7448992476,0.5384563899,-1.2$ $054155026 \backslash 0,1.6025347332,0.7553593939,-2.2706774403 \backslash C, 1.1516201772,0.2$ $646583926,0.0933989659 \backslash H, 2.2011189227,0.204499082,0.3492035701 \backslash C, 0.202$ $9708922,0.0620920826,1.0927993233 \backslash \mathrm{H}, 0.5371248311,-0.1504254014,2.10034$ $89635 \backslash C, 2.9698184428,0.6890350743,-1.9951338657 \backslash H, 3.4898219299,0.88127$ $77918,-2.9318872595 \backslash H, 3.2598053655,-0.298357446,-1.6194202294 \backslash H, 3.2709$ 843879,1.4399534476,-1.2564743967\\Version=ES64L-G16RevB.01 \State=1-A' $\backslash \mathrm{HF}=-744.3771975 \backslash \mathrm{RMSD}=3.842 \mathrm{e}-09 \backslash \mathrm{RMSF}=1.469 \mathrm{e}-04 \backslash \mathrm{Dipole}=3.2763344,0.5235$ 997,-2.6086683\Quadrupole=-1.2522613,7.5841493,-6.3318881,-3.1048915,1 5.1611296,2.9316958\PG=CS [SG(C7H5O1S1),X(H2)]\\@

T5.xyz-M062X-MG3S

$1 \backslash 1 \backslash G I N C-R 130 \backslash F O p t \backslash R M 062 X \backslash G e n \backslash C 7 H 801 S 1 \backslash R O O T \backslash 12-A u g-2019 \backslash 0 \backslash \backslash \#$ M062X/Gen SCF=Tight INT(grid=ultrafine) OPT IOP(2/17=4)\\title \0,1\S,-2.285643 $5925,-0.1325648936,2.1605381837 \backslash \mathrm{H},-2.7189130872,1.1274746119,2.2869030$ $587 \backslash \mathrm{C},-1.1103233704,0.167132771,0.8579243773 \backslash \mathrm{C},-1.5324974326,0.4306510$ 487,-0.4464897919 $\mathrm{H},-2.5909290456,0.4749507627,-0.6642525786 \backslash \mathrm{C},-0.6133$ $490975,0.6298029324,-1.4547971712 \backslash \mathrm{H},-0.92669816,0.8407866233,-2.467701$ $2322 \backslash C, 0.75381622,0.5470069778,-1.1843389859 \backslash 0,1.578853481,0.747640340$ $7,-2.2385082795 \backslash C, 1.1854616119,0.2682430098,0.1071631334 \backslash H, 2.236392150$ $7,0.1963298063,0.3421314035 \backslash \mathrm{C}, 0.2478032088,0.0861524427,1.1190991515 \backslash \mathrm{H}$ ,0.5832010297,-0.1187906003,2.1265139448\C,2.9682646697,0.6678780722,2.0126963207\} \mathrm { H } , 3 . 4 4 3 2 8 5 1 9 8 9 , 0 . 8 5 5 8 2 0 7 2 8 3 , - 2 . 9 7 0 4 4 8 1 3 4 2 \backslash H , 3 . 2 5 2 8 8 3 7 1 7 7 \text { , } 
$-0.3238986986,-1.6542563804 \backslash \mathrm{H}, 3.2942124975,1.4206840645,-1.2914943782 \backslash$ Version=ES64L-G16RevB.01 \State=1-A \HF=-744.9264761 \RMSD=3.496e-09 \RMS $\mathrm{F}=1.392 \mathrm{e}-05 \backslash$ Dipole $=0.8409809,0.3257929,-0.2899714 \backslash$ Quadrupole $=5.3321526$ ,-2.9027089,-2.4294437,-2.2547484,0.1493007,1.1489454\PG=C01 [X(C7H8O1 S1)]\\@ 